\title{
Straightforward Synthesis of Fluorinated Enals via Photocatalytic $\alpha$-Perfluoroalkenylation of Aldehydes
}

\author{
Christian Wulkesch and Constantin Czekelius* \\ Department of Organic Chemistry and Macromolecular Chemistry, Heinrich-Heine-Universität Düsseldorf, \\ 40225 Düsseldorf, Germany \\ *Correspondence: constantin.czekelius@hhu.de; Tel.: +49-211-8115362 \\ Supporting Information
}

\section{Table of content:}

$\begin{array}{ll}\text { Reaction setup and photoreactor } & \text { S1 }\end{array}$

${ }^{1} \mathrm{H}-\mathrm{NMR}-$ spectra of products of organocatalyst $\mathbf{1 b}$ synthesis $\quad$ S2 - S4

${ }^{1} \mathrm{H}-,{ }^{13} \mathrm{C}-$, and ${ }^{19} \mathrm{~F}-\mathrm{NMR}$ spectra of new enal-products $\mathbf{3 a}-\mathbf{3 o} \quad$ S5 - S53

${ }^{1} \mathrm{H}-,{ }^{13} \mathrm{C}-$, and ${ }^{19} \mathrm{~F}-\mathrm{NMR}$ spectra of new hydrazone derivatives $\mathbf{4 a}-\mathbf{4 d}, \mathbf{4 k}, 4 \mathbf{S} \quad \mathrm{S} 54-\mathrm{S} 71$

${ }^{1} \mathrm{H}-,{ }^{13} \mathrm{C}-$, and ${ }^{19} \mathrm{~F}-\mathrm{NMR}$ spectra of new allylic alcohols $\mathbf{5 a}-\mathbf{5 d} \quad \mathrm{S} 72-\mathrm{S} 83$

${ }^{1} \mathrm{H}-,{ }^{13} \mathrm{C}-$, and ${ }^{19} \mathrm{~F}-\mathrm{NMR}$ spectra of new 4-methoxybenzoate derivatives $\mathbf{6 a}, \mathbf{6 d} \quad \mathrm{S} 84-\mathrm{S} 89$ 


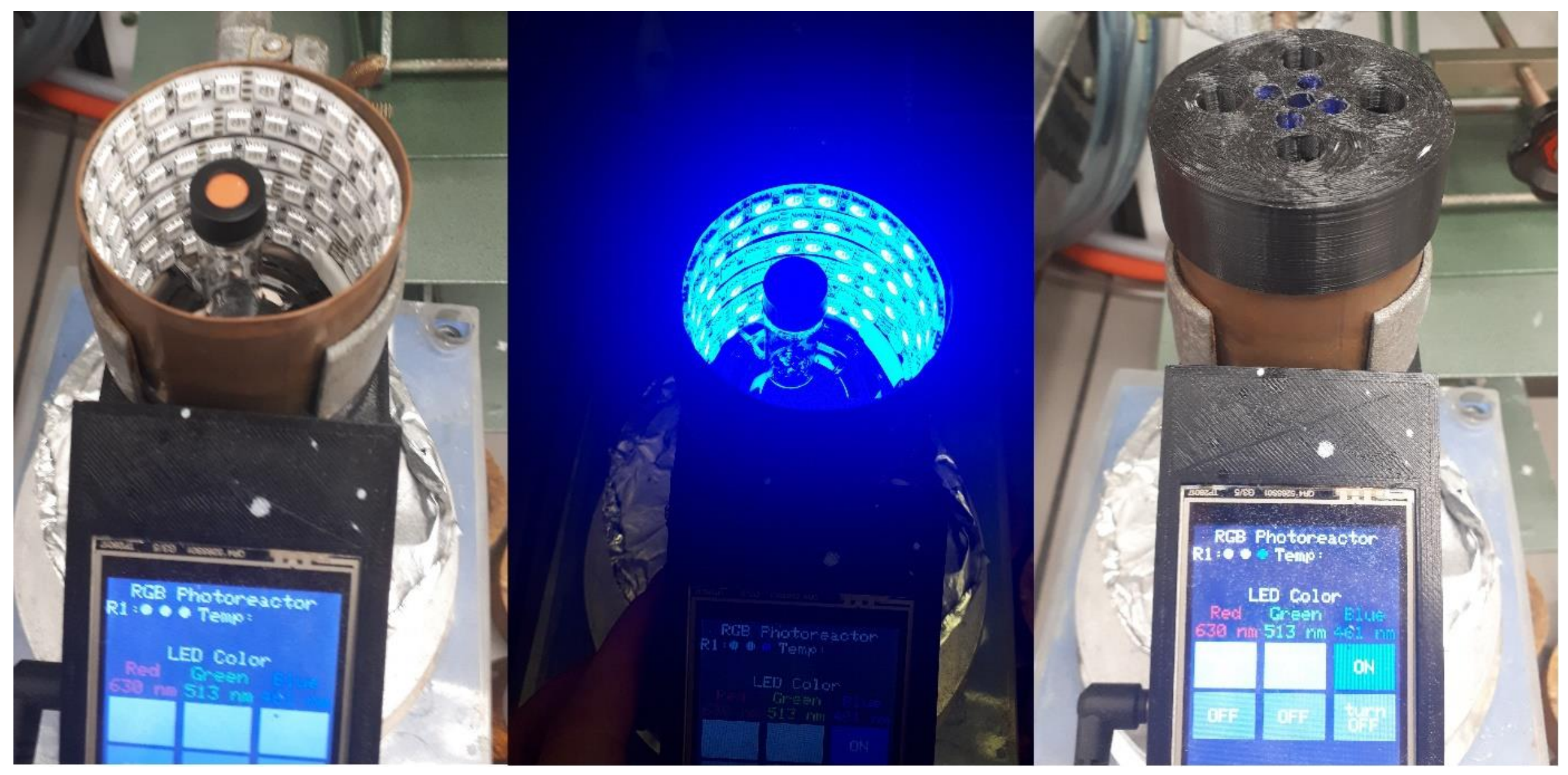

Reaction setup for irradiation of reaction mixtures in a self-built photoreactor. The irradiation is performed by a high density RGB LED strip (120 LEDs/m, $12 \mathrm{~V}$ ), which was purchased from aliexpress.com (Brand Name: XUNATA, Size: $L 120 \mathrm{~cm} \times W 1 \mathrm{~cm} \times \mathrm{T} 0.2 \mathrm{~cm}$, Protection Rate: notwaterproof). The emission maximum is at $461 \mathrm{~nm}$. The reaction vessels are made of borosilicate glass and are placed in a distance of $1-2 \mathrm{~cm}$ from the LEDs. For further information and construction using a 3D printer see: Helmecke, L.; Spittler, M.; Baumgarten, K.; Czekelius, Org. Lett. 2019, 21 (19), 7823-7827. 
1H NMR, CD3OD, $300 \mathrm{MHz}$

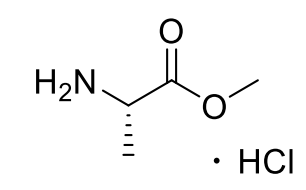

1b-1
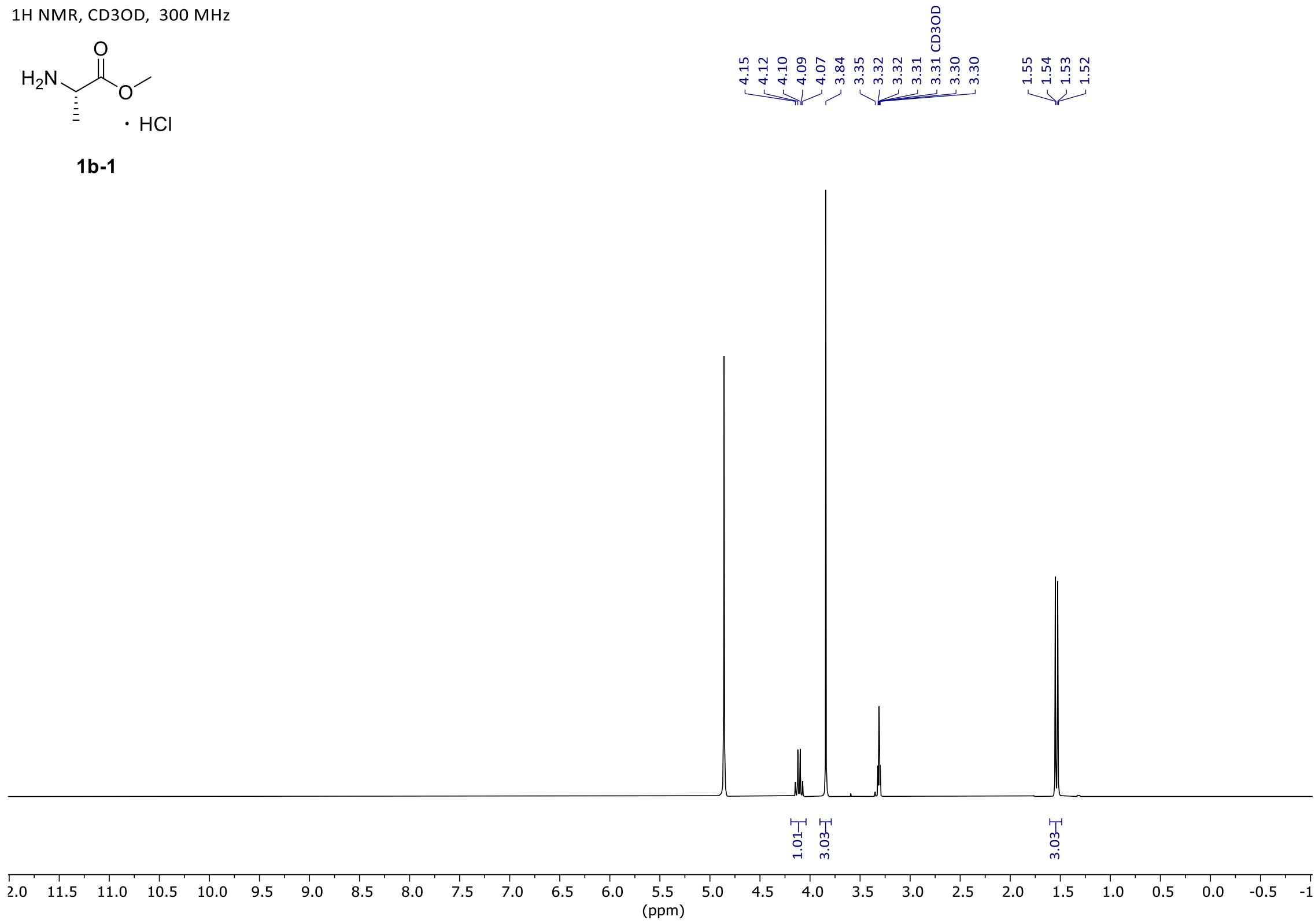
1H NMR, CD3OD, $300 \mathrm{MHz}$

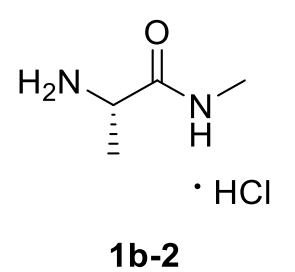

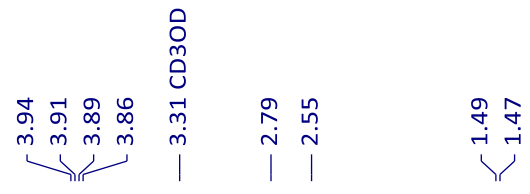

MeNH2 (s)

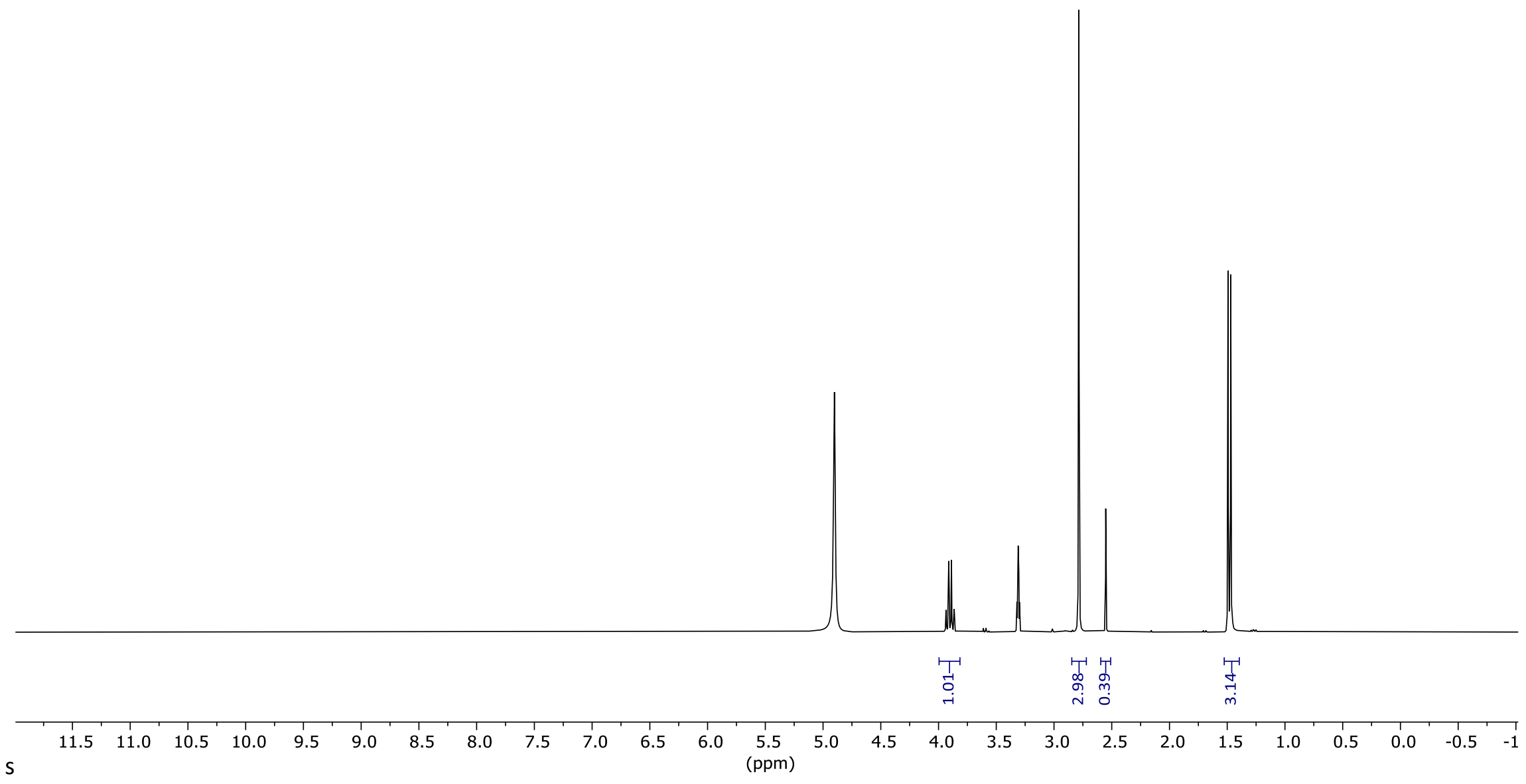


1H NMR, $\mathrm{CDCl} 3,600 \mathrm{MHz}$

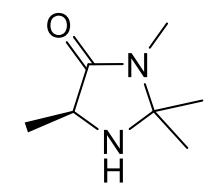

1b
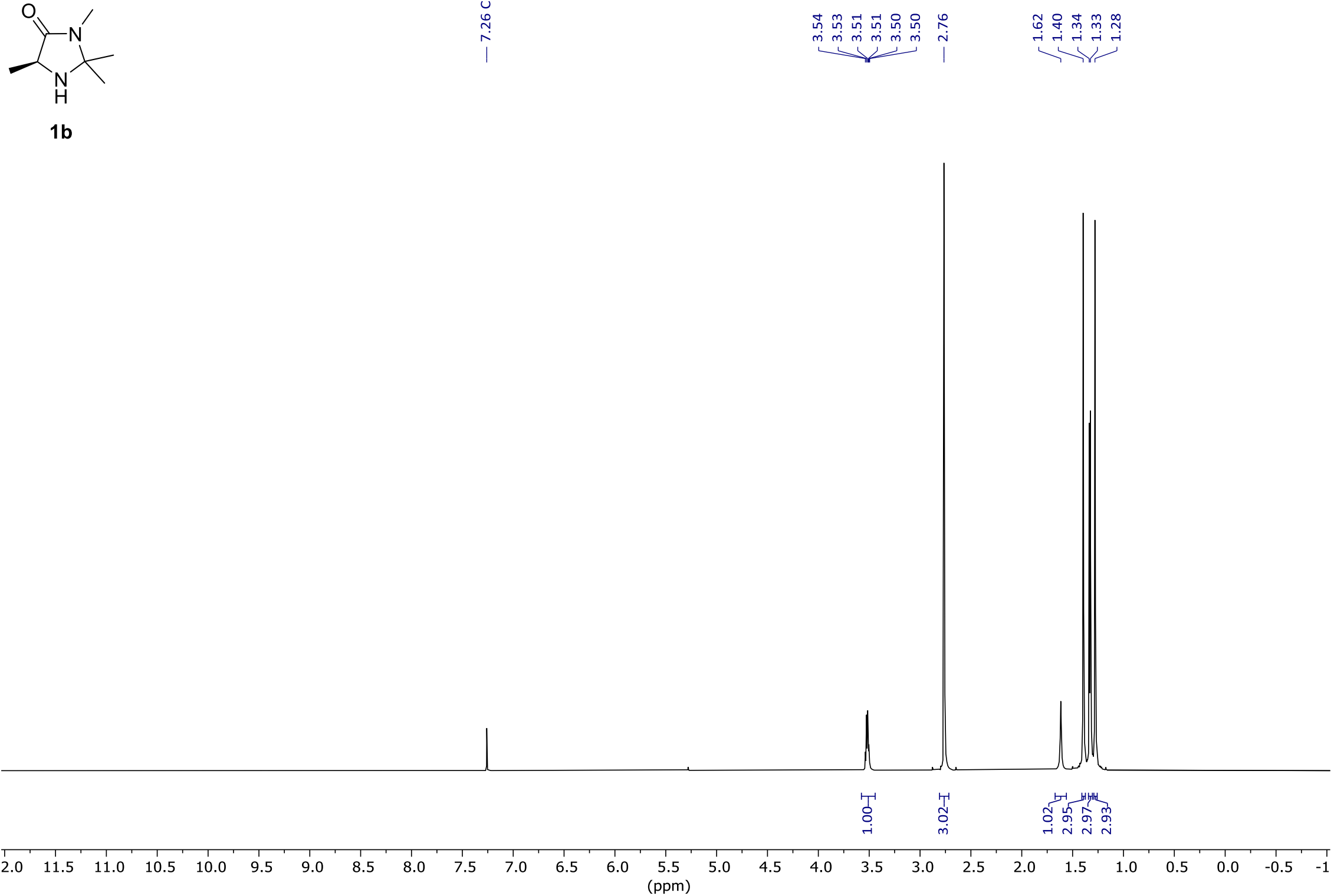
1H NMR, $\mathrm{CDCl} 3,600 \mathrm{MHz}$

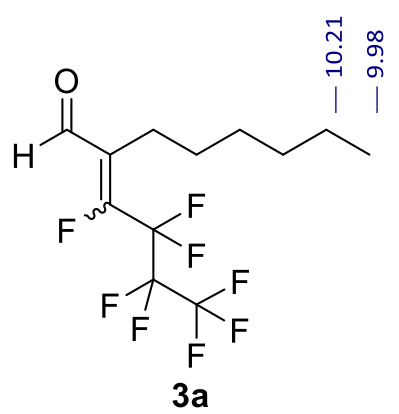

商

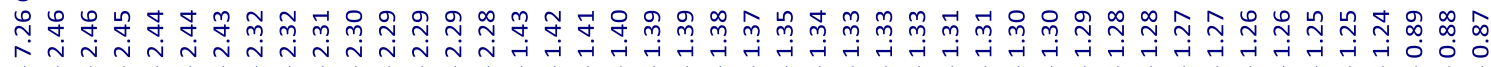

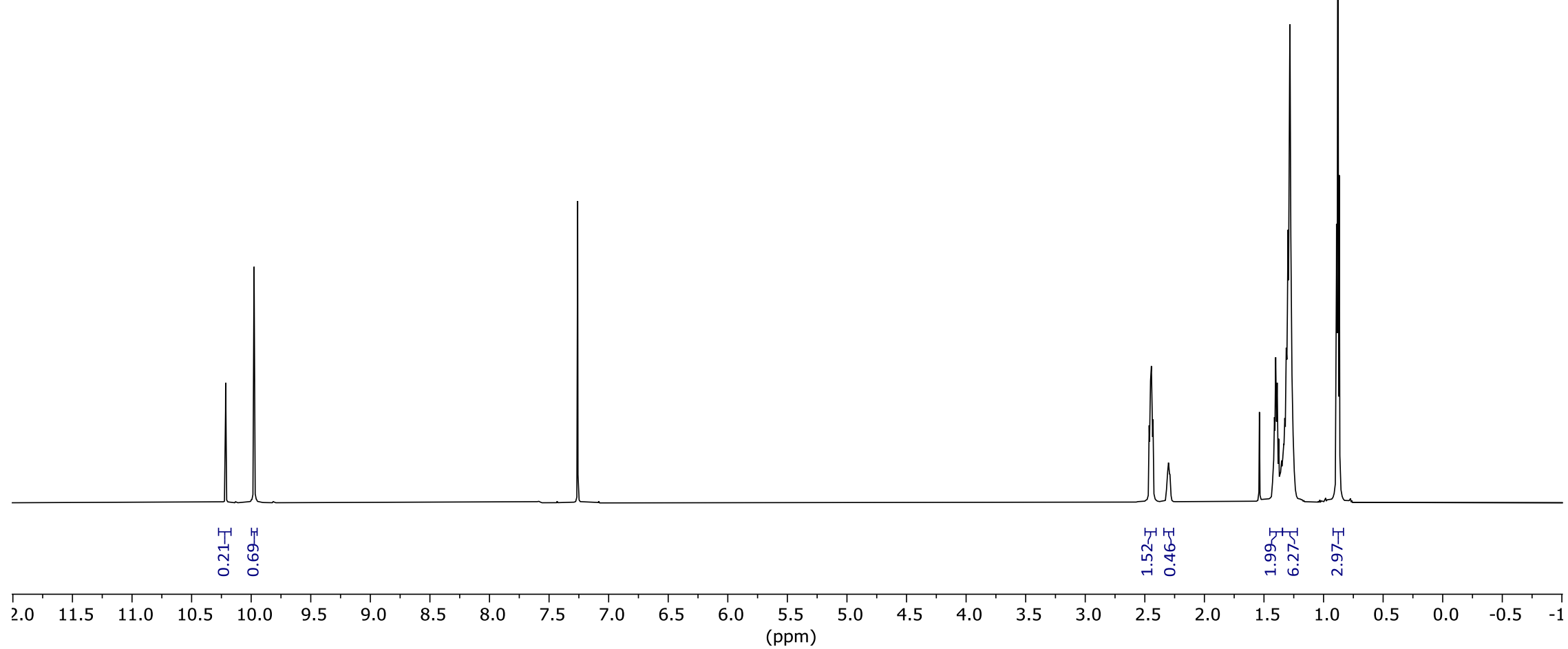




\section{C $\{1 \mathrm{H}\} \mathrm{NMR}, \mathrm{CDCl} 3,75 \mathrm{MHz}$}

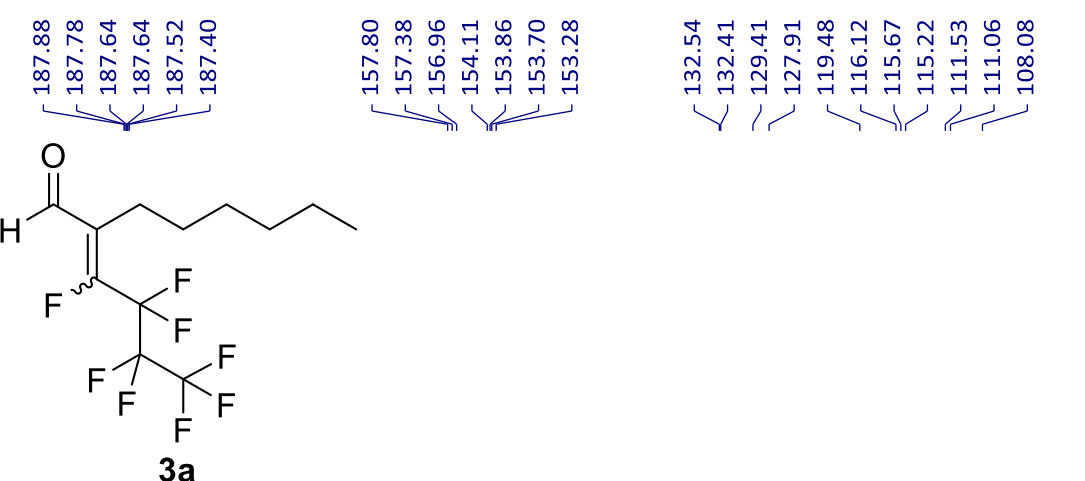

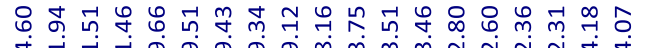

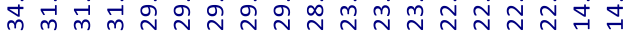

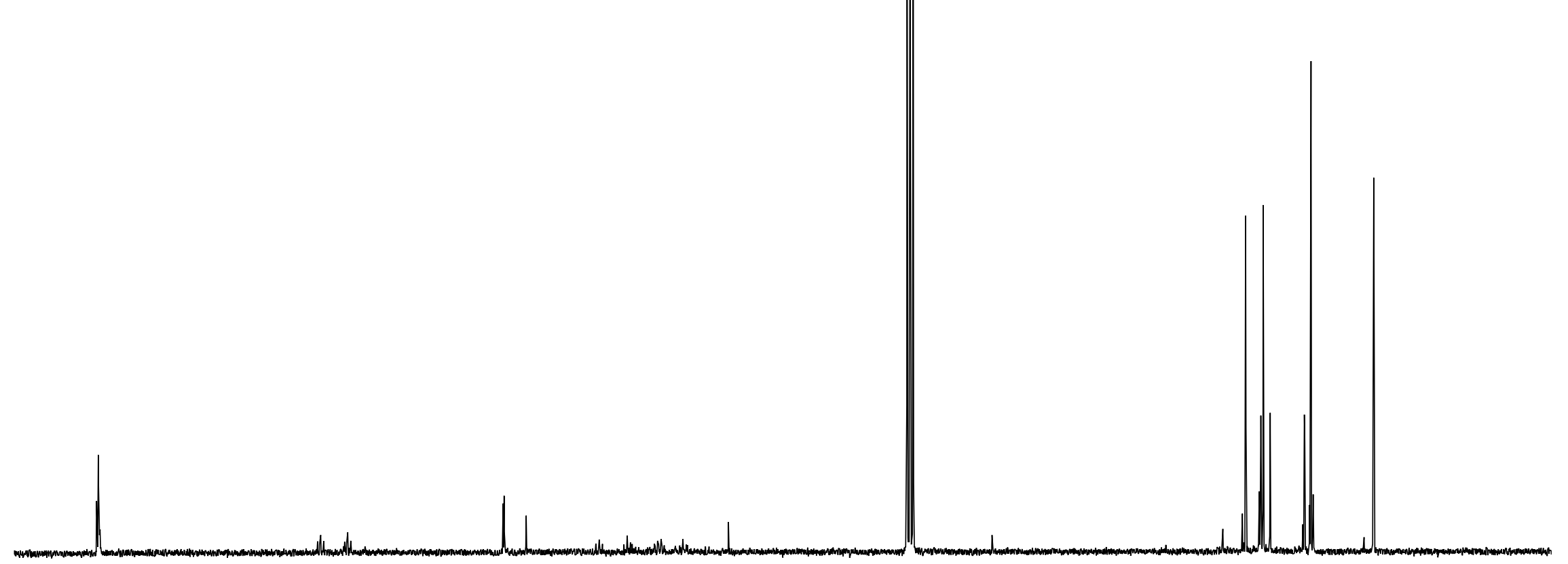

$00 \quad 190+180$ 
19F NMR, $\mathrm{CDCl} 3,282 \mathrm{MHz}$

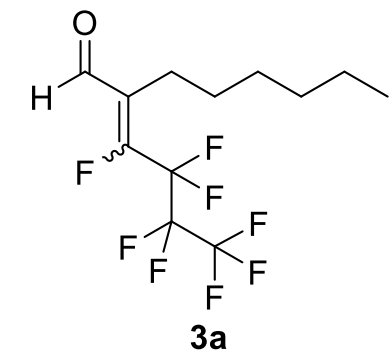

ํํำ

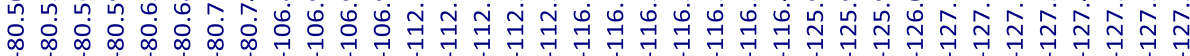

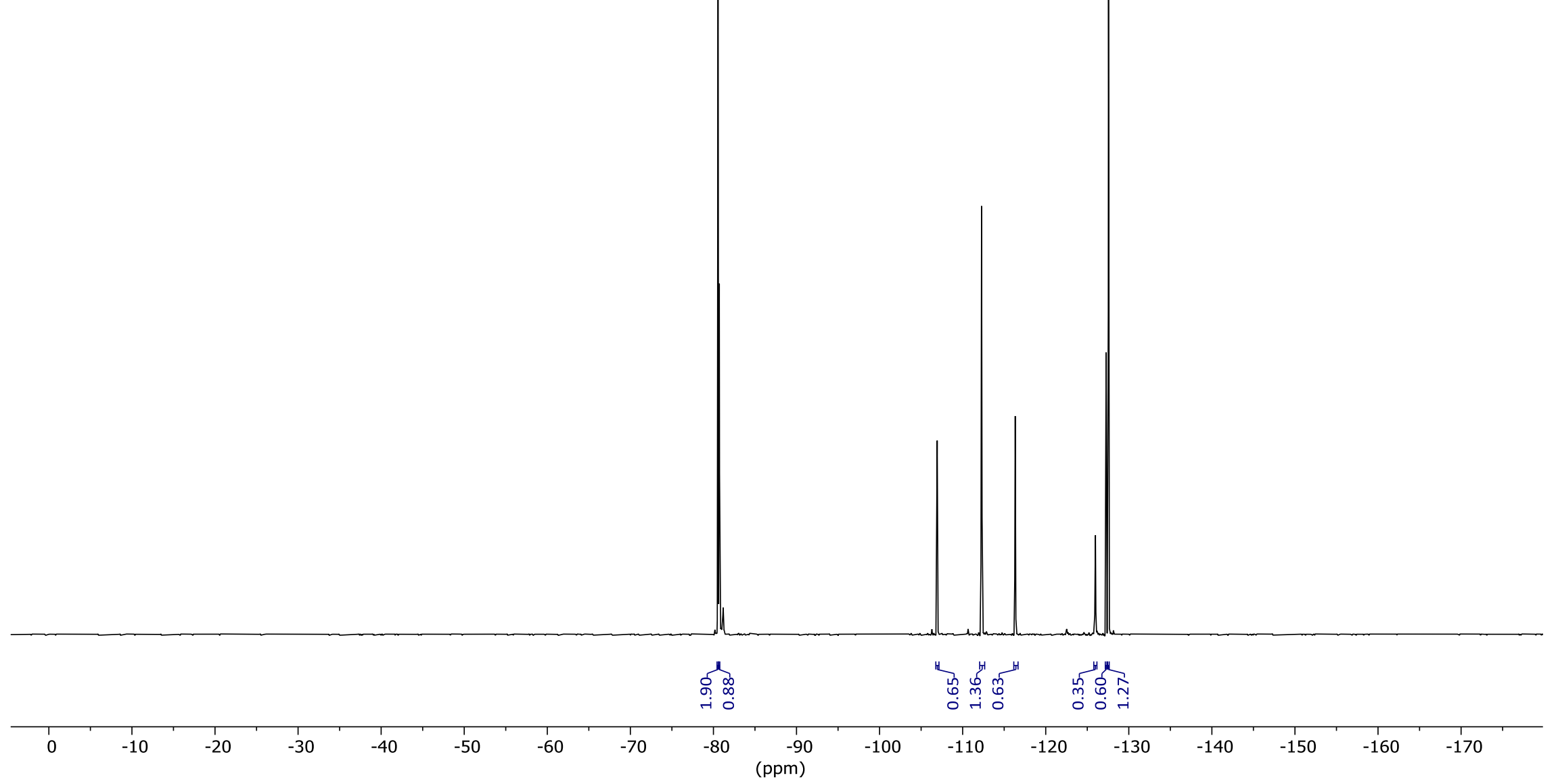




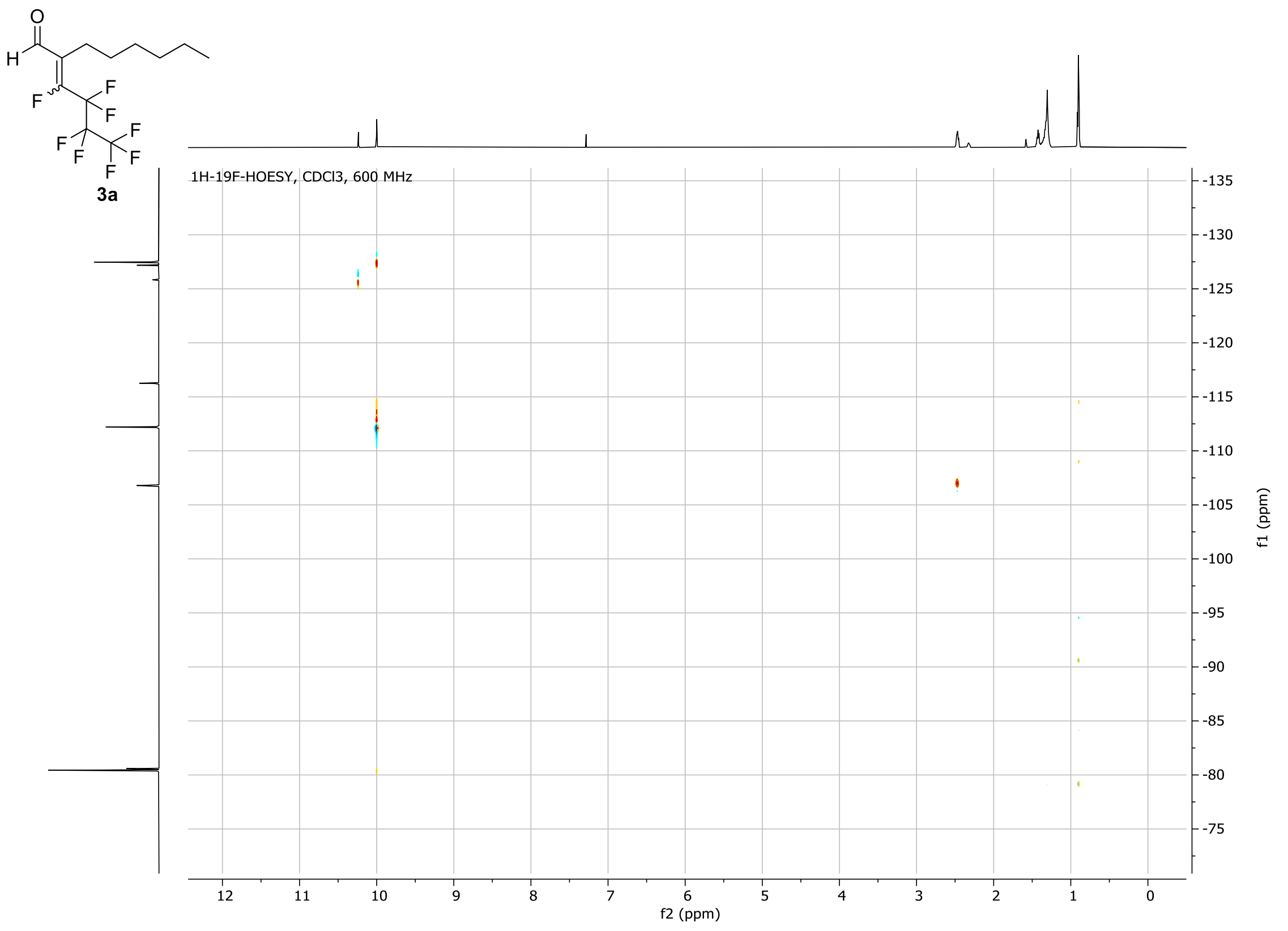


$1 \mathrm{H} \mathrm{NMR}, \mathrm{CDCl} 3,300 \mathrm{MHz}$

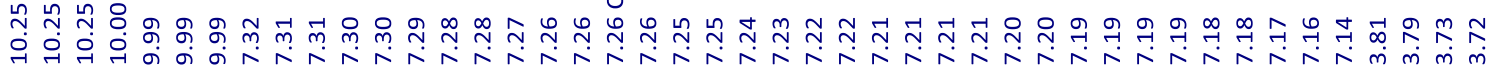

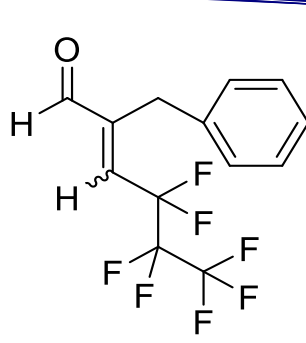

$3 b$

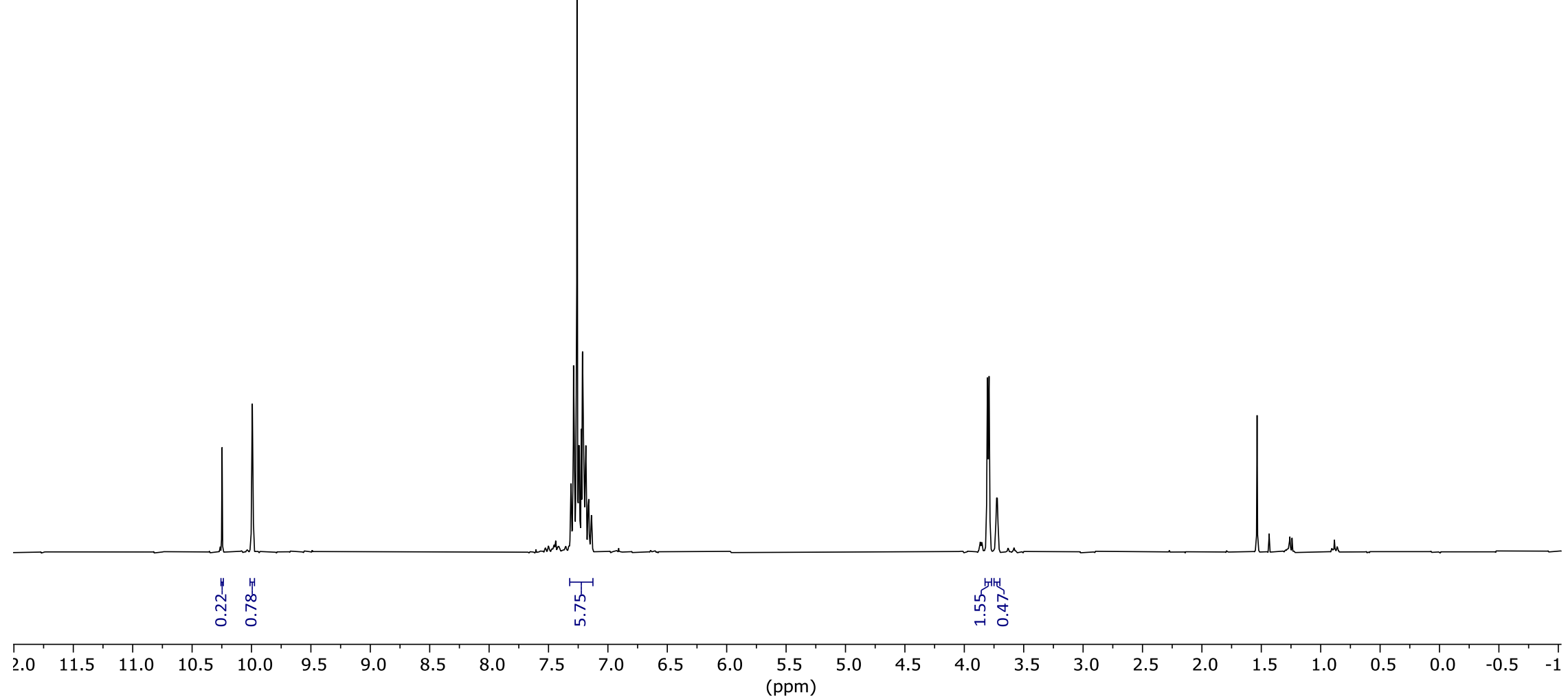


$13 \mathrm{C}\{1 \mathrm{H}\} \mathrm{NMR}, \mathrm{CDCl} 3,151 \mathrm{MHz}$

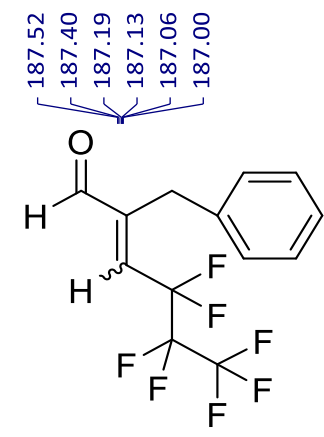

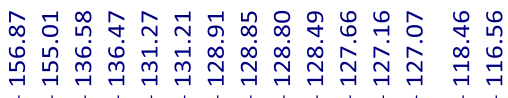

3b

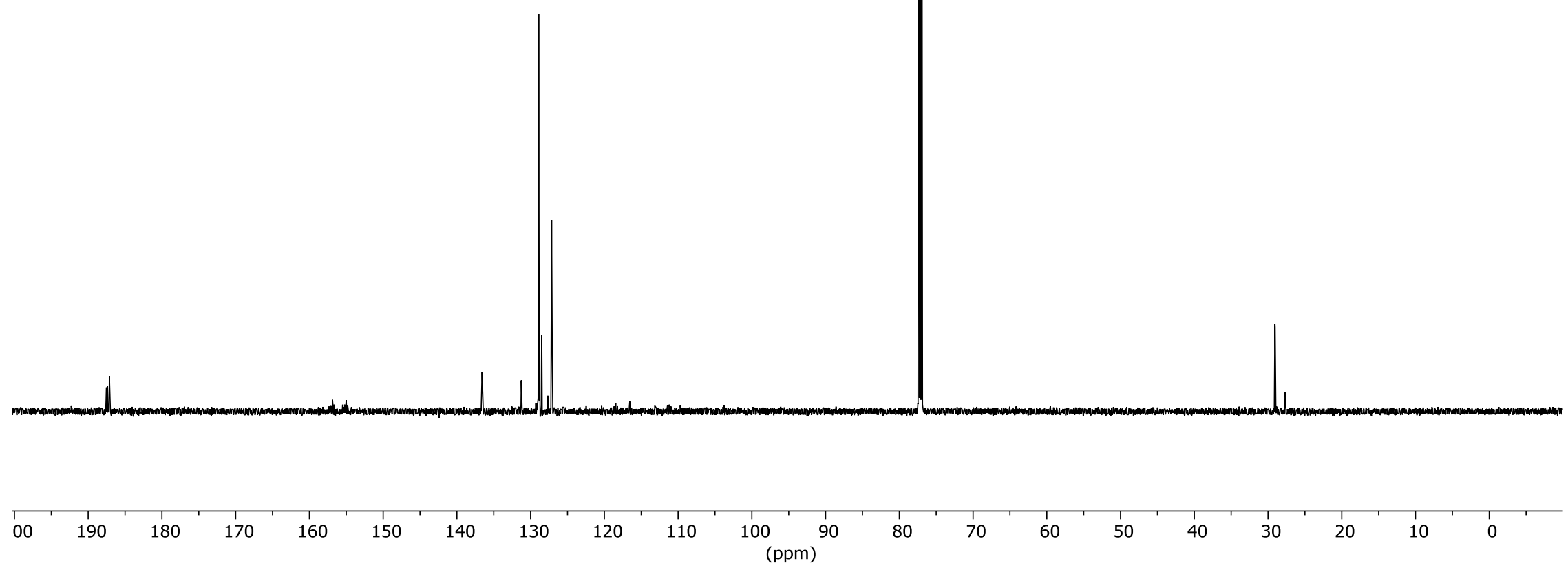


19F NMR, CDCl3, $282 \mathrm{MHz}$

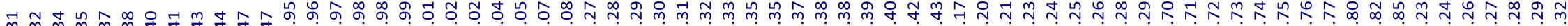

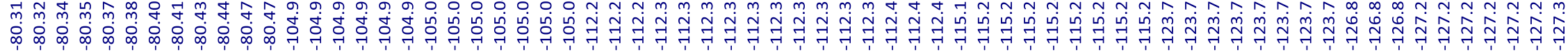

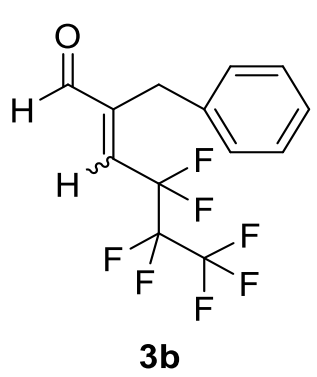

3b

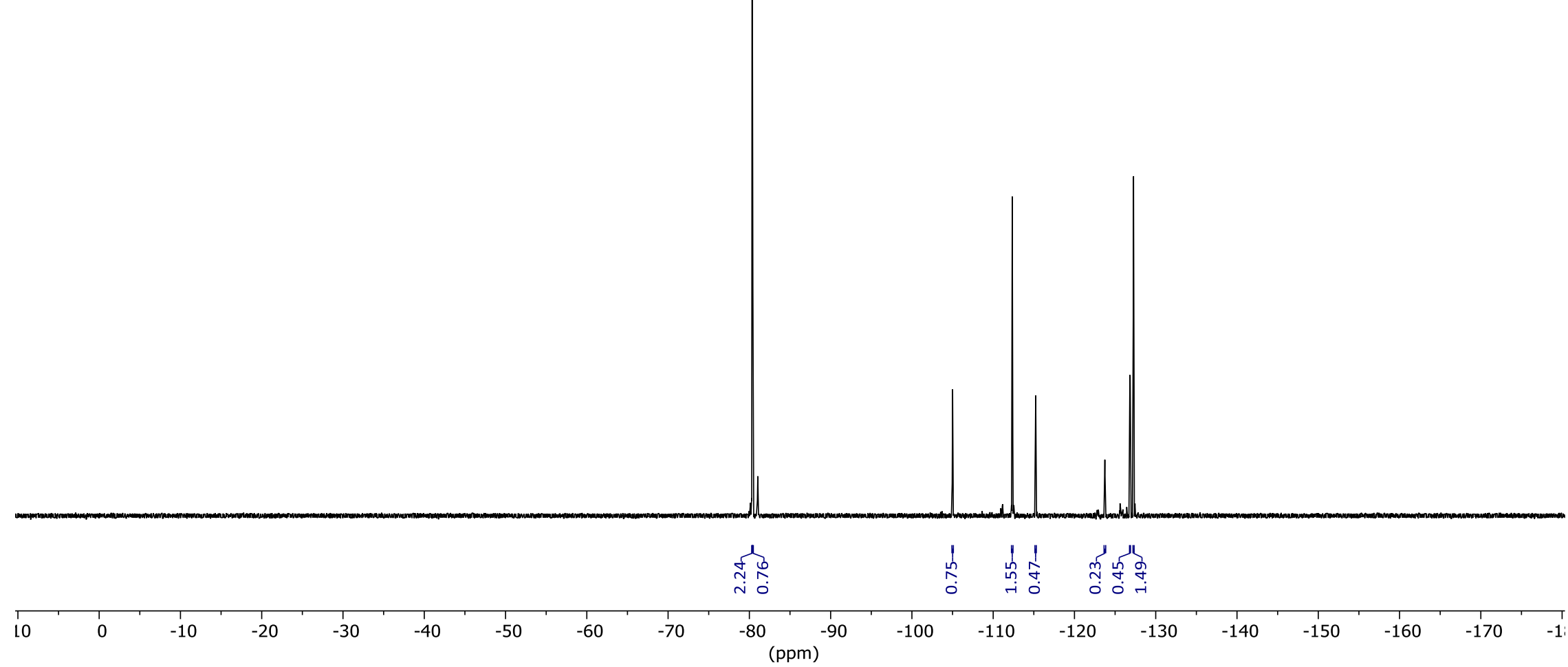




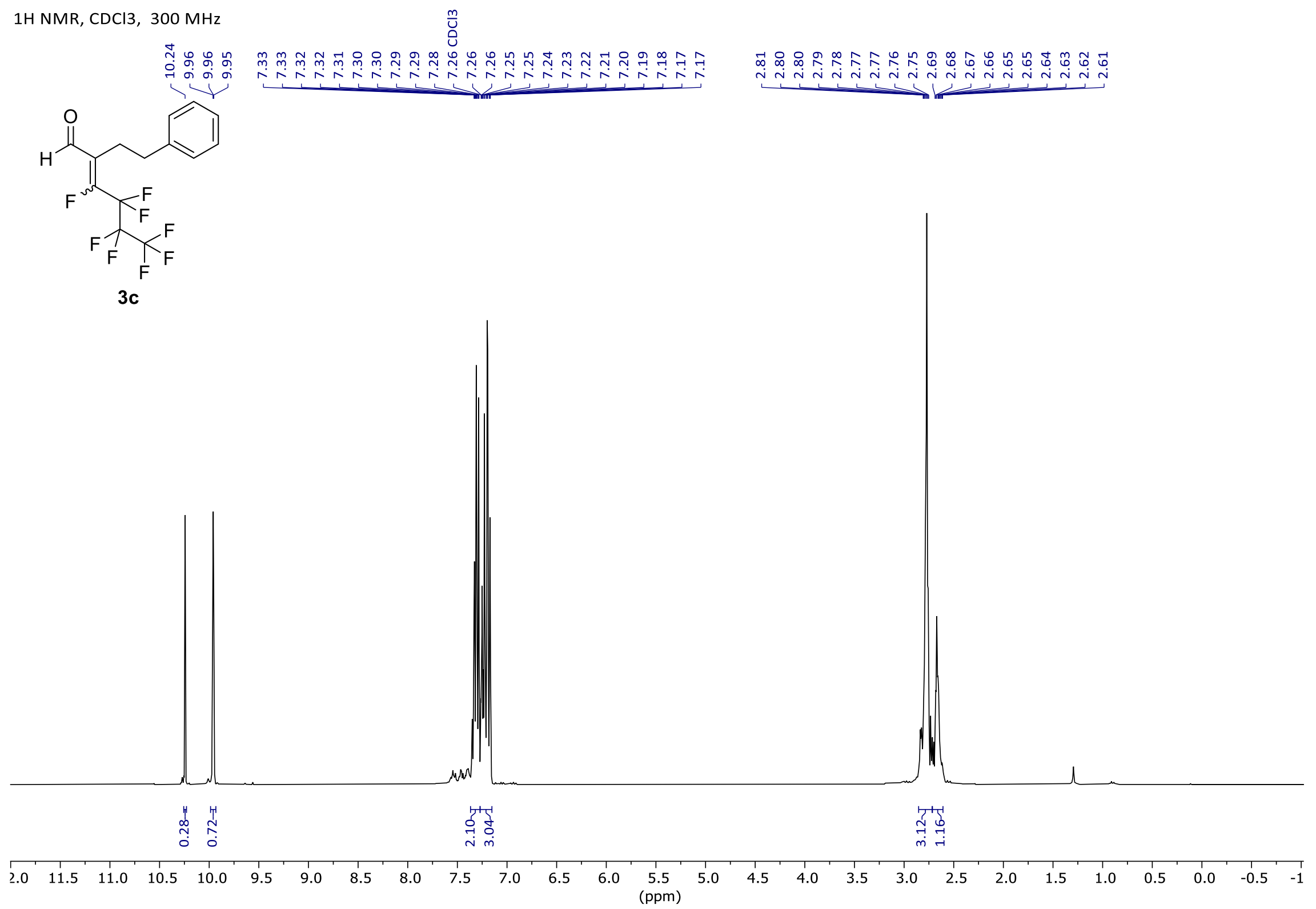


13C $\{1 \mathrm{H}\} \mathrm{NMR}, \mathrm{CDCl} 3,75 \mathrm{MHz}$

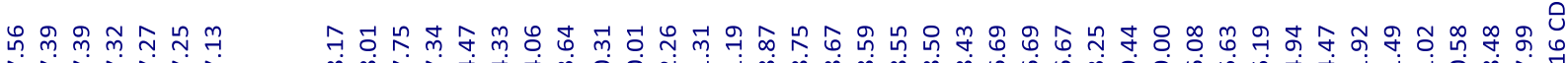

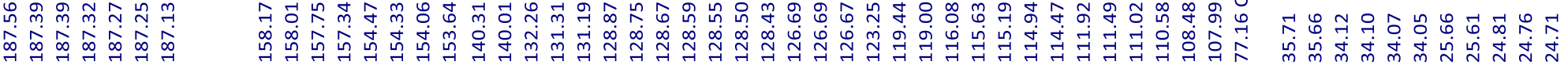

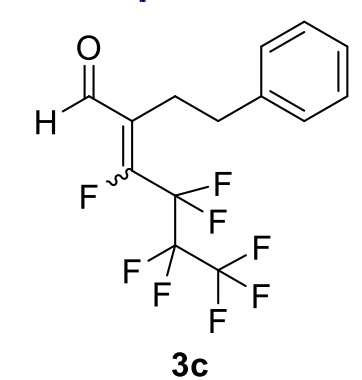

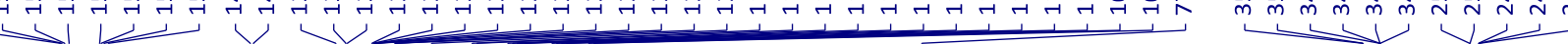

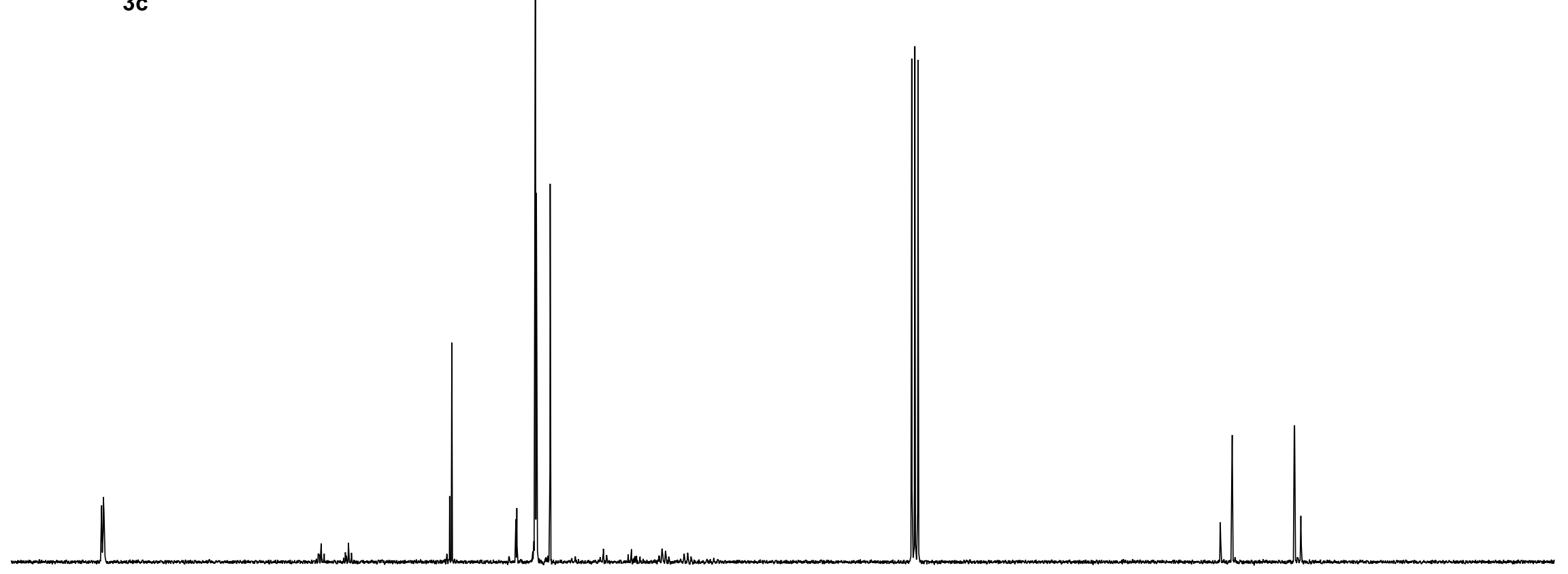

$190 \quad 180 \quad 170 \quad 160 \quad 150 \quad 140$

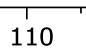


19F NMR, CDCl3, $282 \mathrm{MHz}$

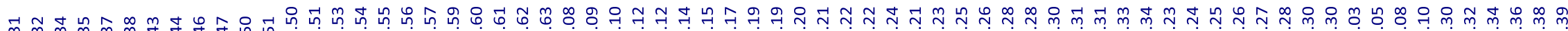

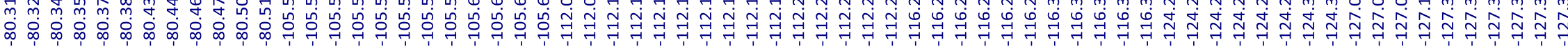
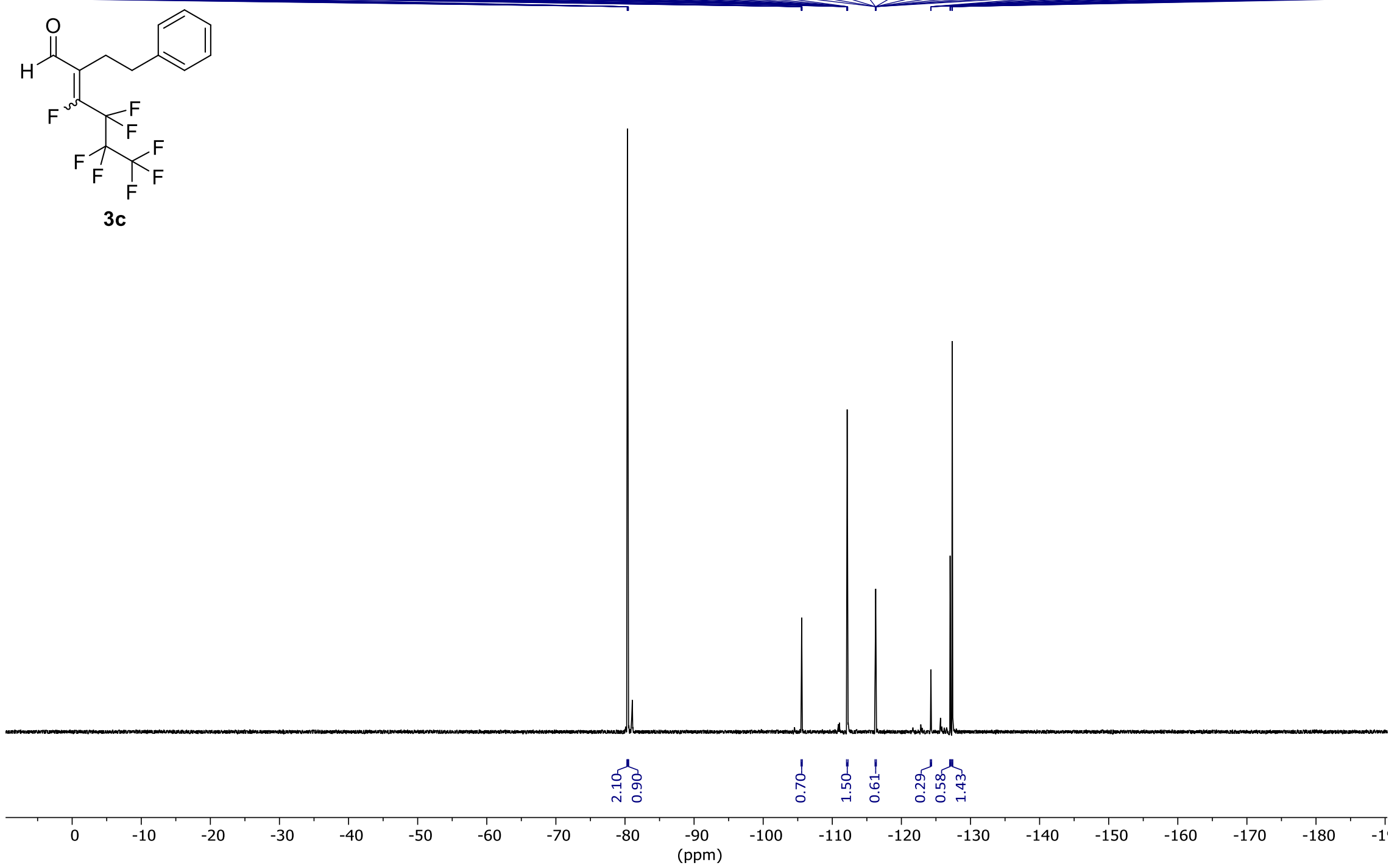
$1 \mathrm{H} \mathrm{NMR,} \mathrm{CDCl} 3,300 \mathrm{MHz}$

每

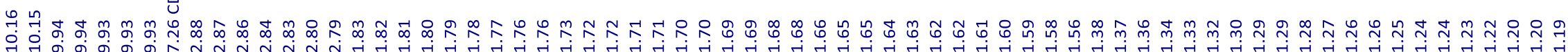

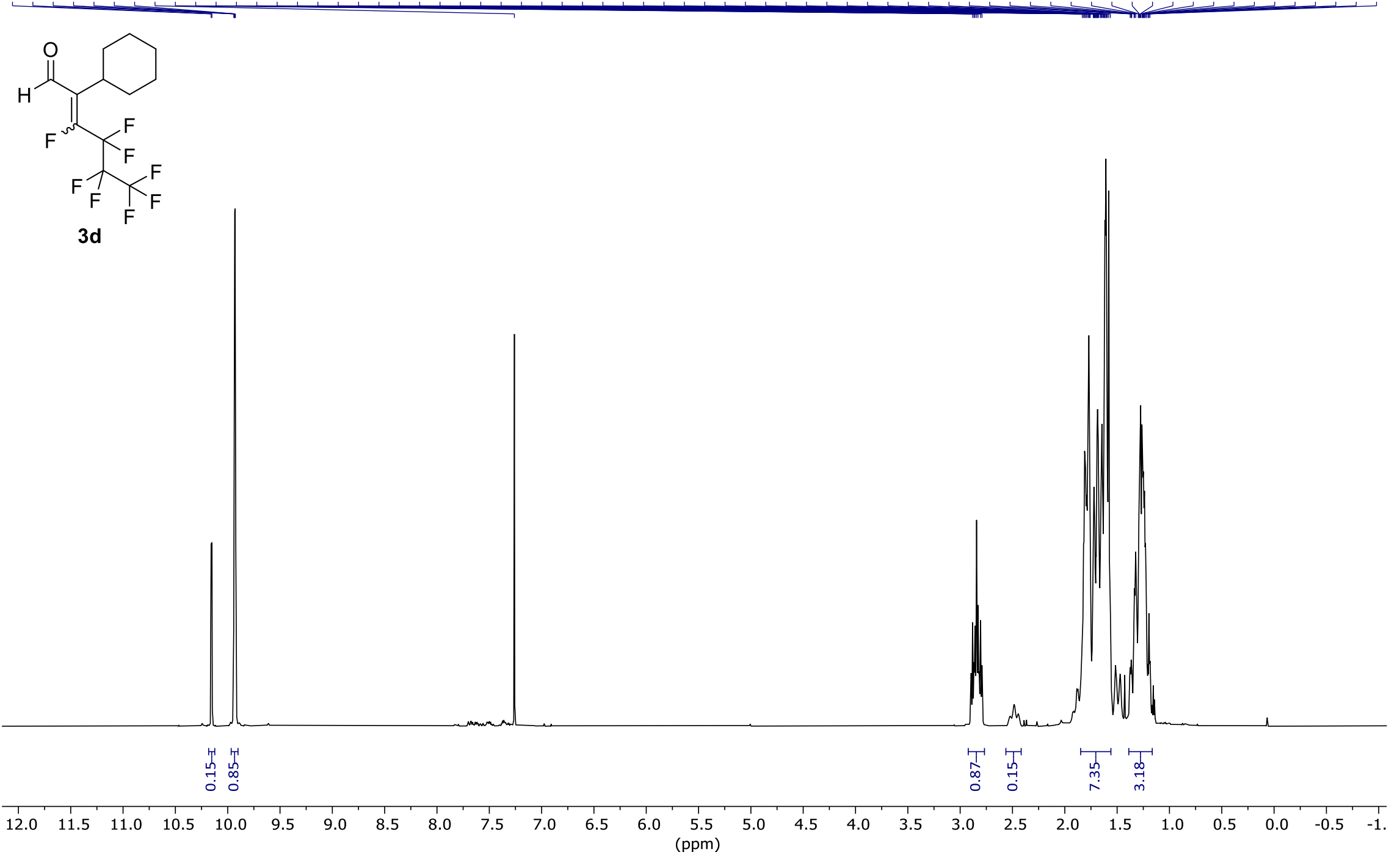


$13 \mathrm{C}\{1 \mathrm{H}\} \mathrm{NMR}, \mathrm{CDCl} 3,75 \mathrm{MHz}$

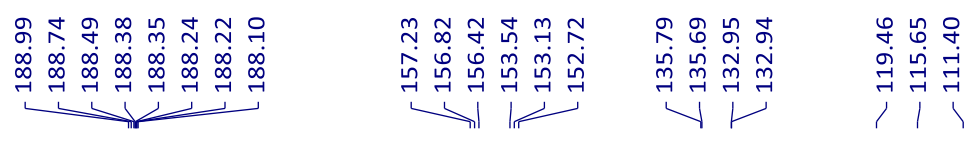

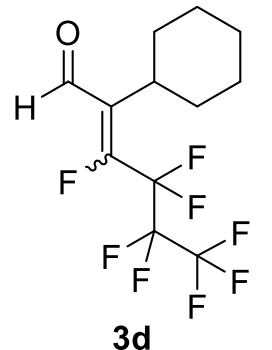

m

ம்

1
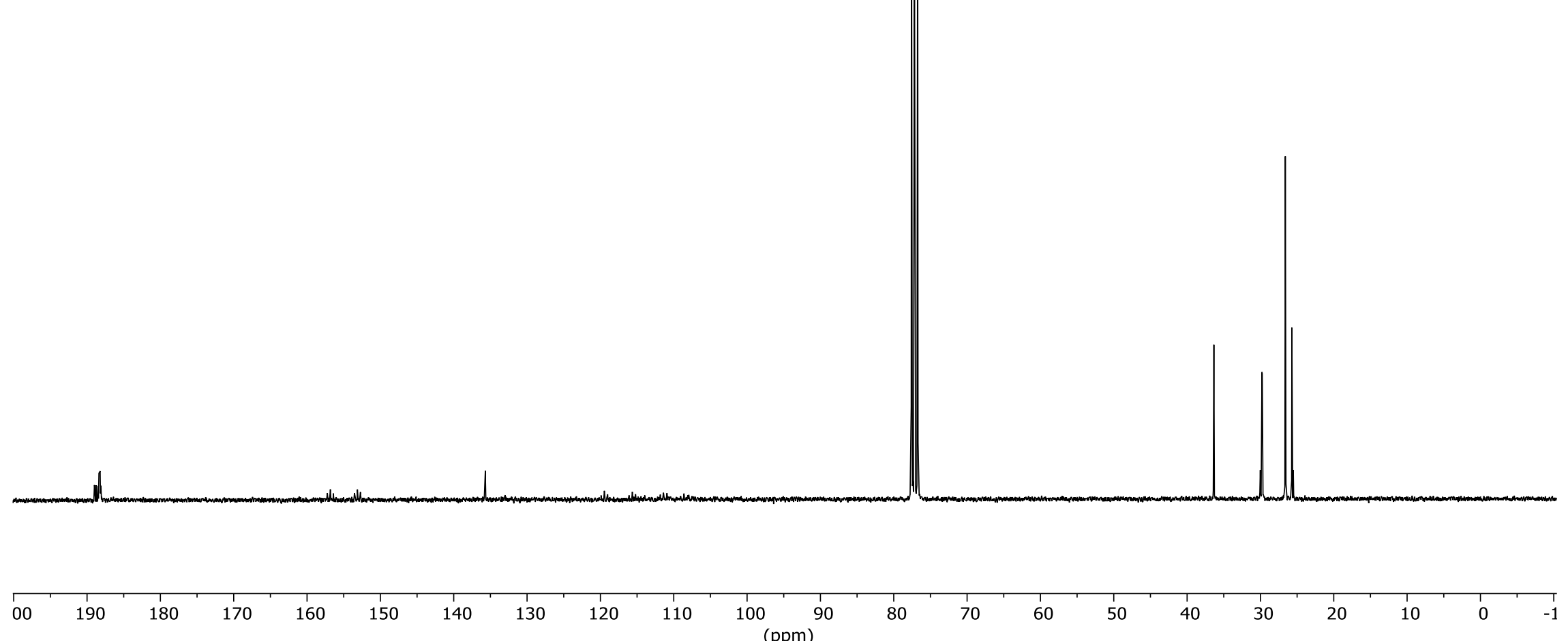
19F NMR, CDCl3, $282 \mathrm{MHz}$

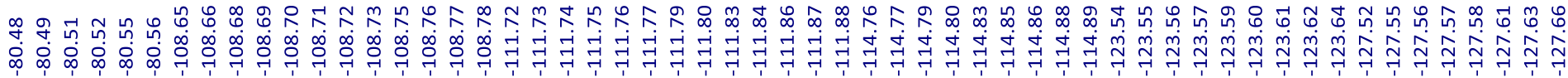

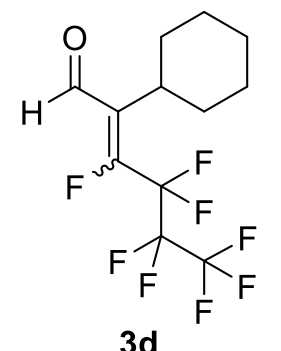

$3 d$

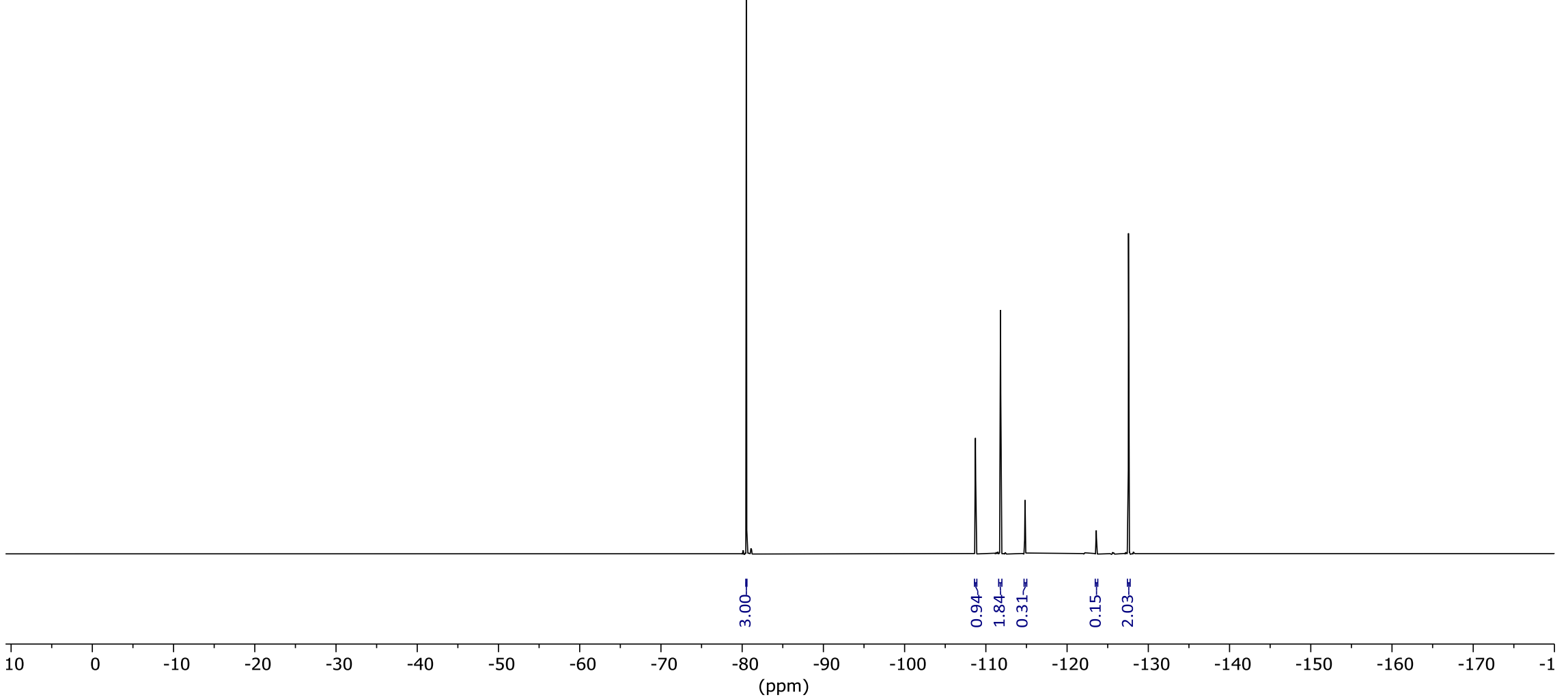



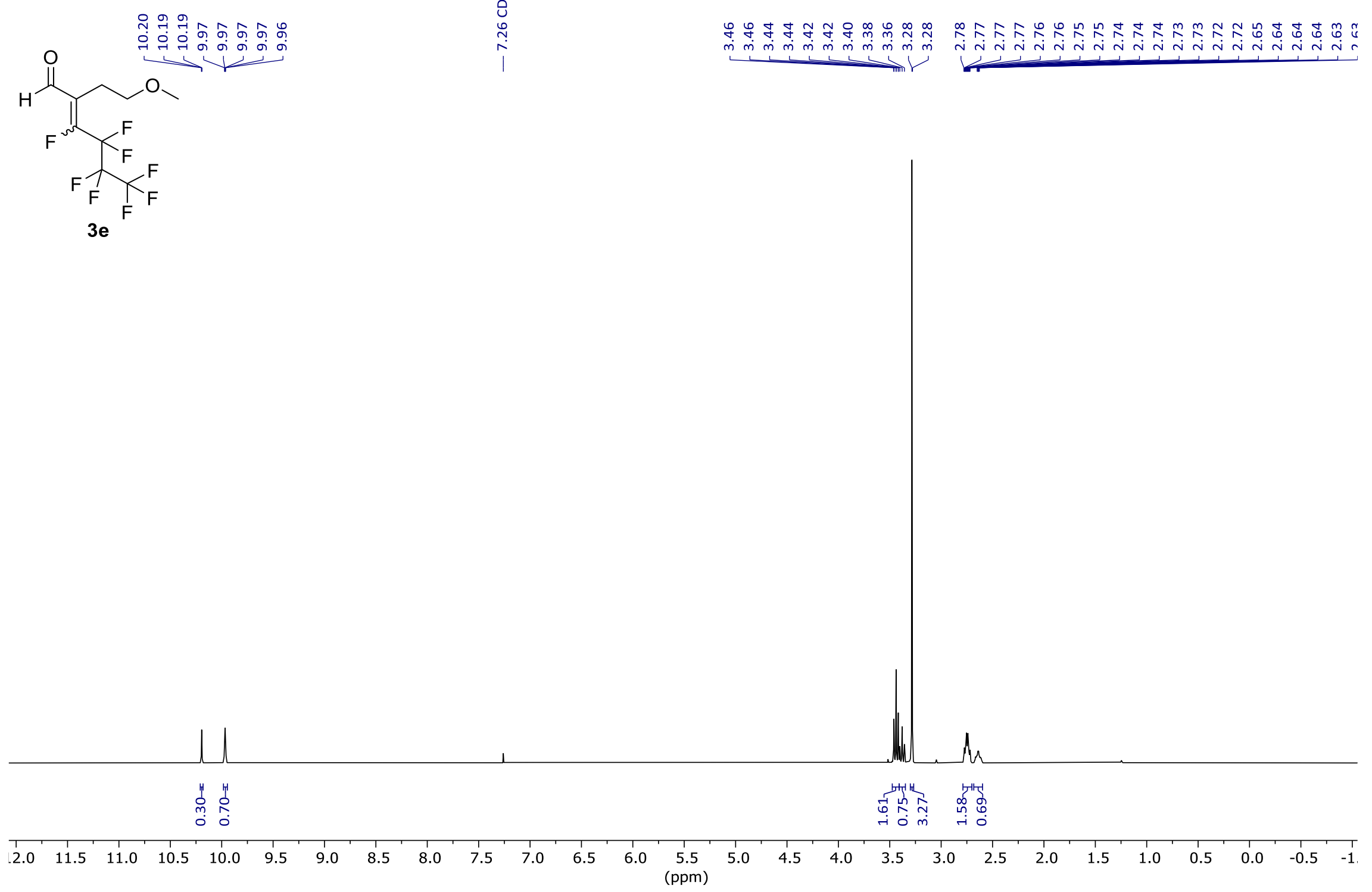


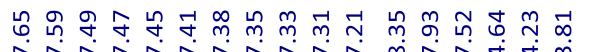

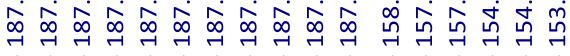

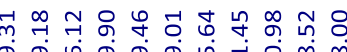

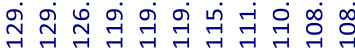

品

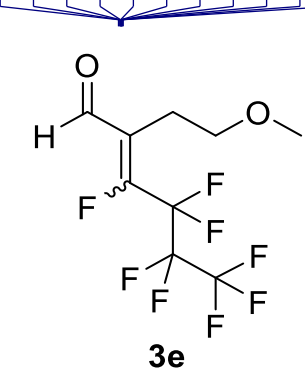

$3 e$

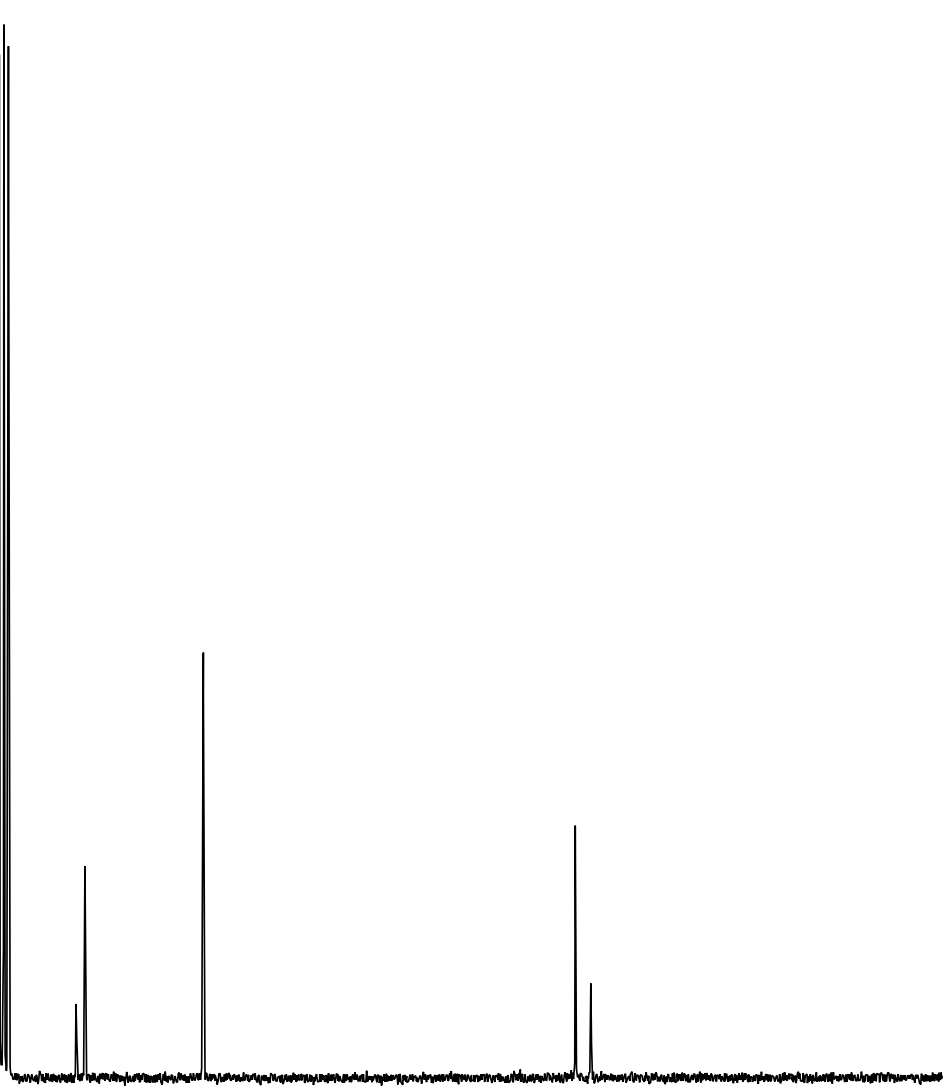

$190 \quad 180$

160

140

130

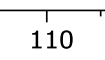


19F NMR, $\mathrm{CDCl} 3,282 \mathrm{MHz}$

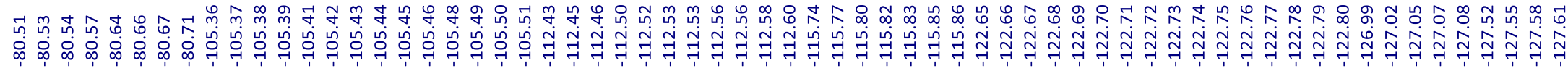

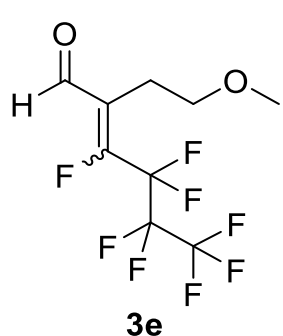

$3 e$

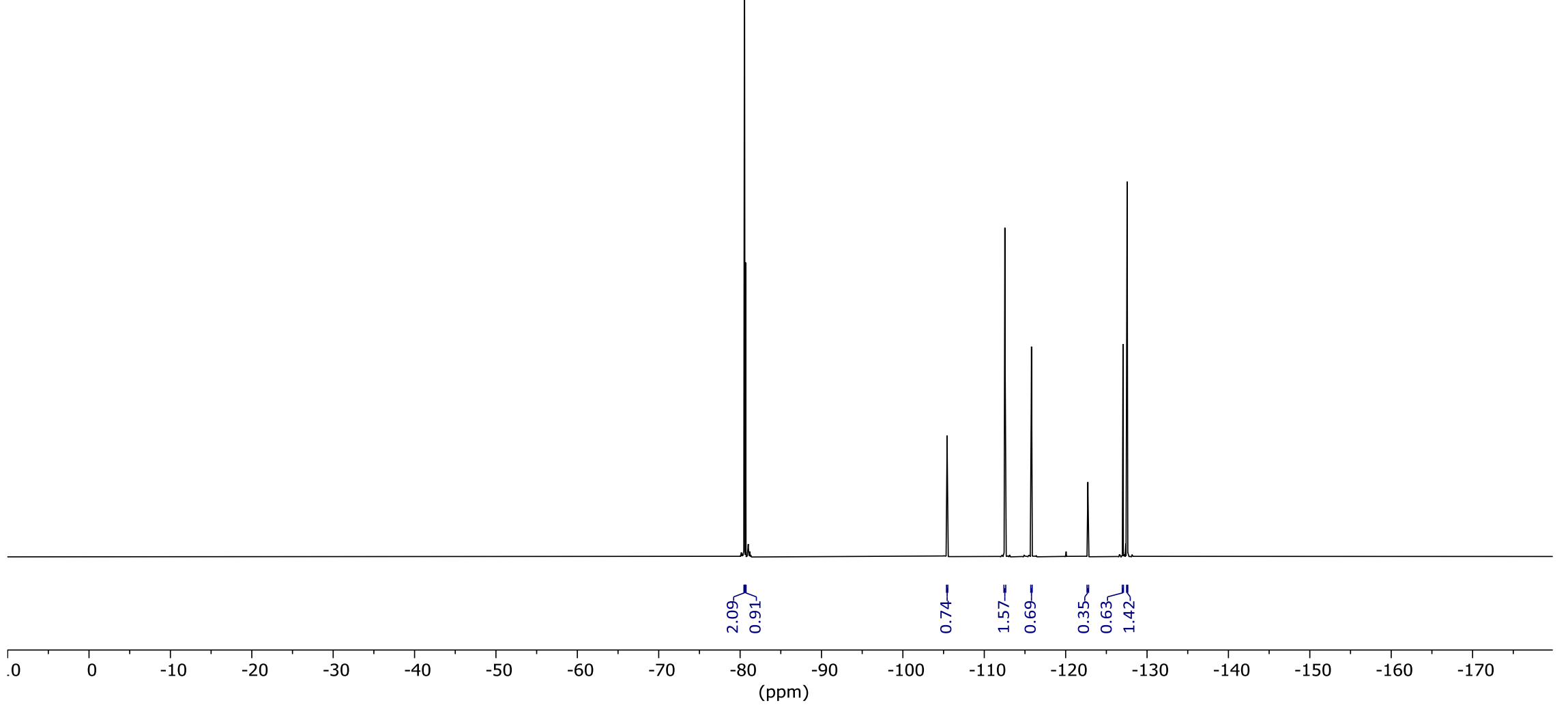


1H NMR, CDCl3, $300 \mathrm{MHz}$

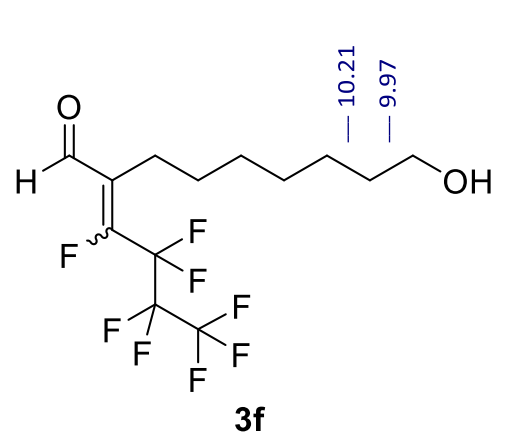

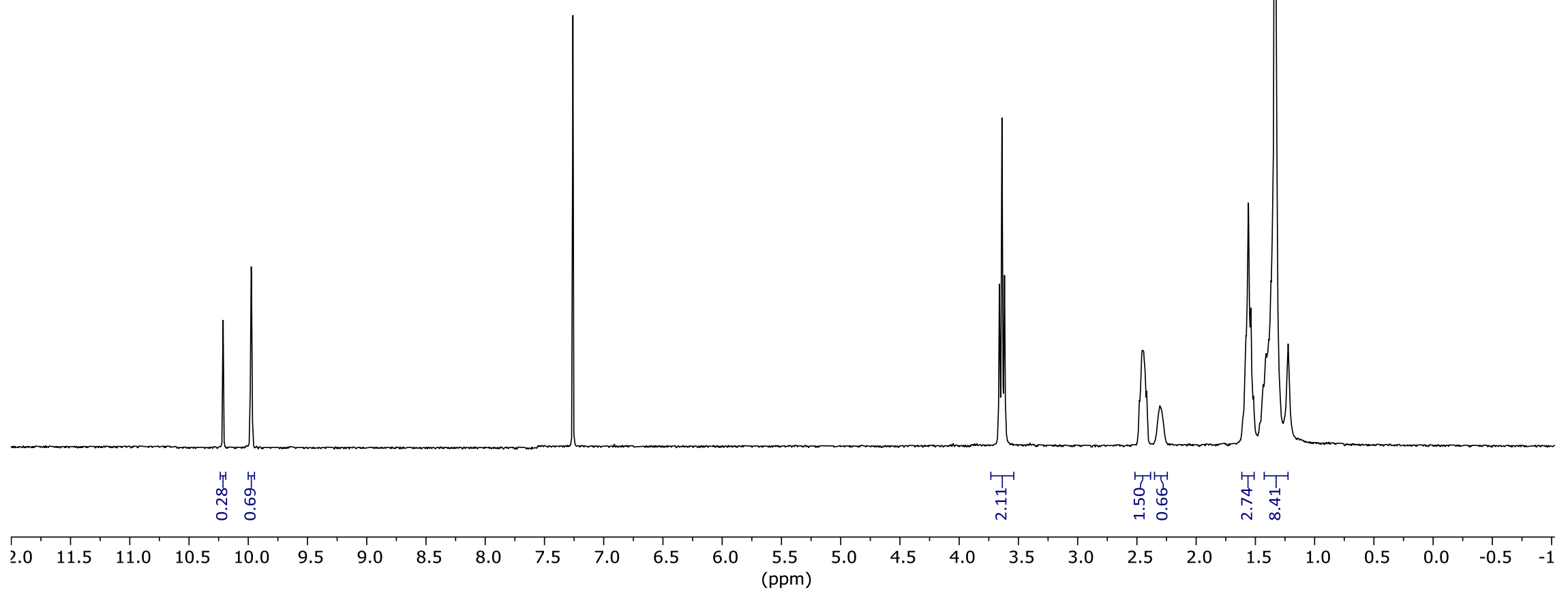


$13 \mathrm{C}\{1 \mathrm{H}\} \mathrm{NMR}, \mathrm{CDCl} 3,75 \mathrm{MHz}$

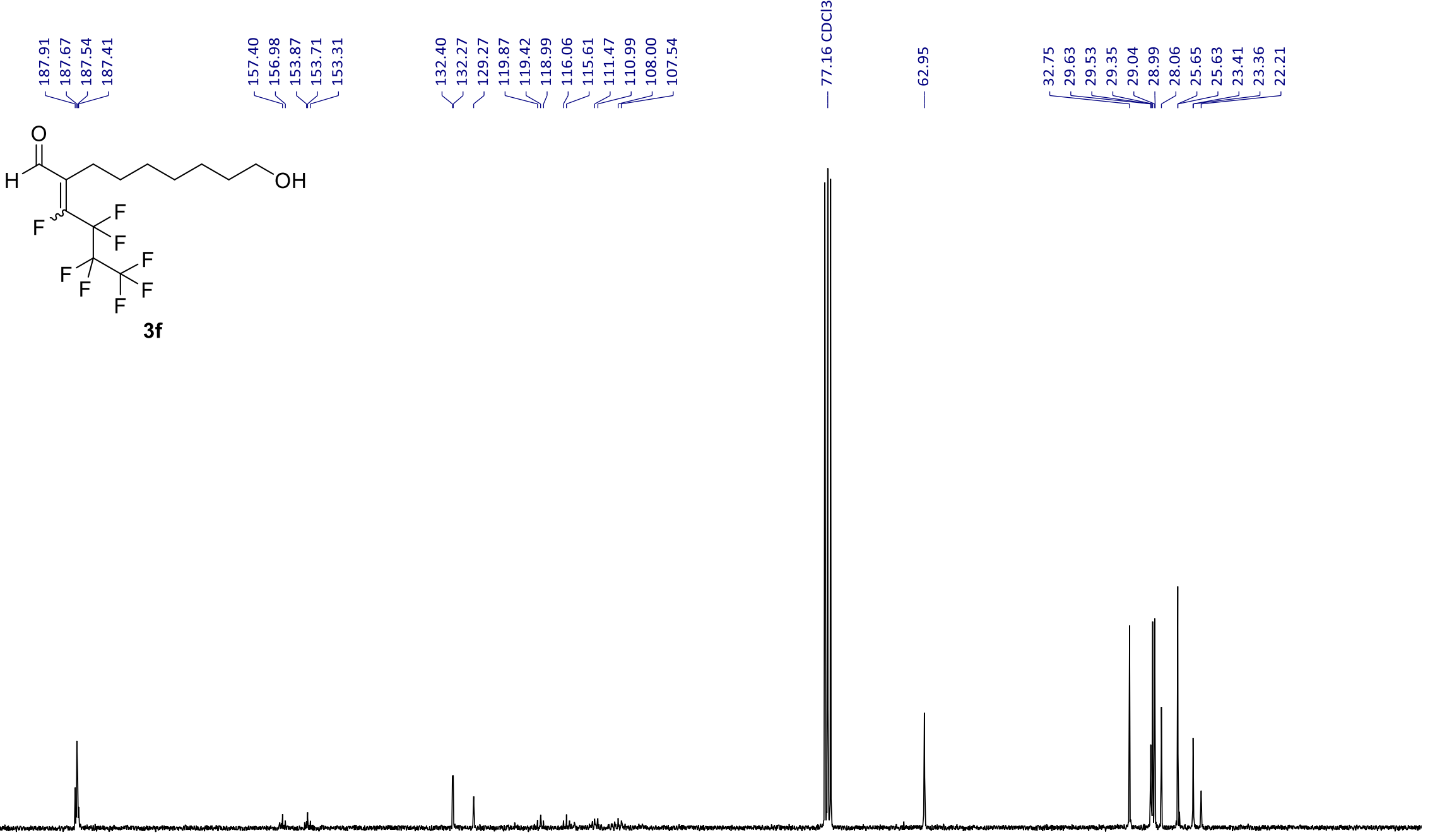

\begin{tabular}{lllllllllllllllllllllll}
\hline & 00 & 190 & 180 & 170 & 160 & 150 & 140 & 130 & 120 & 110 & 100 & 90 & 80 & 70 & 60 & 50 & 40 & 30 & 20 & 10 & 0 & -1
\end{tabular} 
19F NMR, $\mathrm{CDCl} 3,282 \mathrm{MHz}$

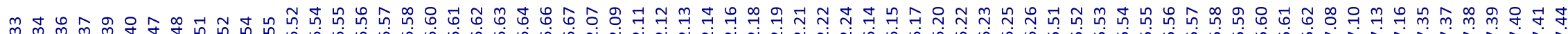

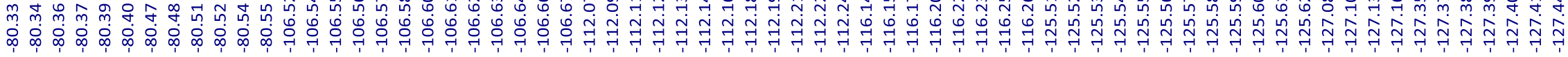
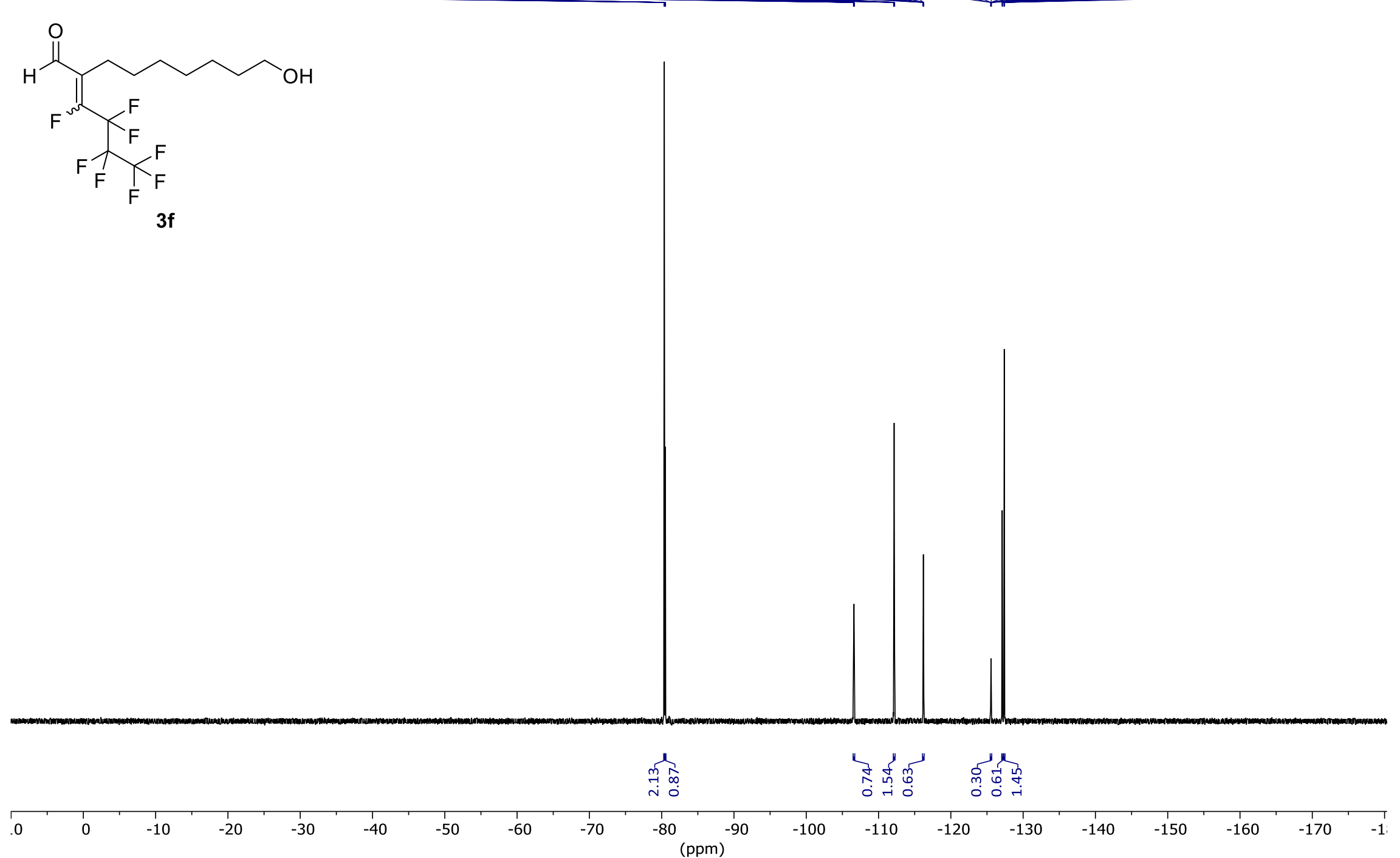
1H NMR, $\mathrm{CDCl} 3,600 \mathrm{MHz}$

范

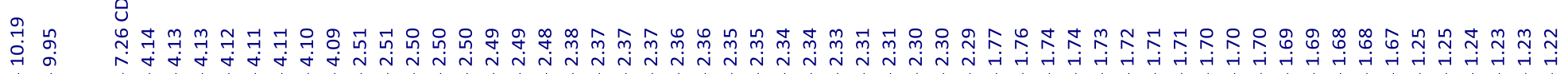<smiles>CCOC(=O)CCCC(=CC=O)C(F)(F)C(F)(F)C(F)(F)F</smiles>

$3 \mathrm{~g}$

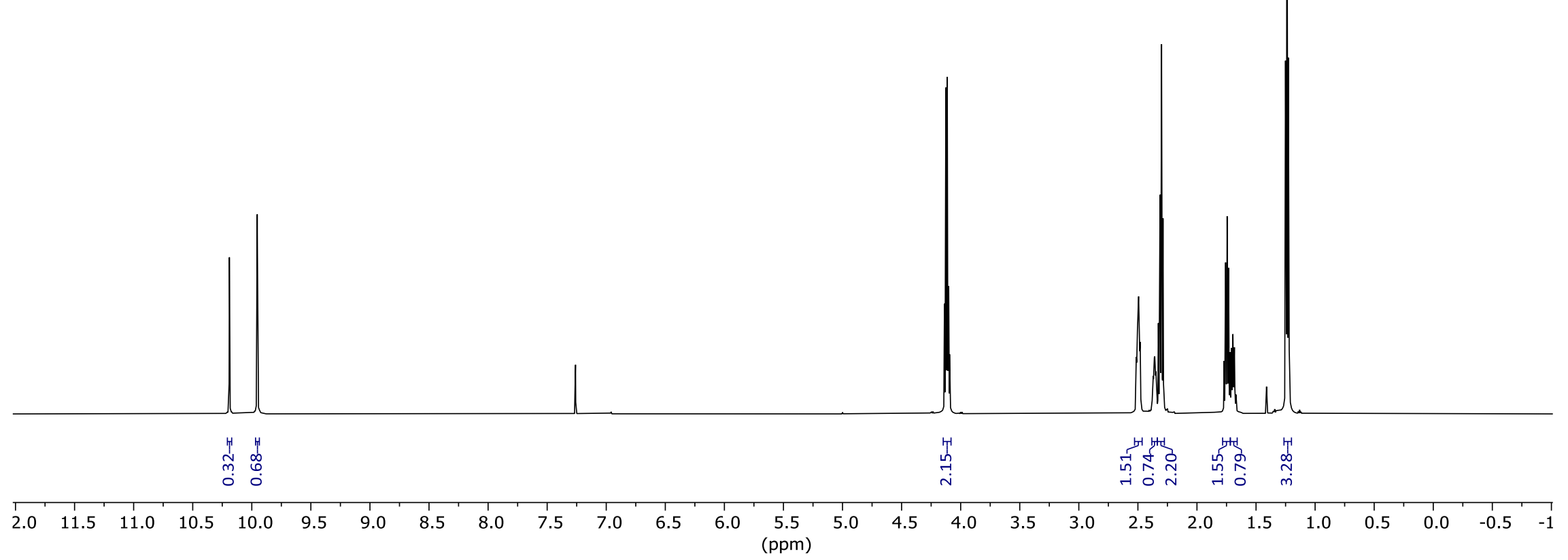


13C $\{1 \mathrm{H}\} \mathrm{NMR}, \mathrm{CDCl} 3,151 \mathrm{MHz}$

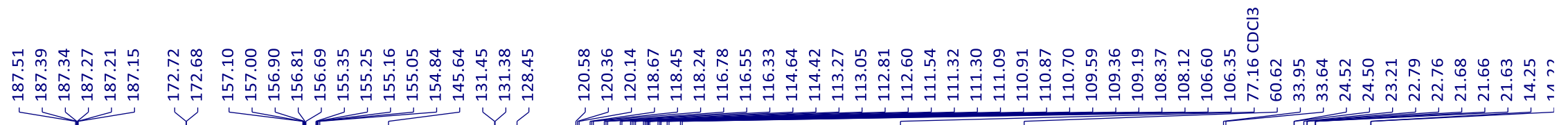
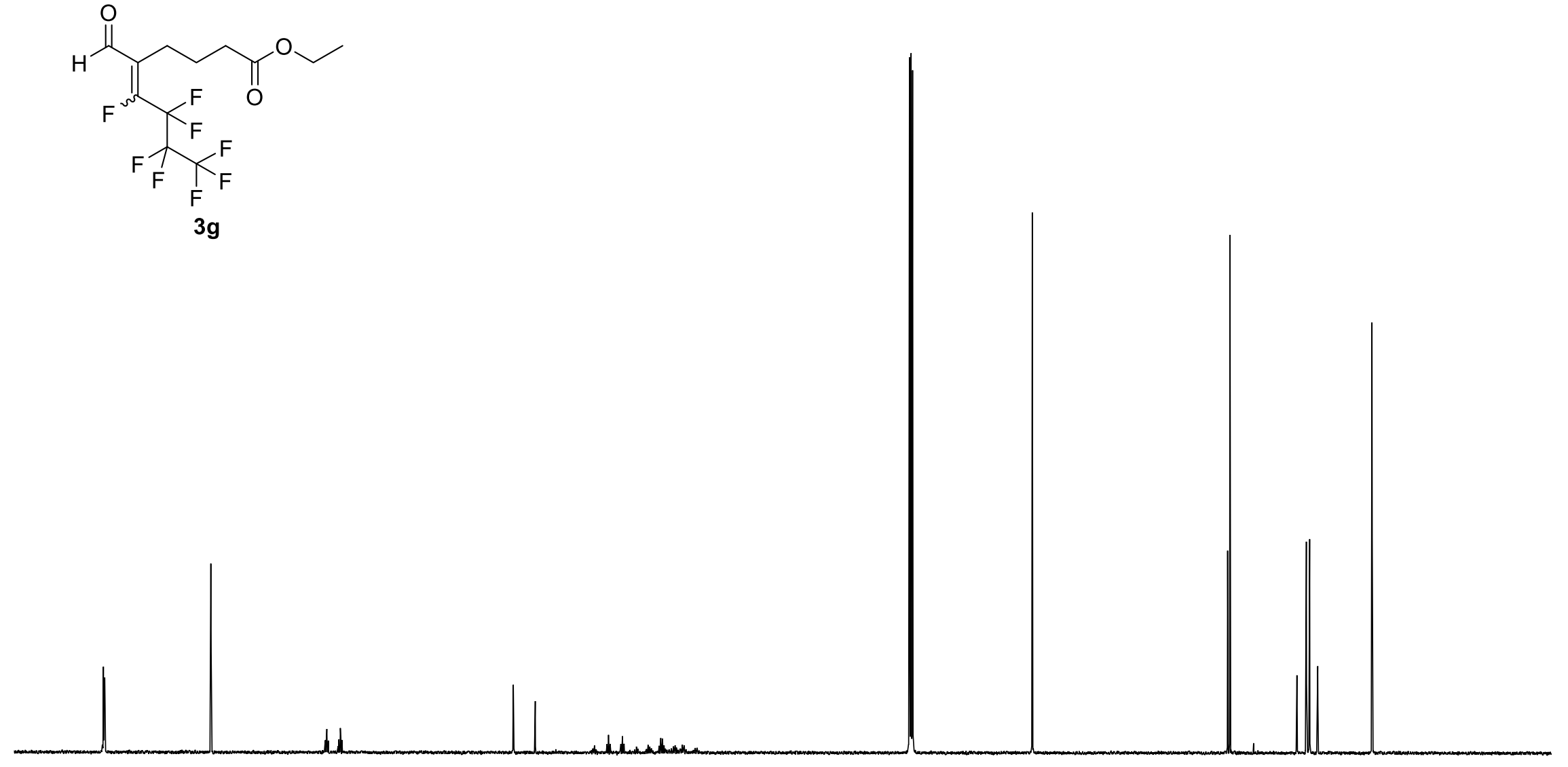

190

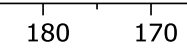

160

140

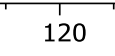

110

(ppm) 
19F NMR, CDCl3, $282 \mathrm{MHz}$

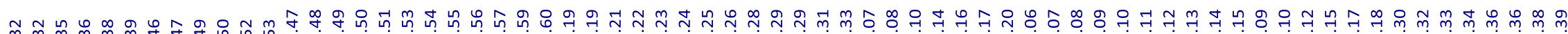

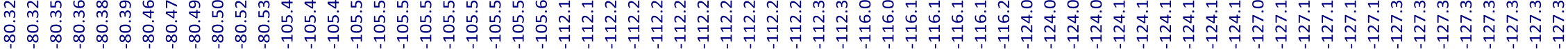<smiles>CCOC(=O)CCC/C=C(/F)C(F)(C(F)(F)F)C(F)(F)F</smiles>

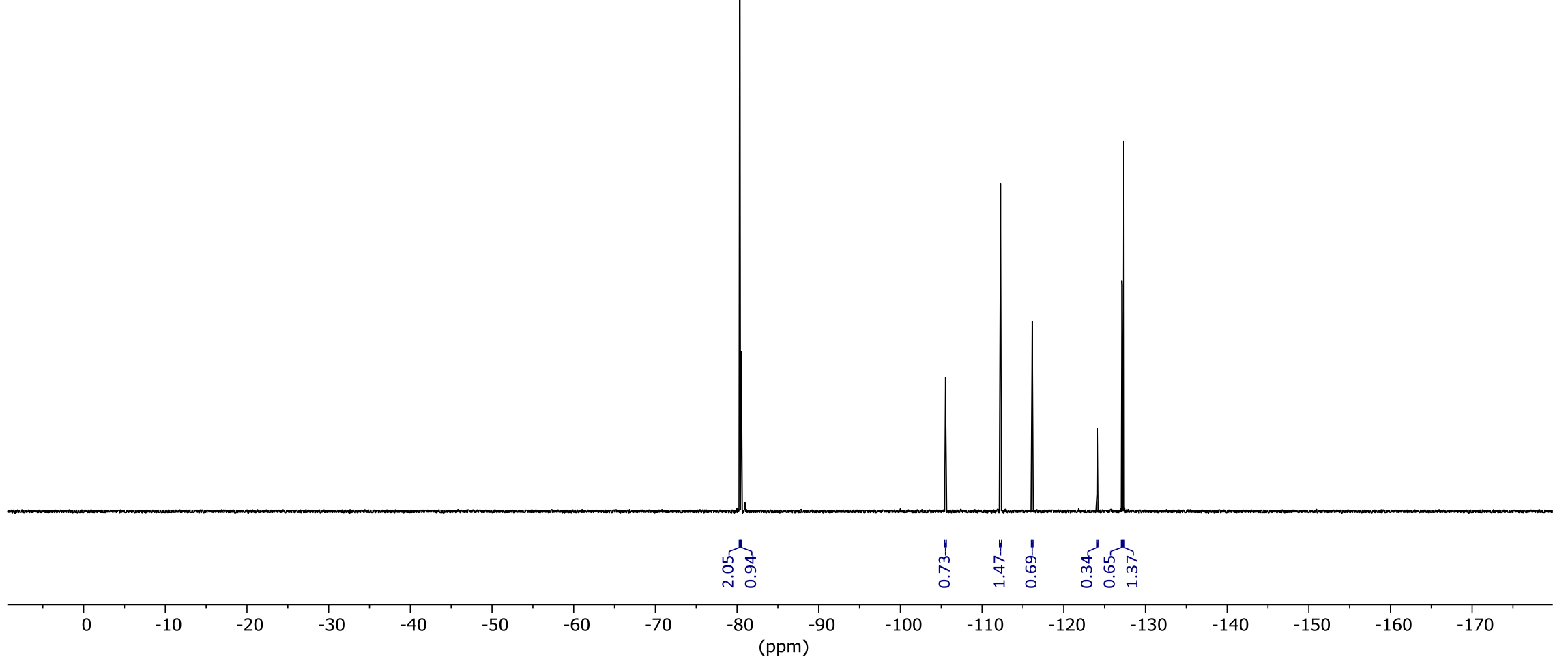


1H NMR, CDCl3, $300 \mathrm{MHz}$

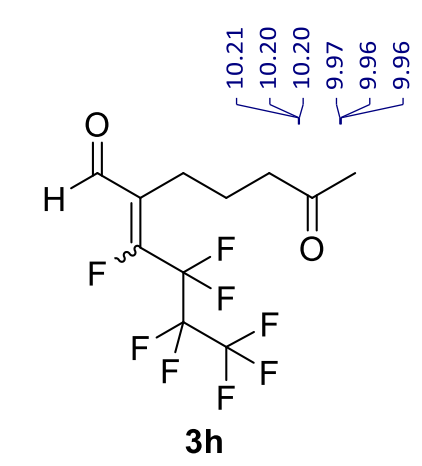

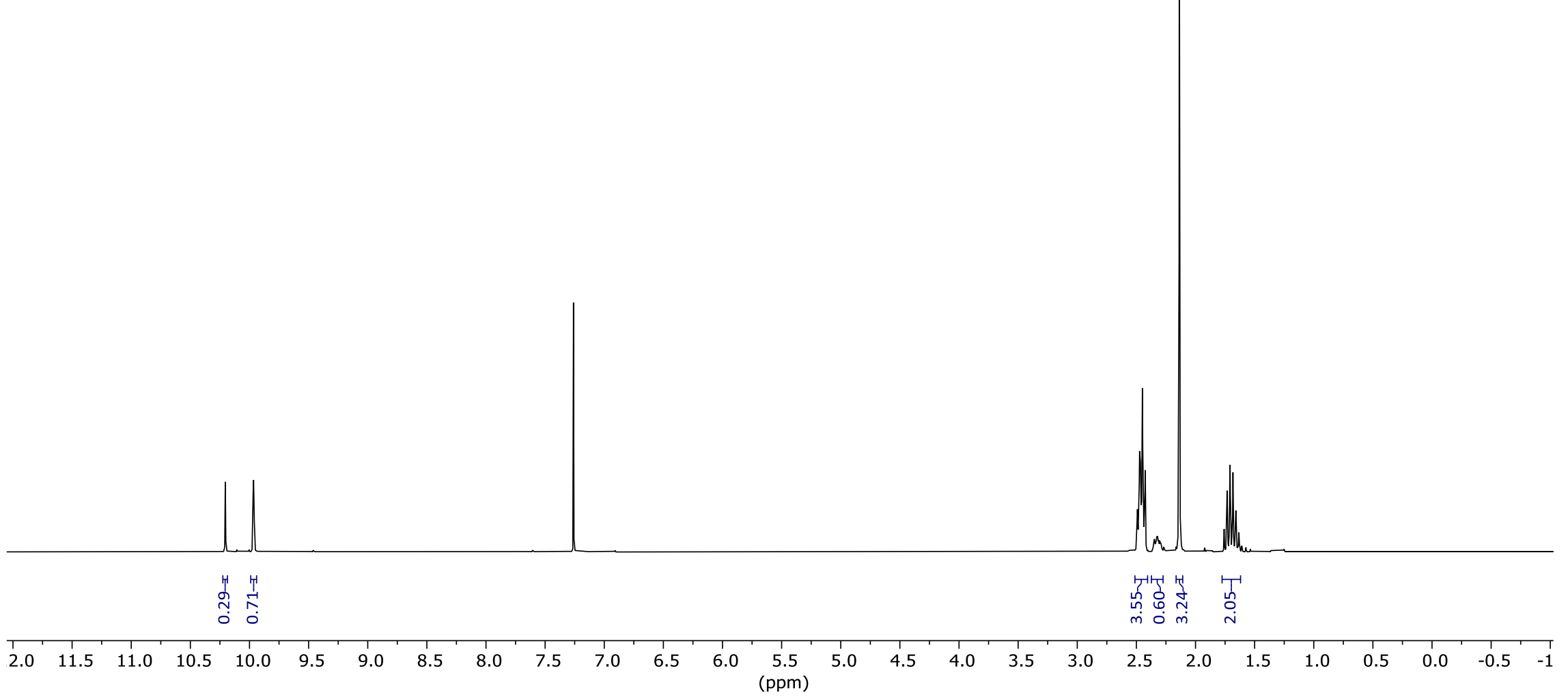


$13 \mathrm{C}\{1 \mathrm{H}\} \mathrm{NMR}, \mathrm{CDCl} 3,75 \mathrm{MHz}$
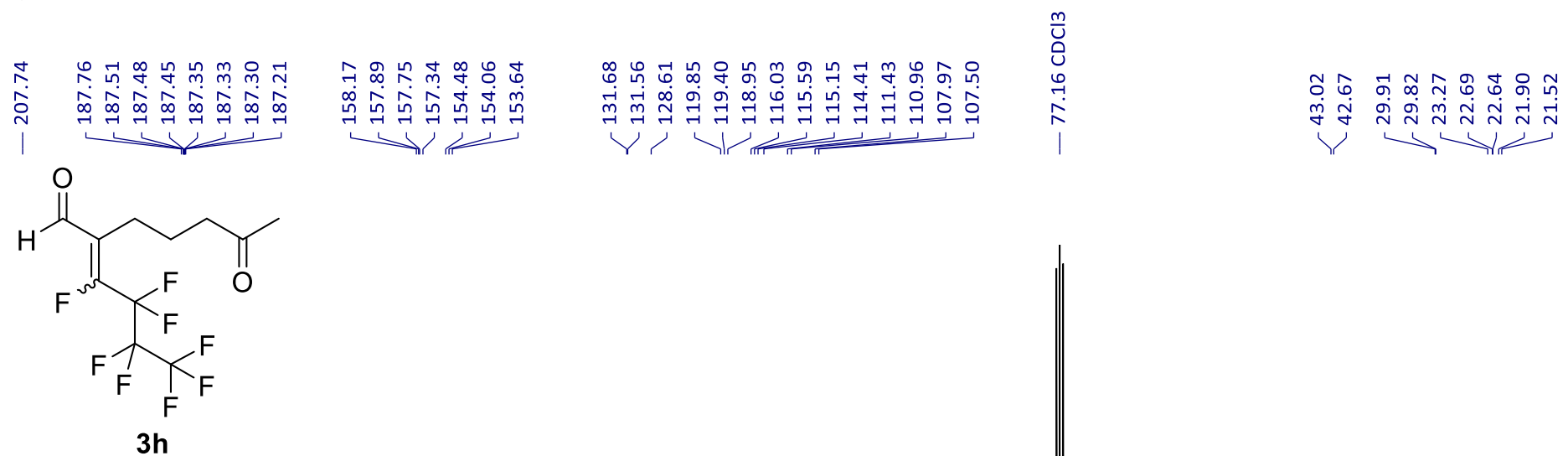

ช่ จ்ุ

$\lambda \sqrt{2}$

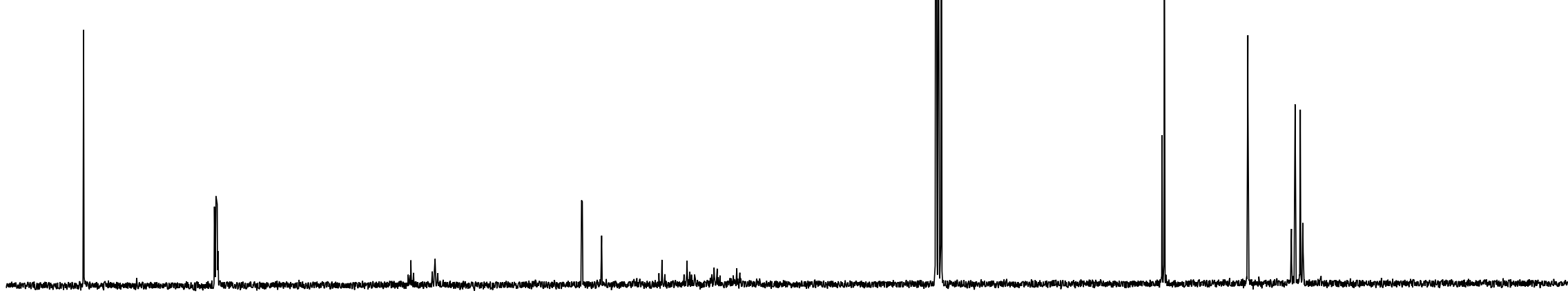

$210 \quad 200$

$\begin{array}{lll}180 & 170\end{array}$

160

140

$130 \quad 120$

$10 \quad 100$

80

70

$60 \quad 50$

40

20 


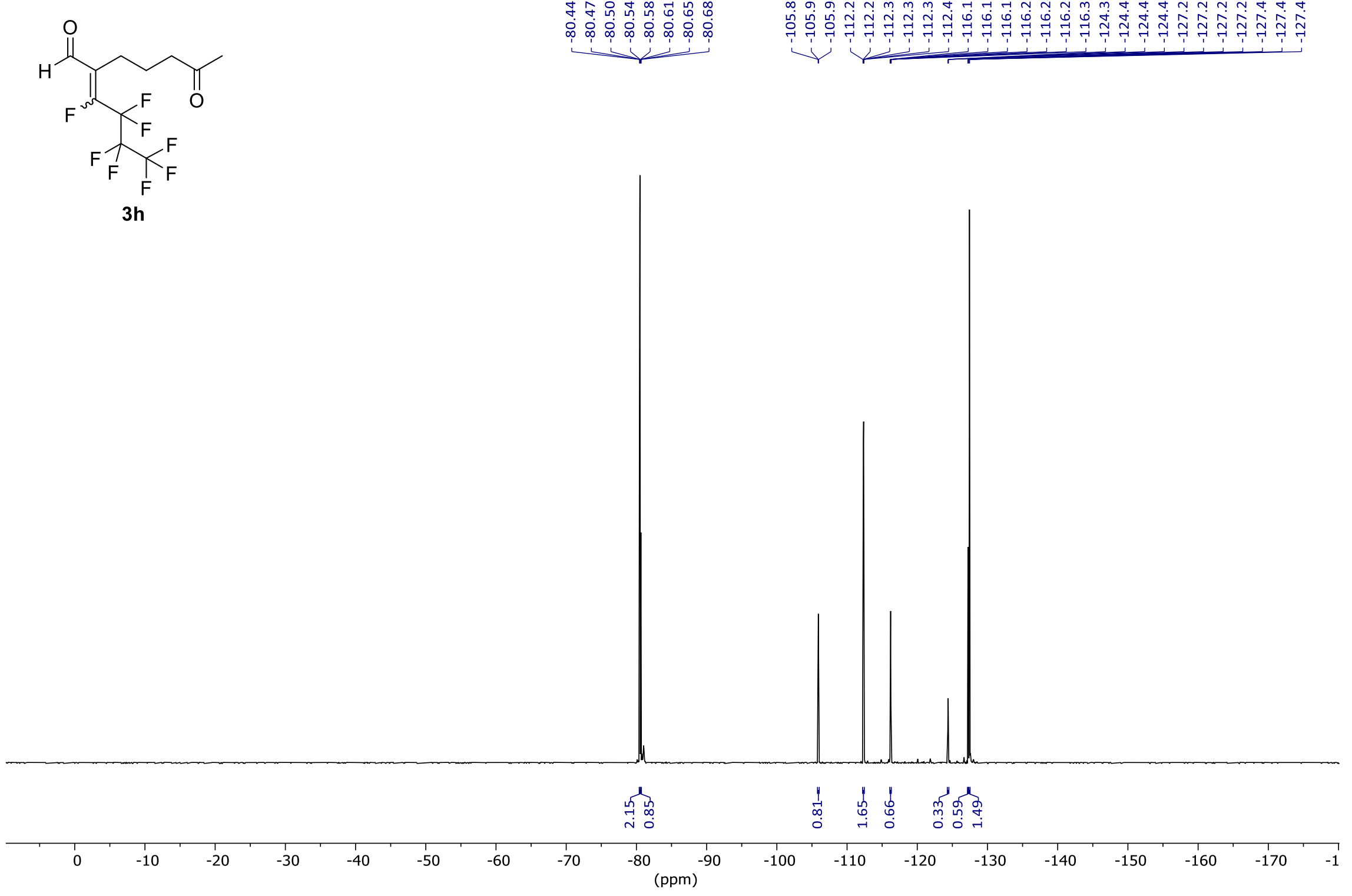


1H NMR, CDCl3, $300 \mathrm{MHz}$

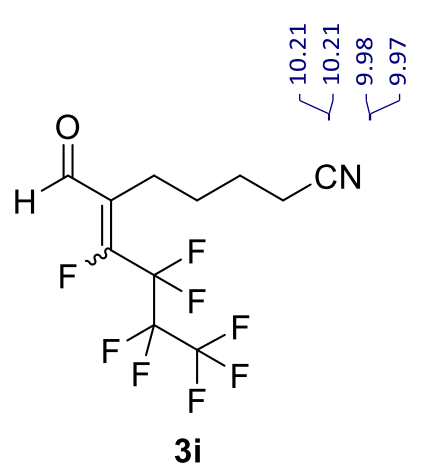

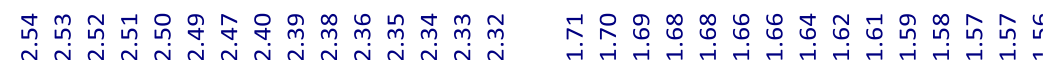

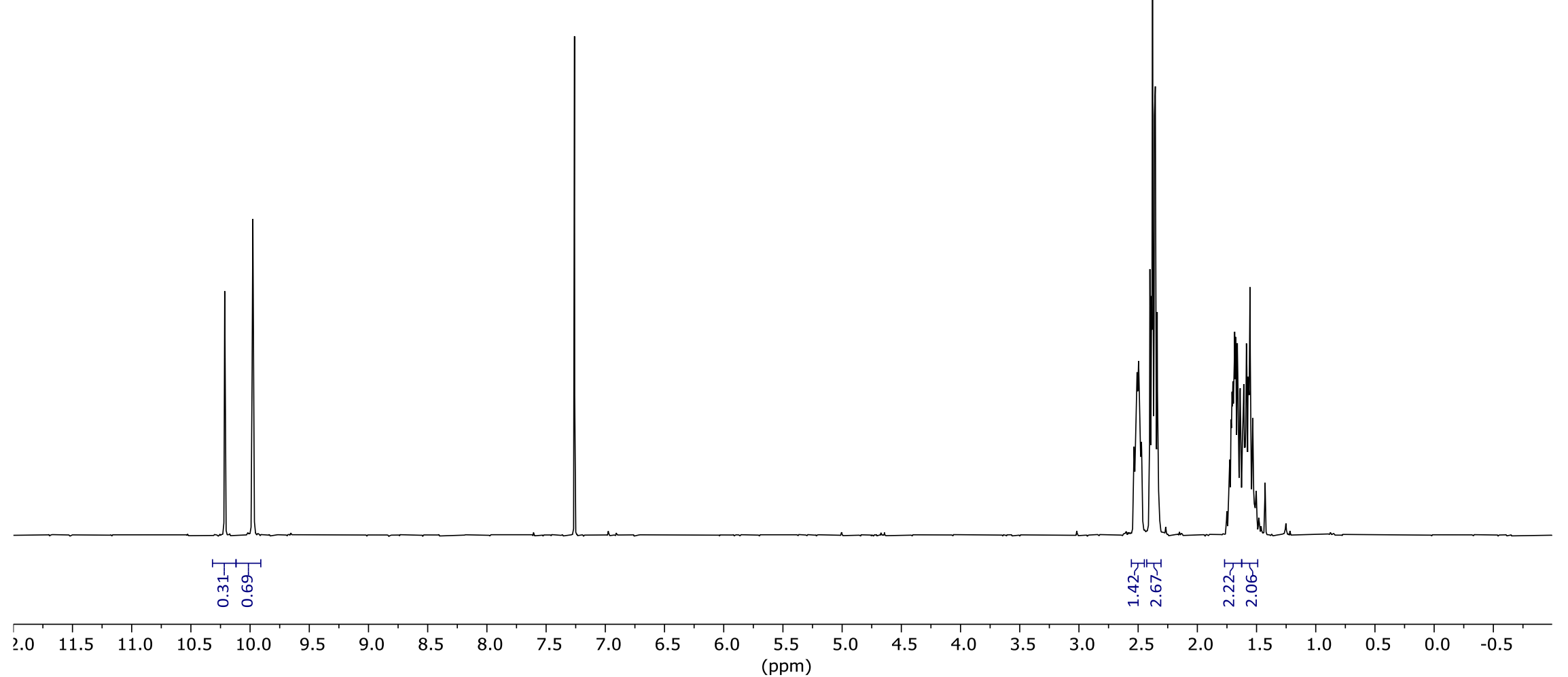


$13 \mathrm{C}\{1 \mathrm{H}\} \mathrm{NMR}, \mathrm{CDCl} 3,75 \mathrm{MHz}$
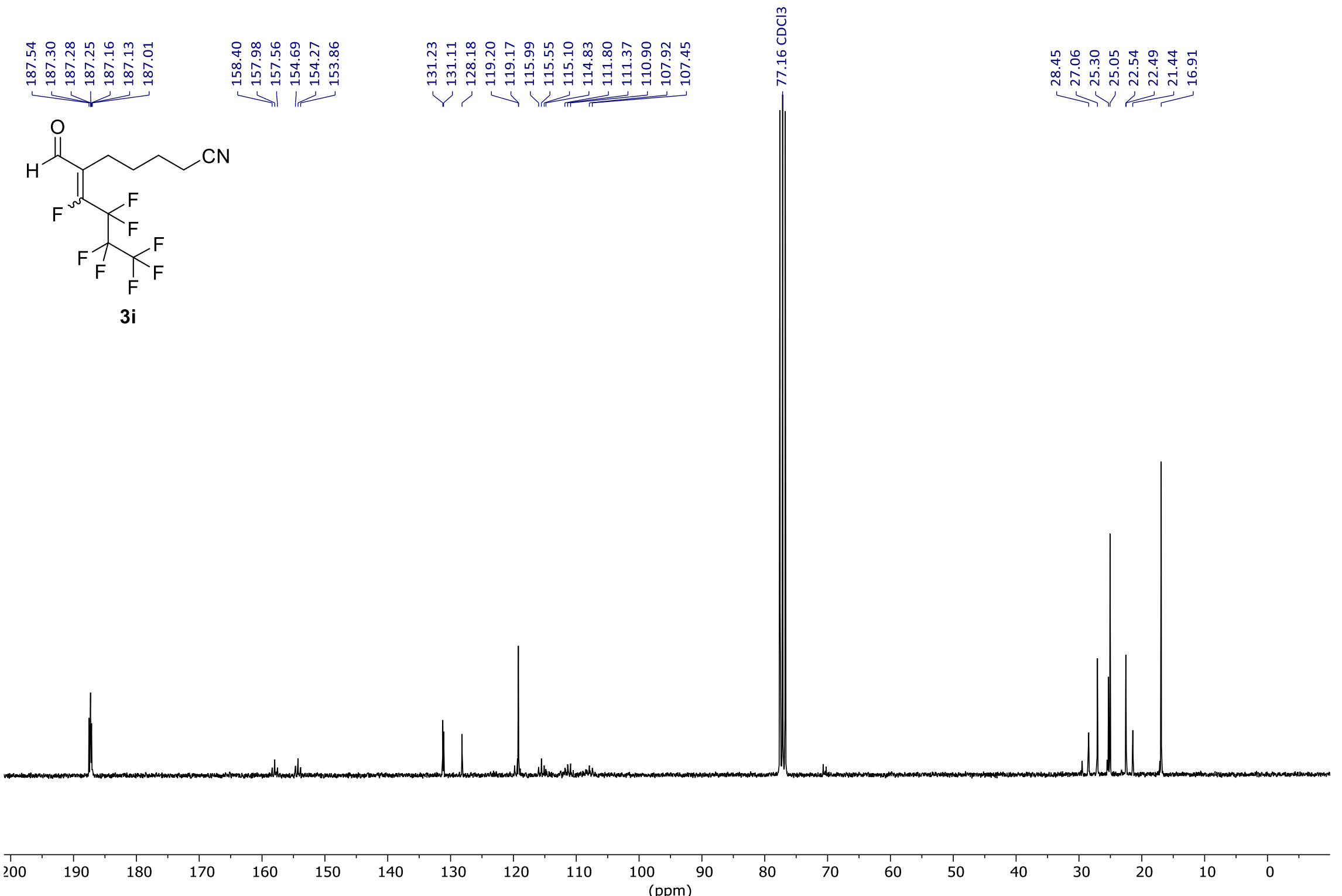
19F NMR, CDCl3, $282 \mathrm{MHz}$

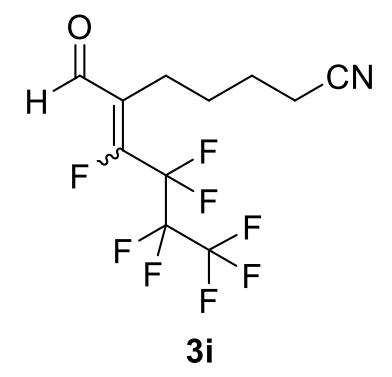

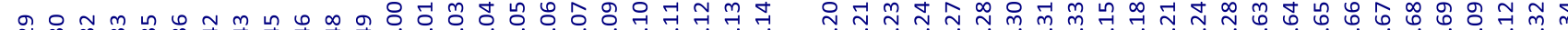

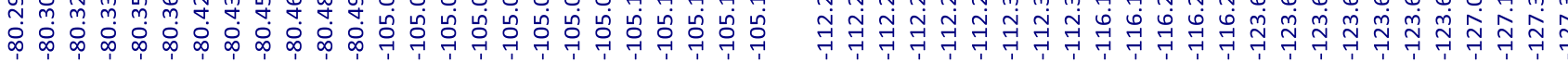

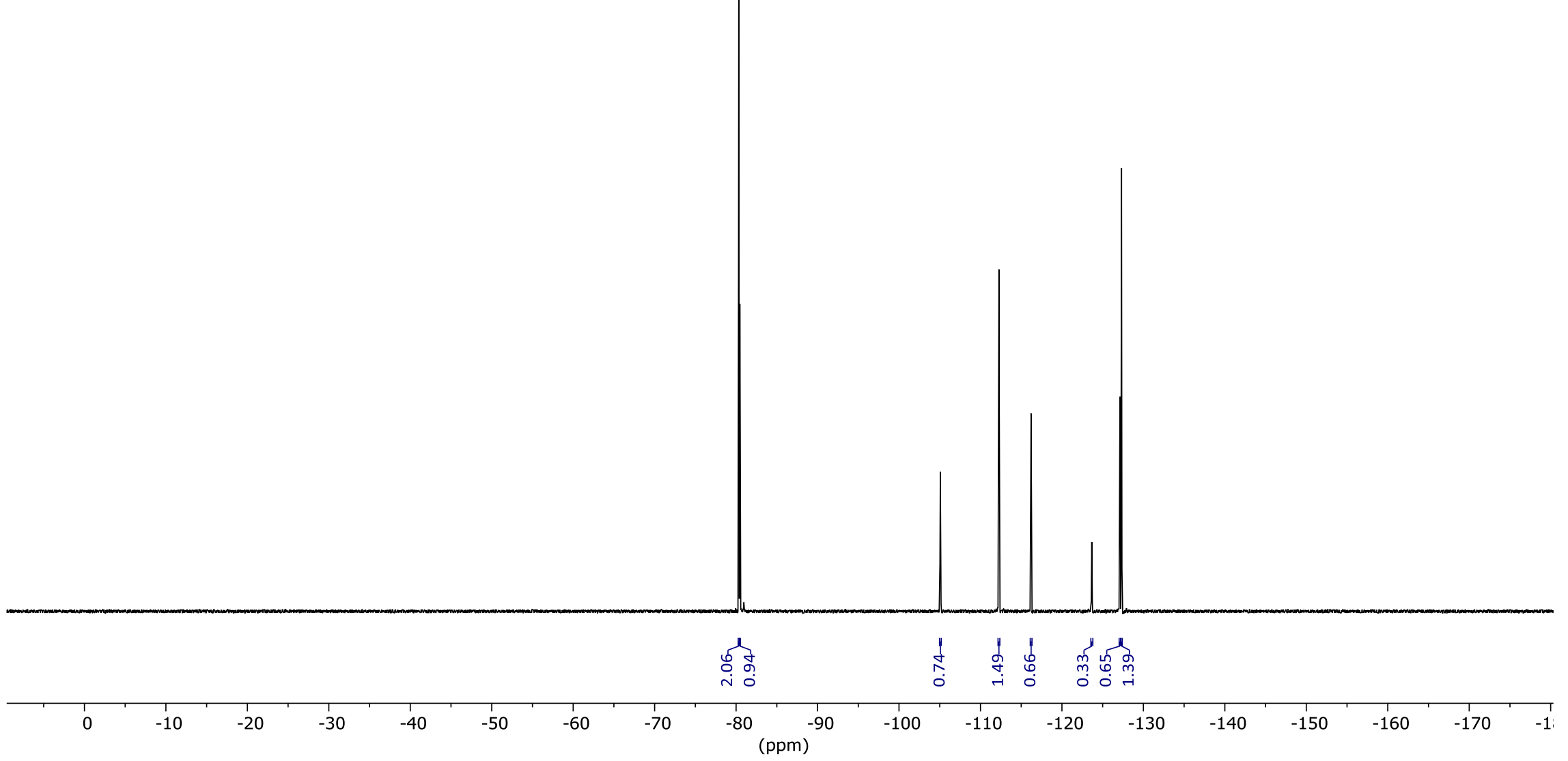


1H NMR, CDCl3, $300 \mathrm{MHz}$

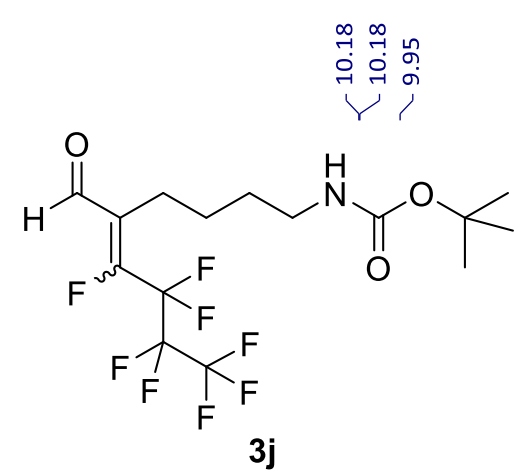

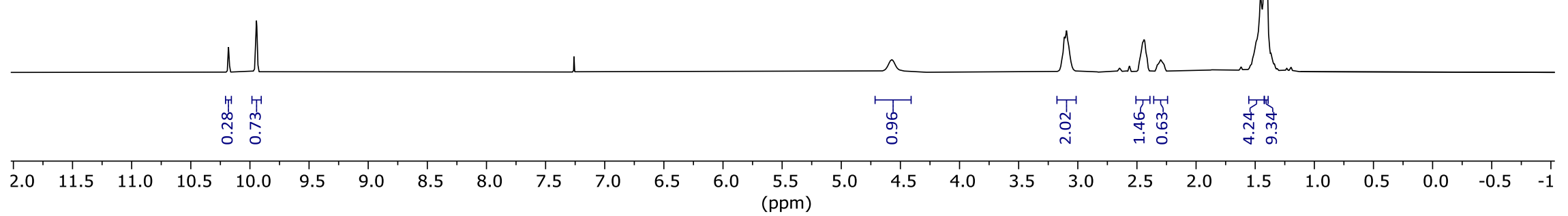


$13 \mathrm{C}\{1 \mathrm{H}\} \mathrm{NMR}, \mathrm{CDCl} 3,75 \mathrm{MHz}$

$\underbrace{-1}$
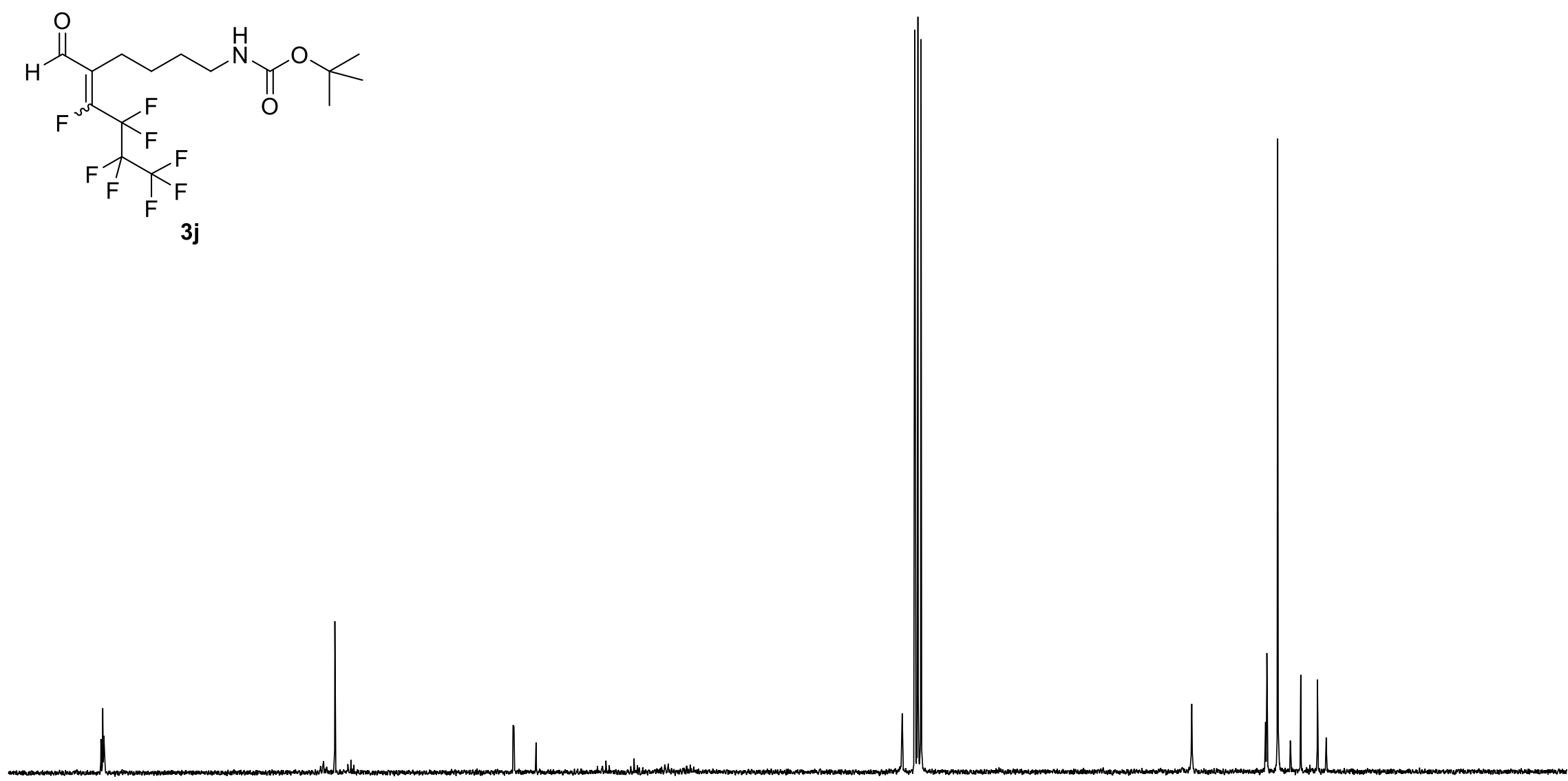

160 
19F NMR, CDCl3, $282 \mathrm{MHz}$

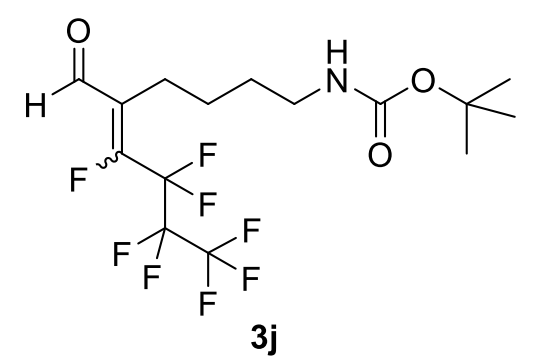

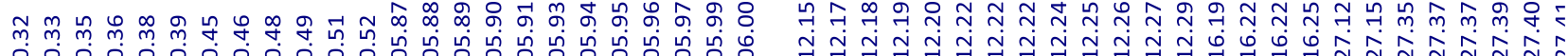

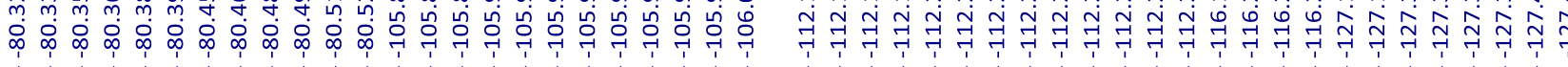

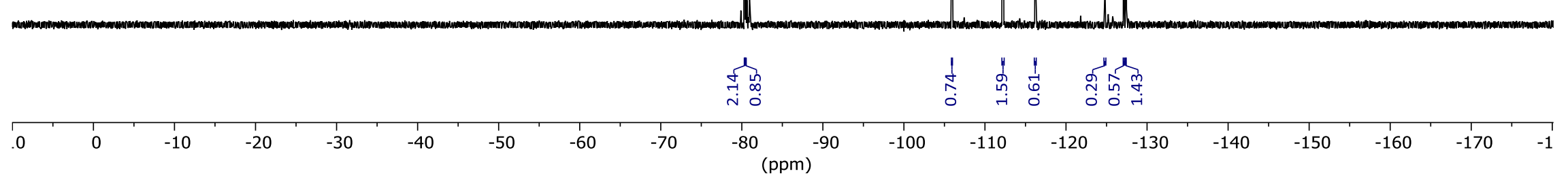


1H NMR, CDCl3, $300 \mathrm{MHz}$

范

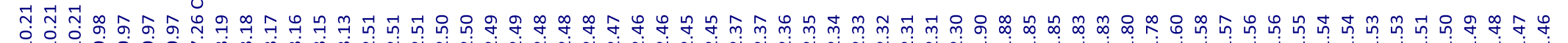

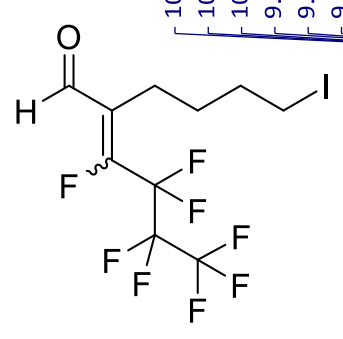

$3 \mathbf{k}$

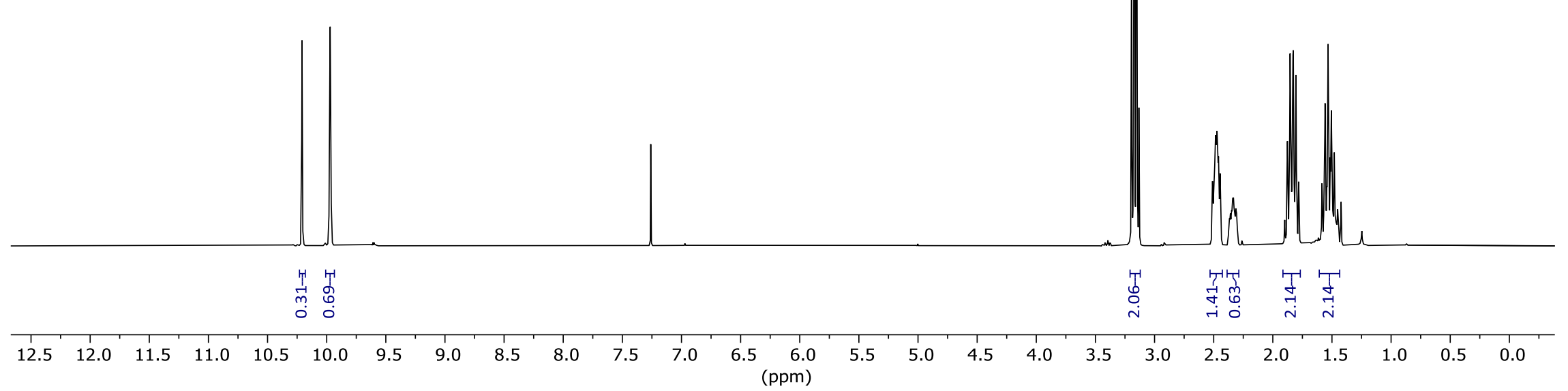


$13 \mathrm{C}\{1 \mathrm{H}\} \mathrm{NMR}, \mathrm{CDCl} 3,75 \mathrm{MHz}$

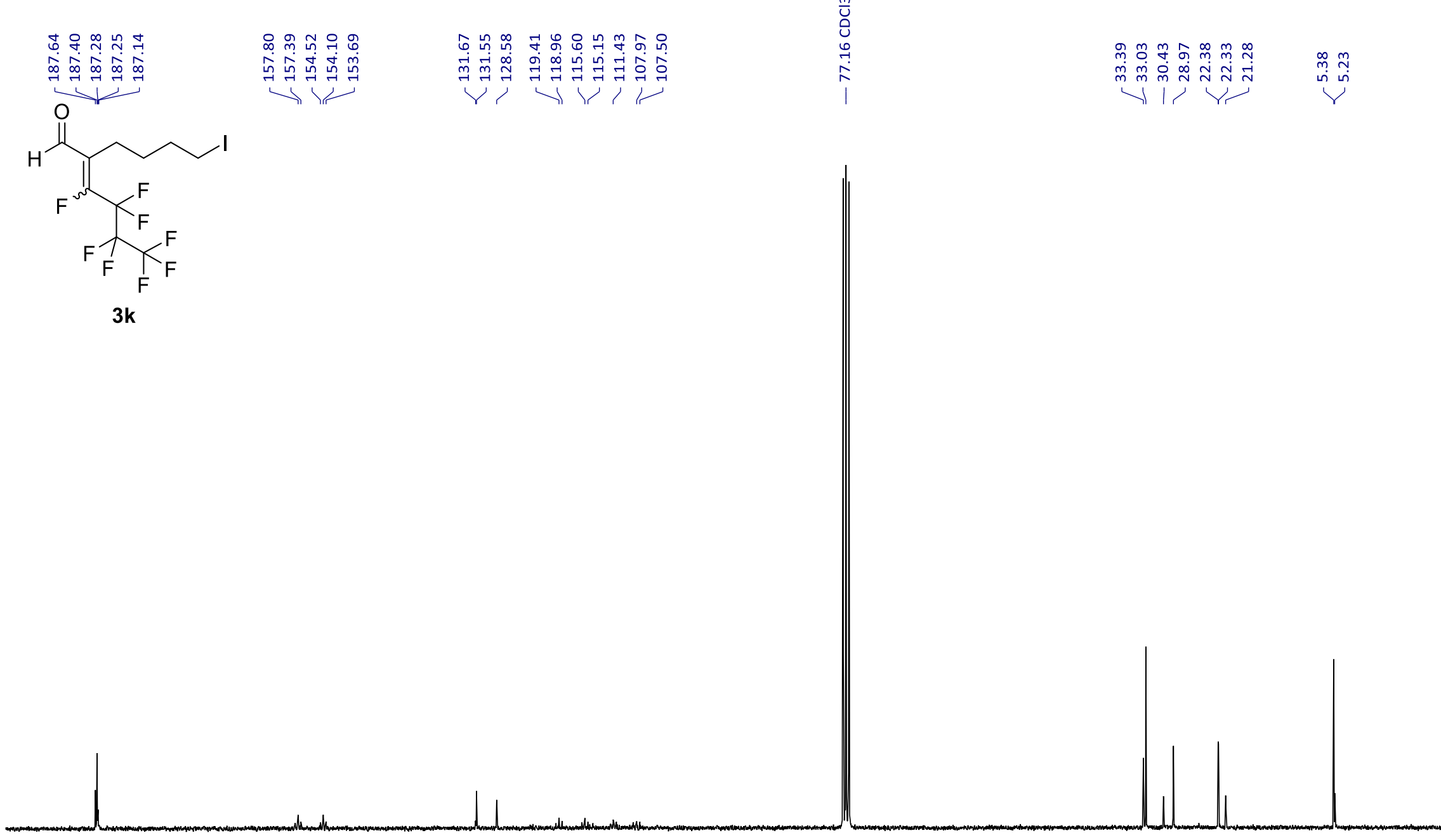

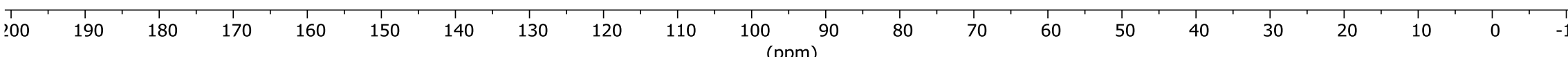




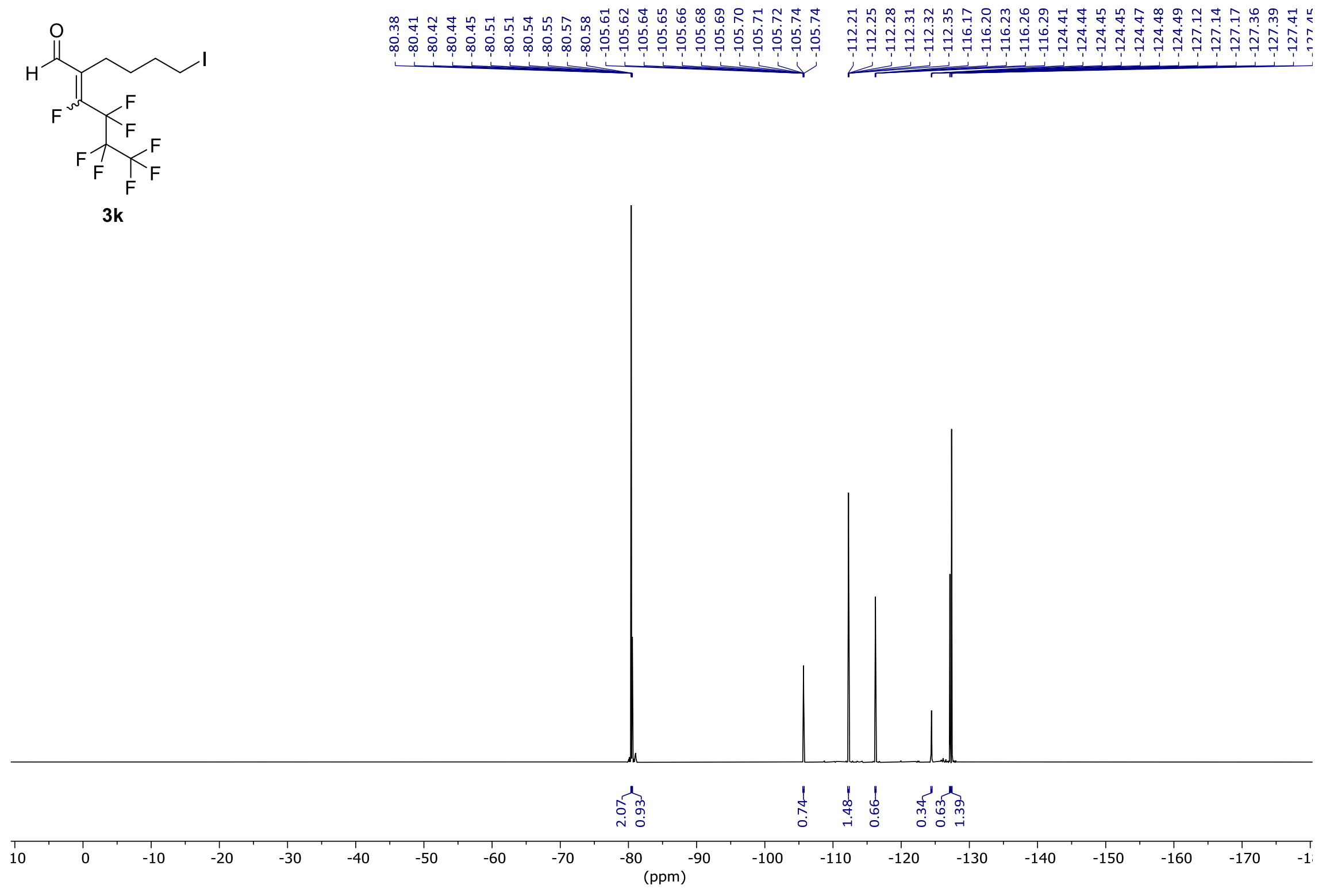


$1 \mathrm{H} \mathrm{NMR}, \mathrm{CDCl} 3,300 \mathrm{MHz}$

兽

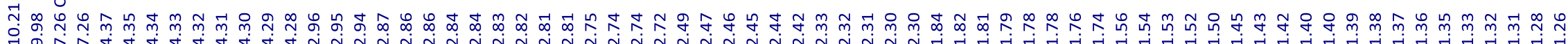
烈

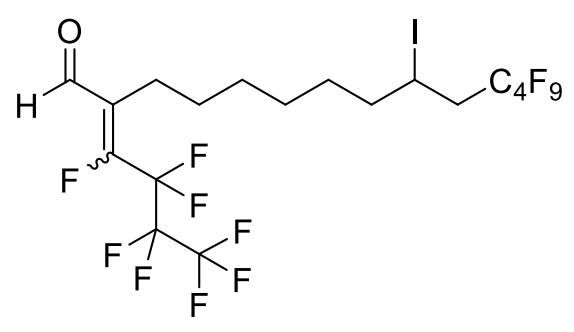

31

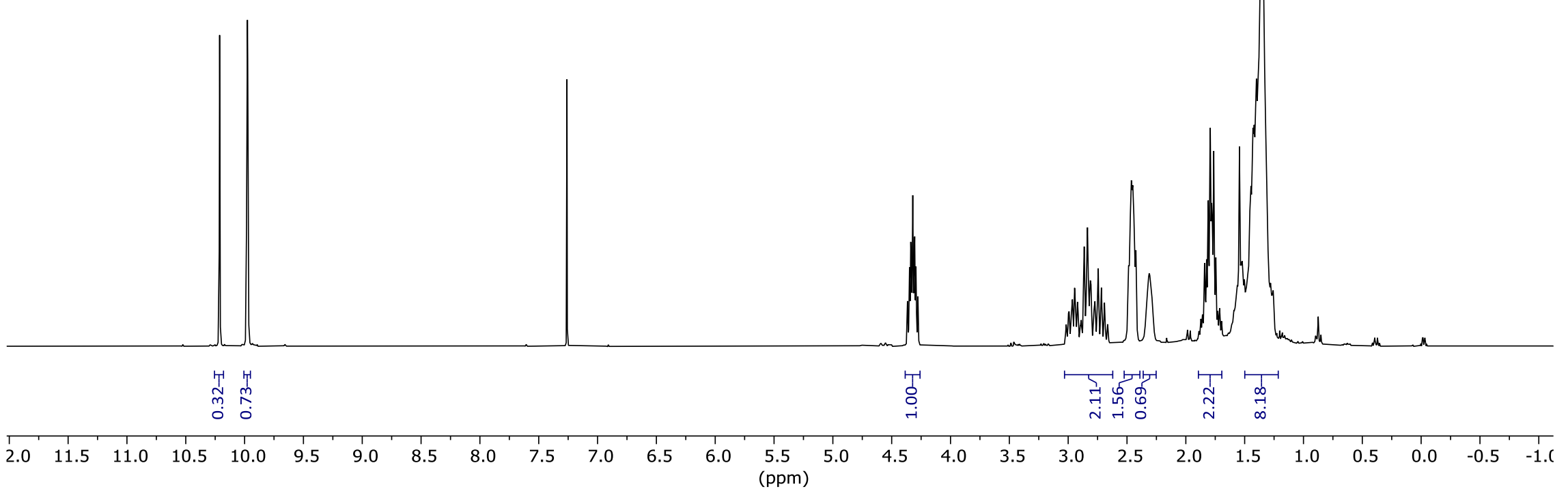


$13 \mathrm{C}\{1 \mathrm{H}\} \mathrm{NMR}, \mathrm{CDCl} 3,75 \mathrm{MHz}$

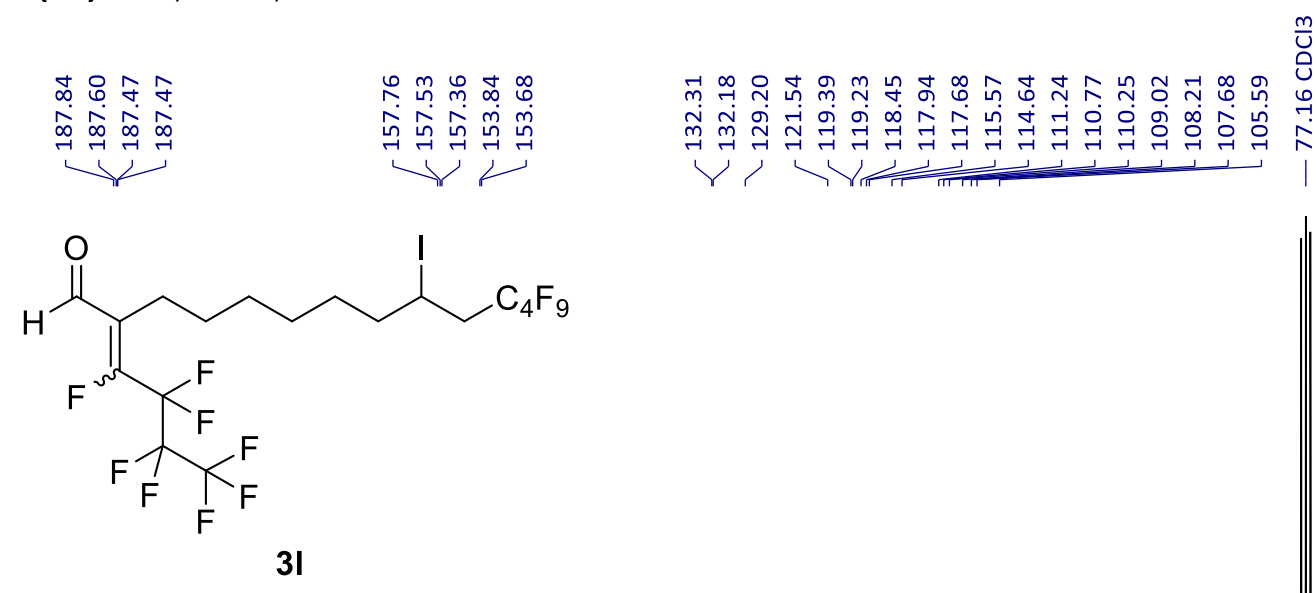

๓ กำ

守

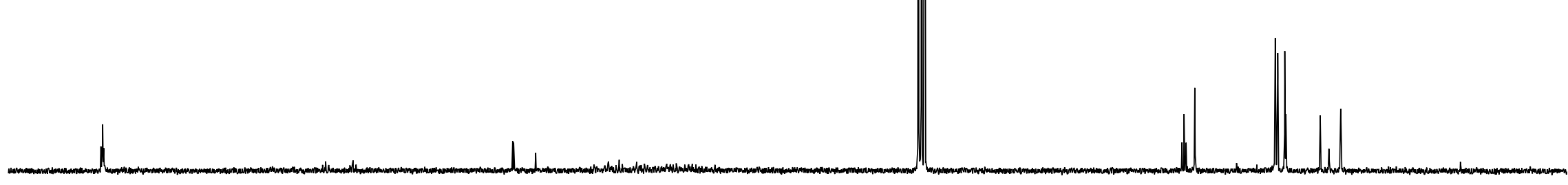

\begin{tabular}{rrr}
\hline & 190 & 180
\end{tabular} 
19F NMR, $\mathrm{CDCl} 3,282 \mathrm{MHz}$

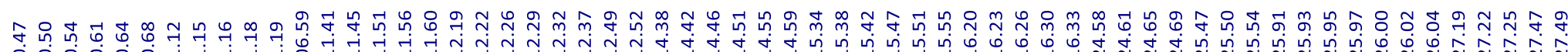

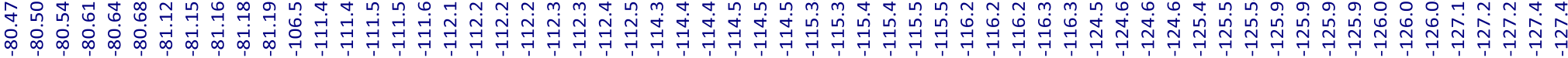<smiles>O=CC(CCCCCCC(I)CC(F)(F)F)C(F)(F)C(F)(F)C(F)(F)F</smiles>

\section{1}

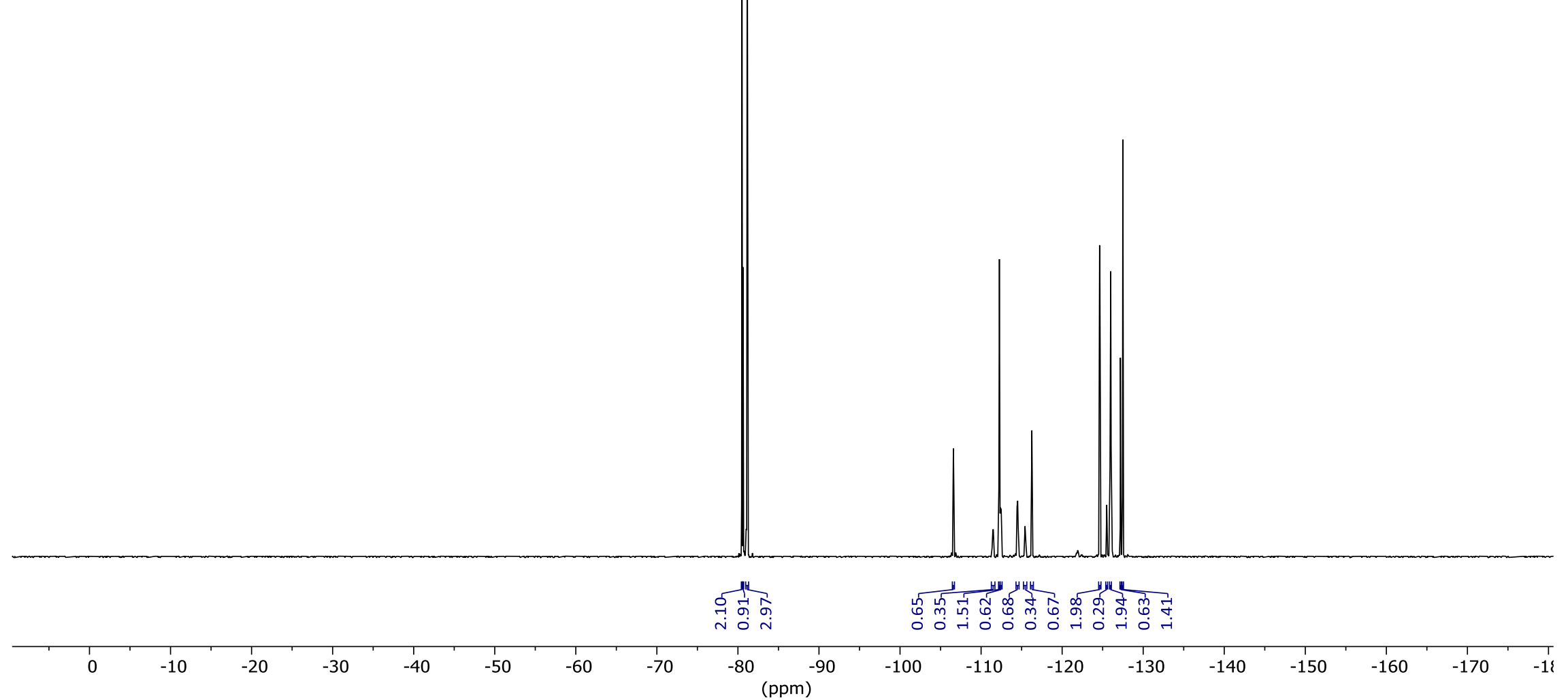




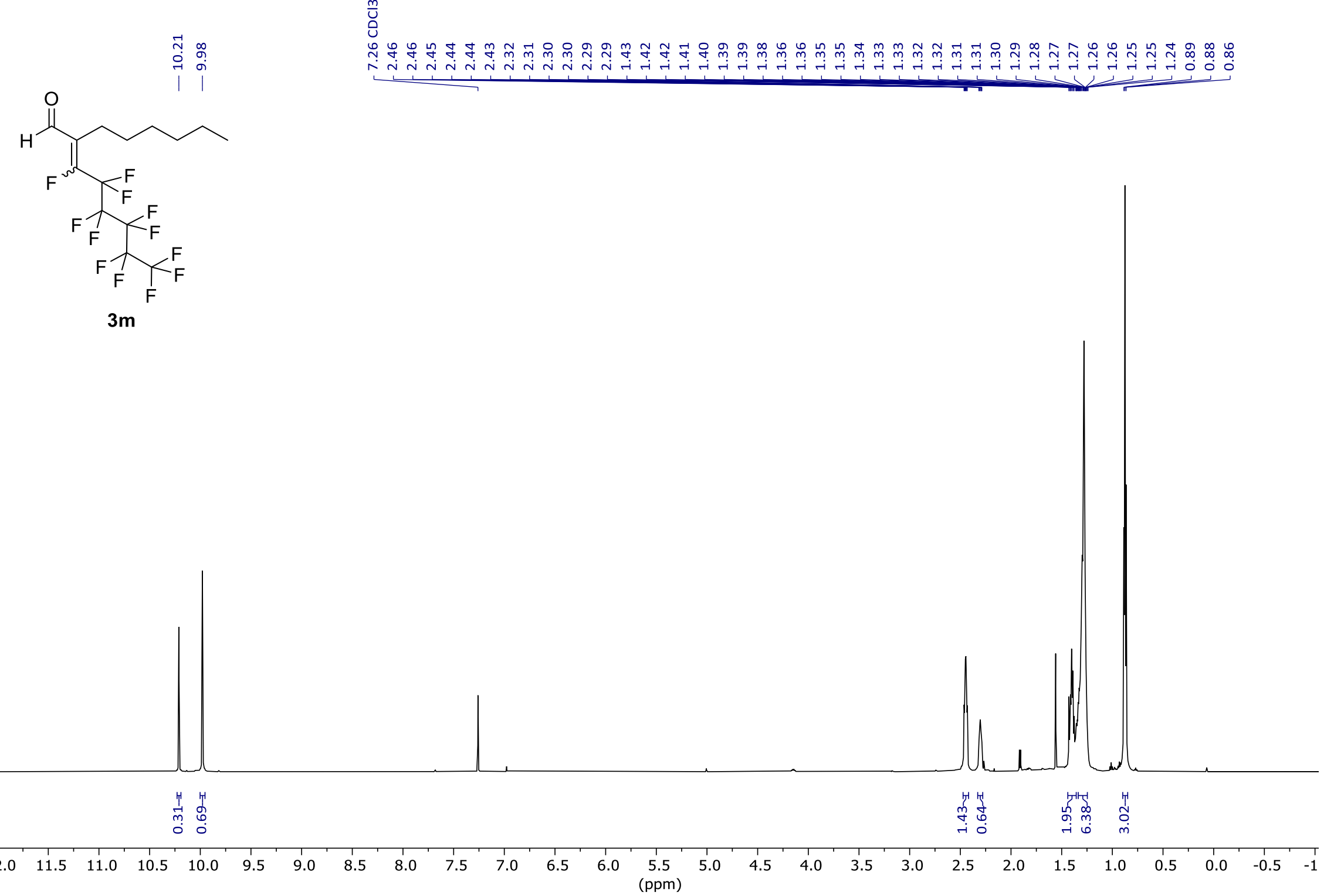


ơ

年

r

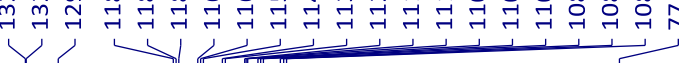

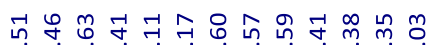

m

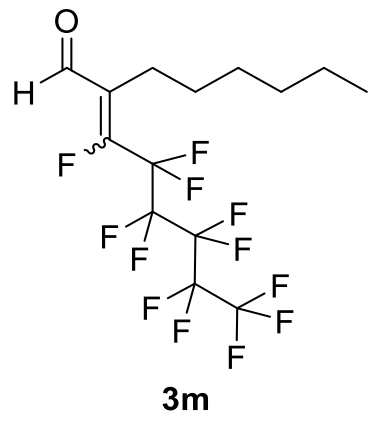

$3 \mathrm{~m}$

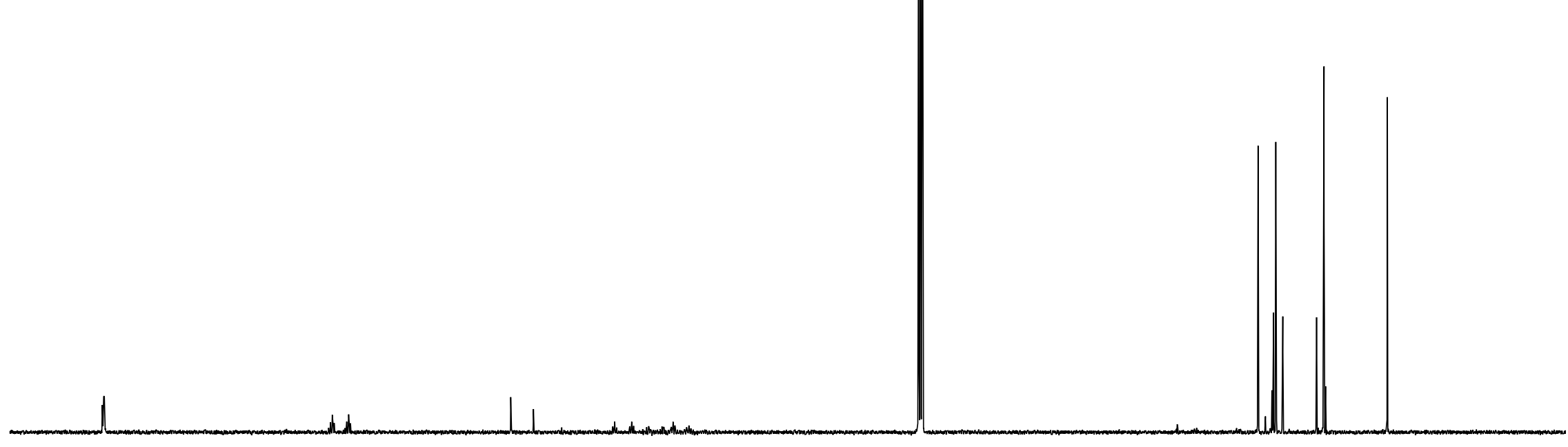

$00 \quad 190$ 
19F NMR, CDCl3, $565 \mathrm{MHz}$

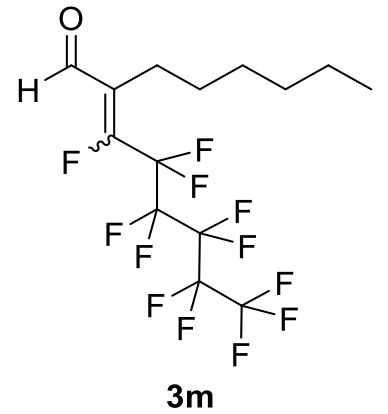

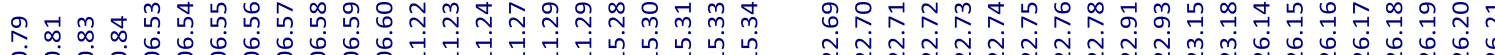

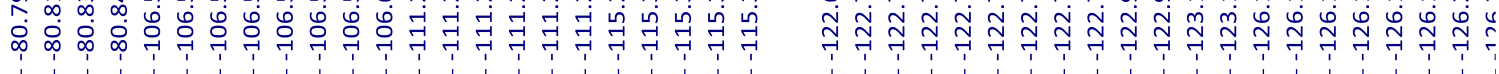


1H NMR, CDCl3, $300 \mathrm{MHz}$

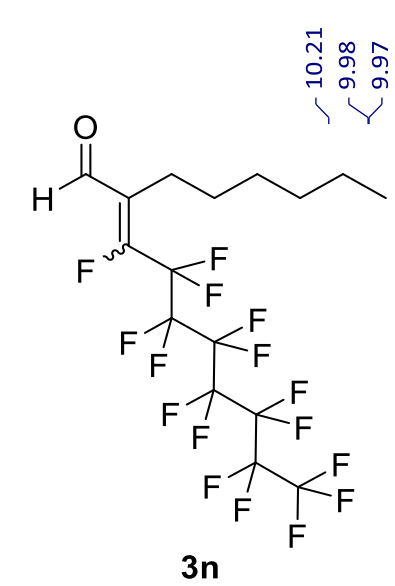

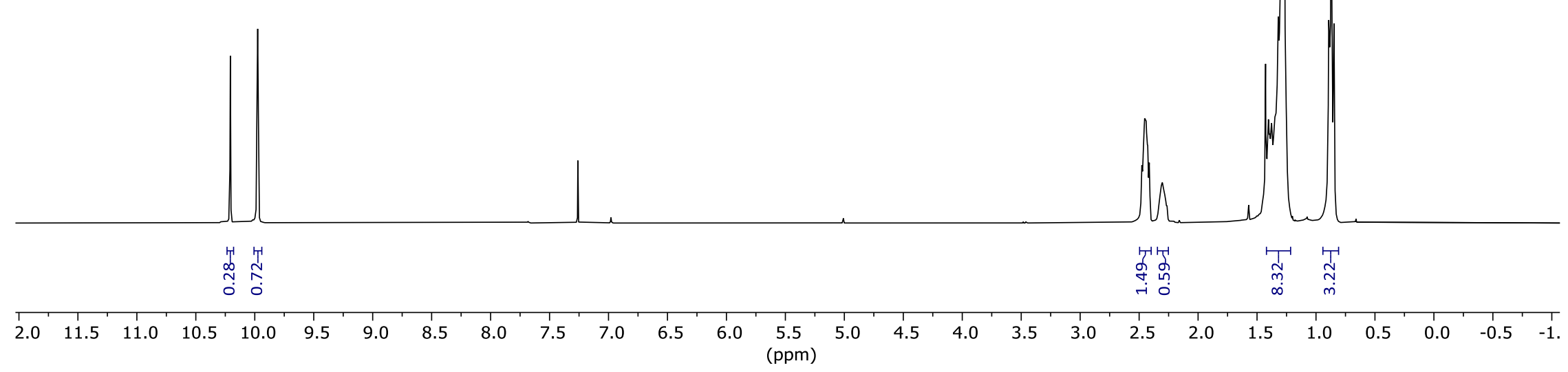



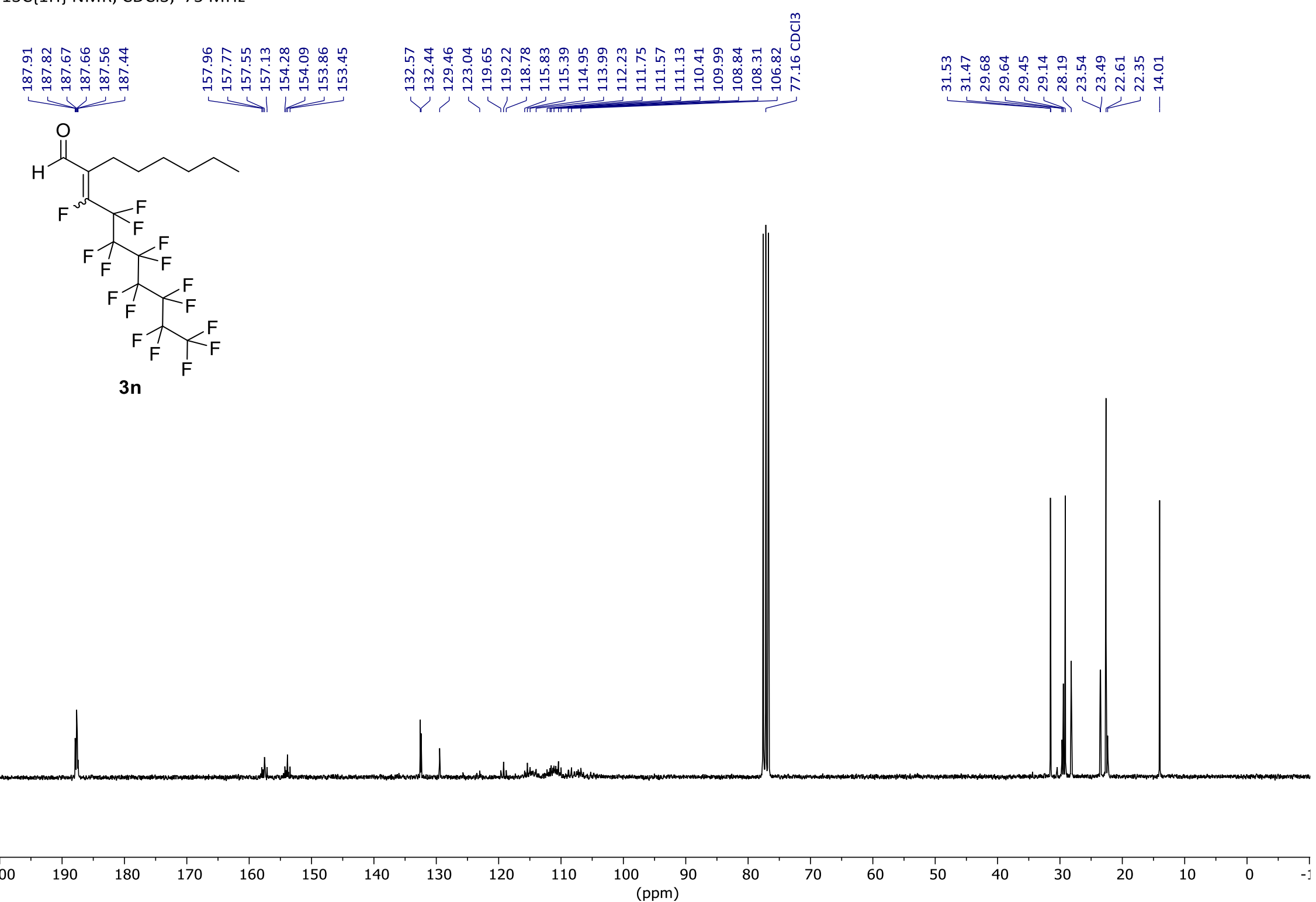
19F NMR, CDCl3, $282 \mathrm{MHz}$

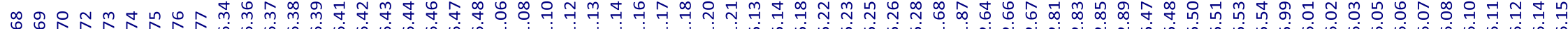

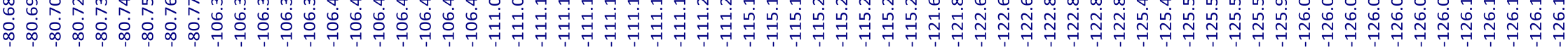

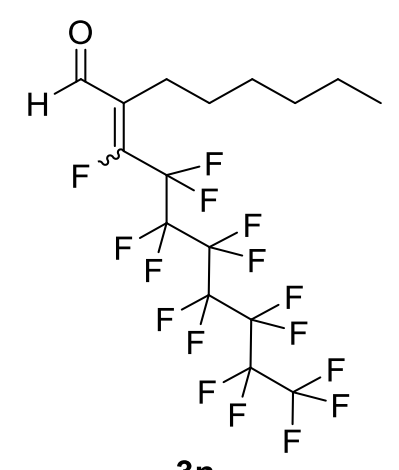

$3 n$

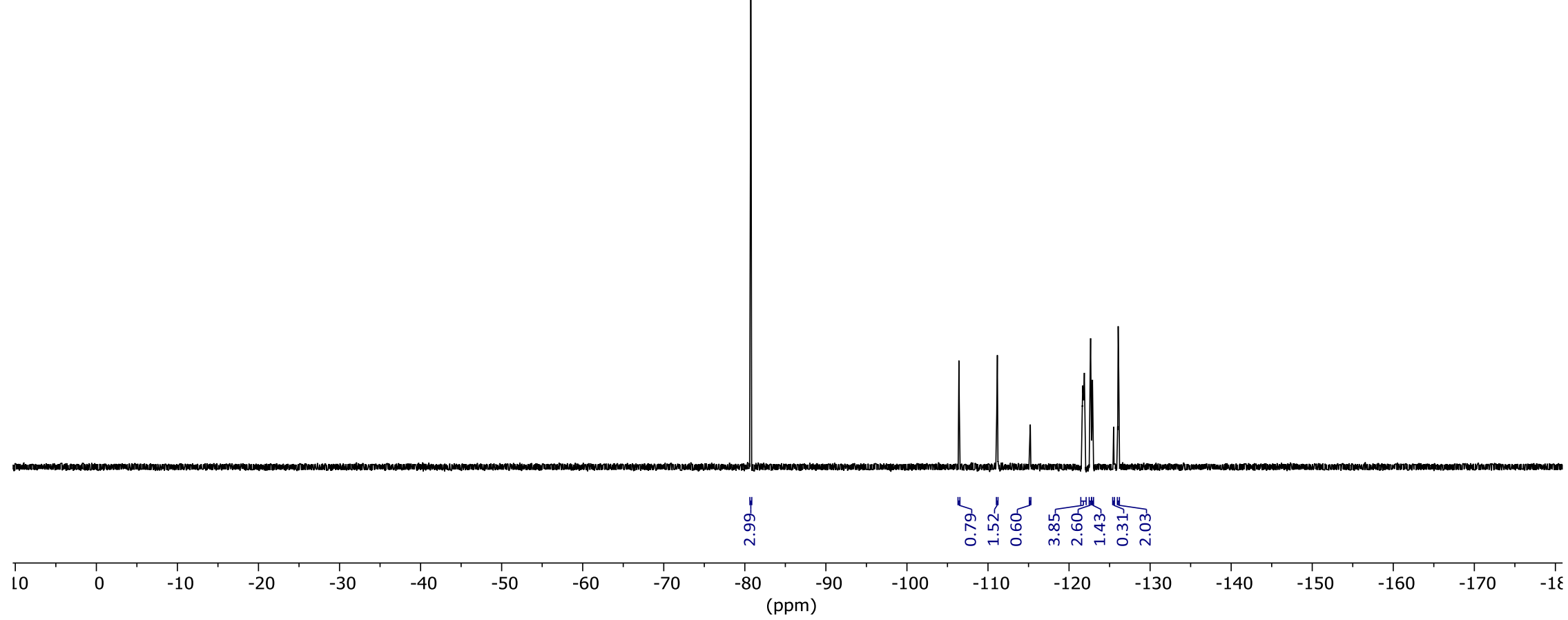


1H NMR, CDCl3, $300 \mathrm{MHz}$

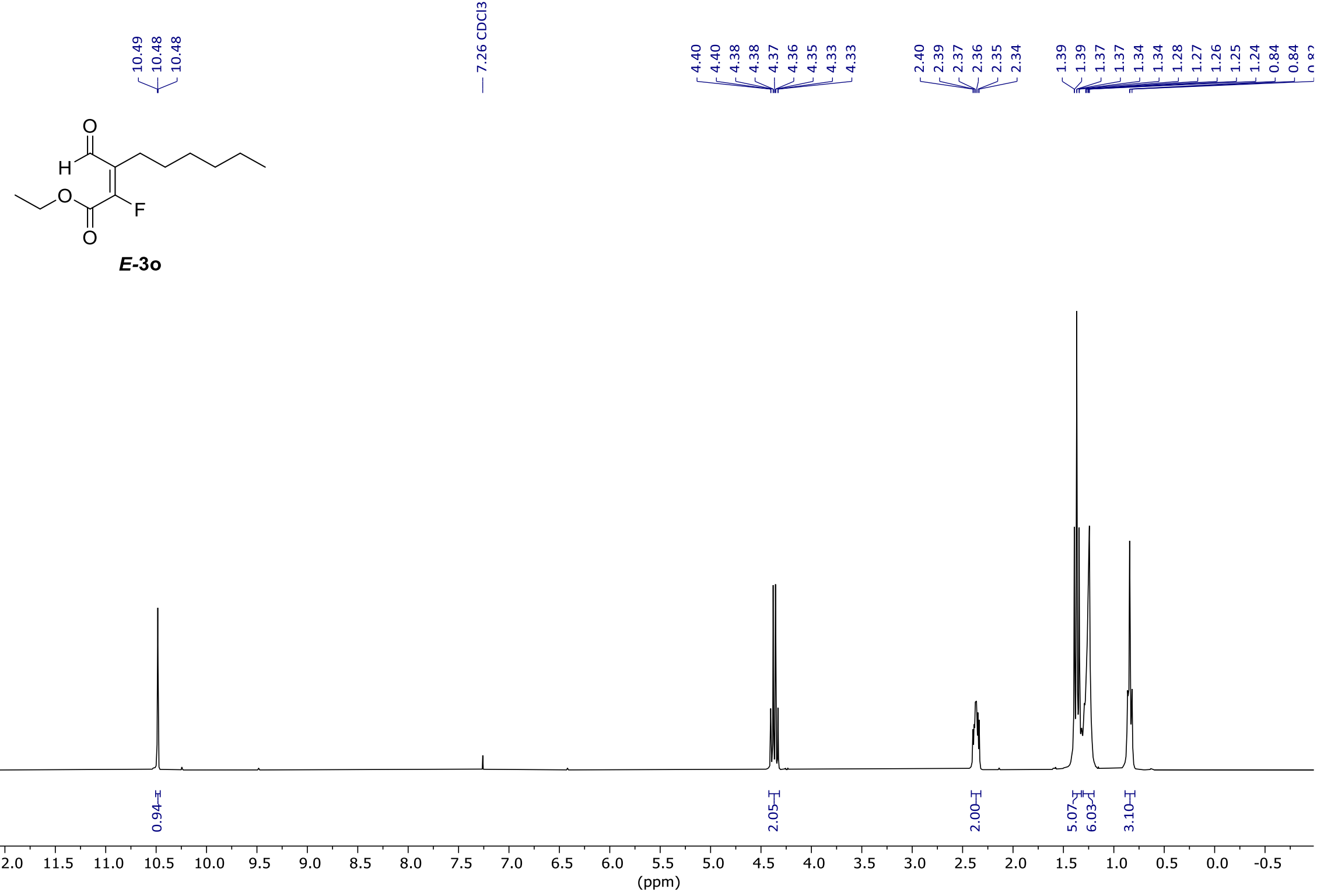


$13 \mathrm{C}\{1 \mathrm{H}\} \mathrm{NMR}, \mathrm{CDCl} 3,126 \mathrm{MHz}$
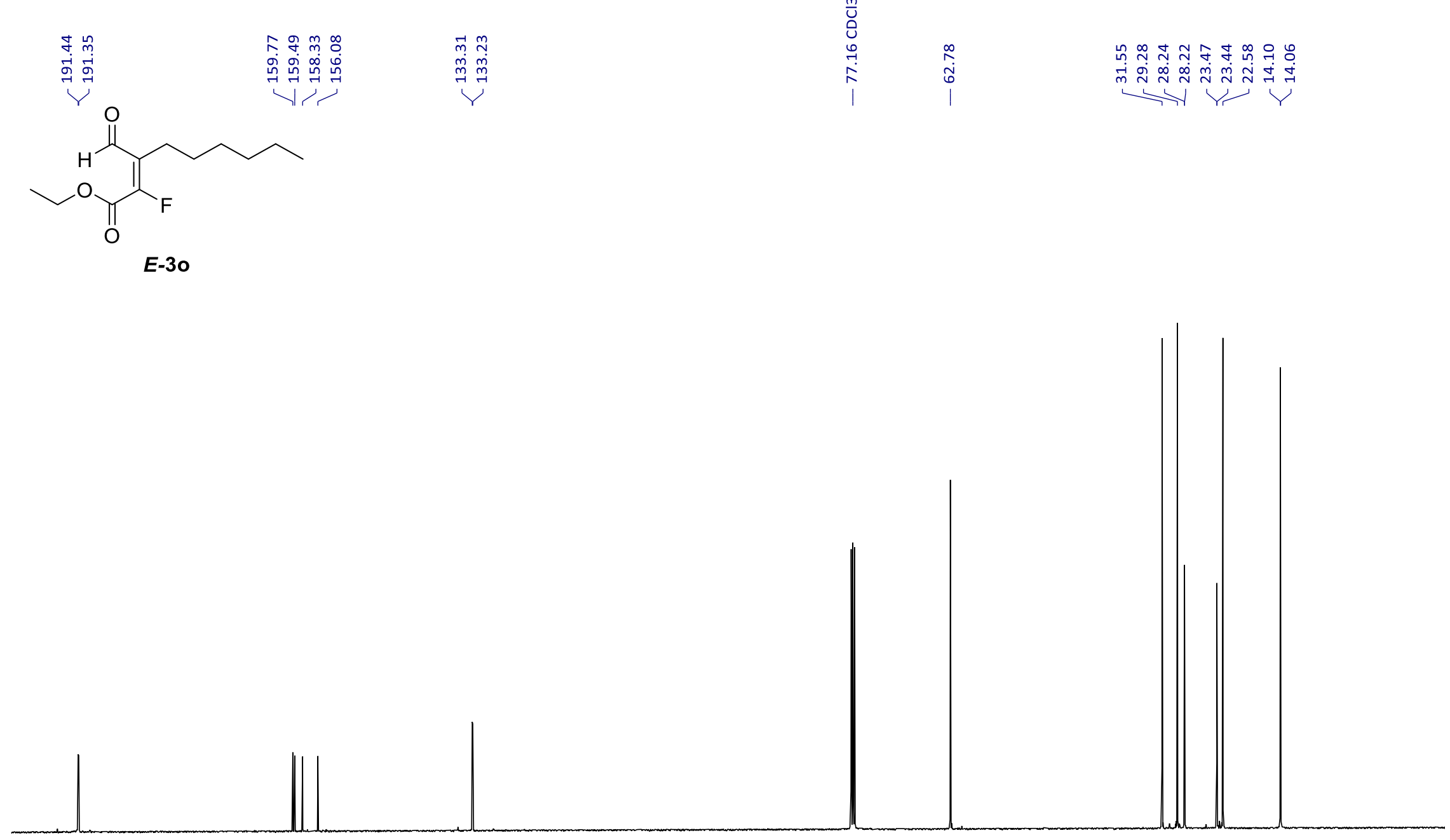

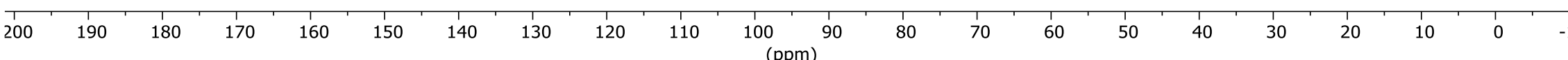


19F NMR, CDCl3, $282 \mathrm{MHz}$

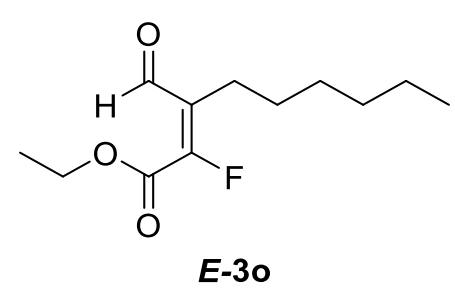

$\begin{array}{ll}20 & 0 \\ 0 & 0 \\ \dot{0} & 0 \\ 0 & 7 \\ & 1\end{array}$

E-30

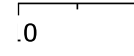

$-10 \quad-20$

$-30$

$-40 \quad-50$

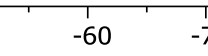

$-70 \quad-80$ (ppm) 
1H NMR, CDCl3, $300 \mathrm{MHz}$

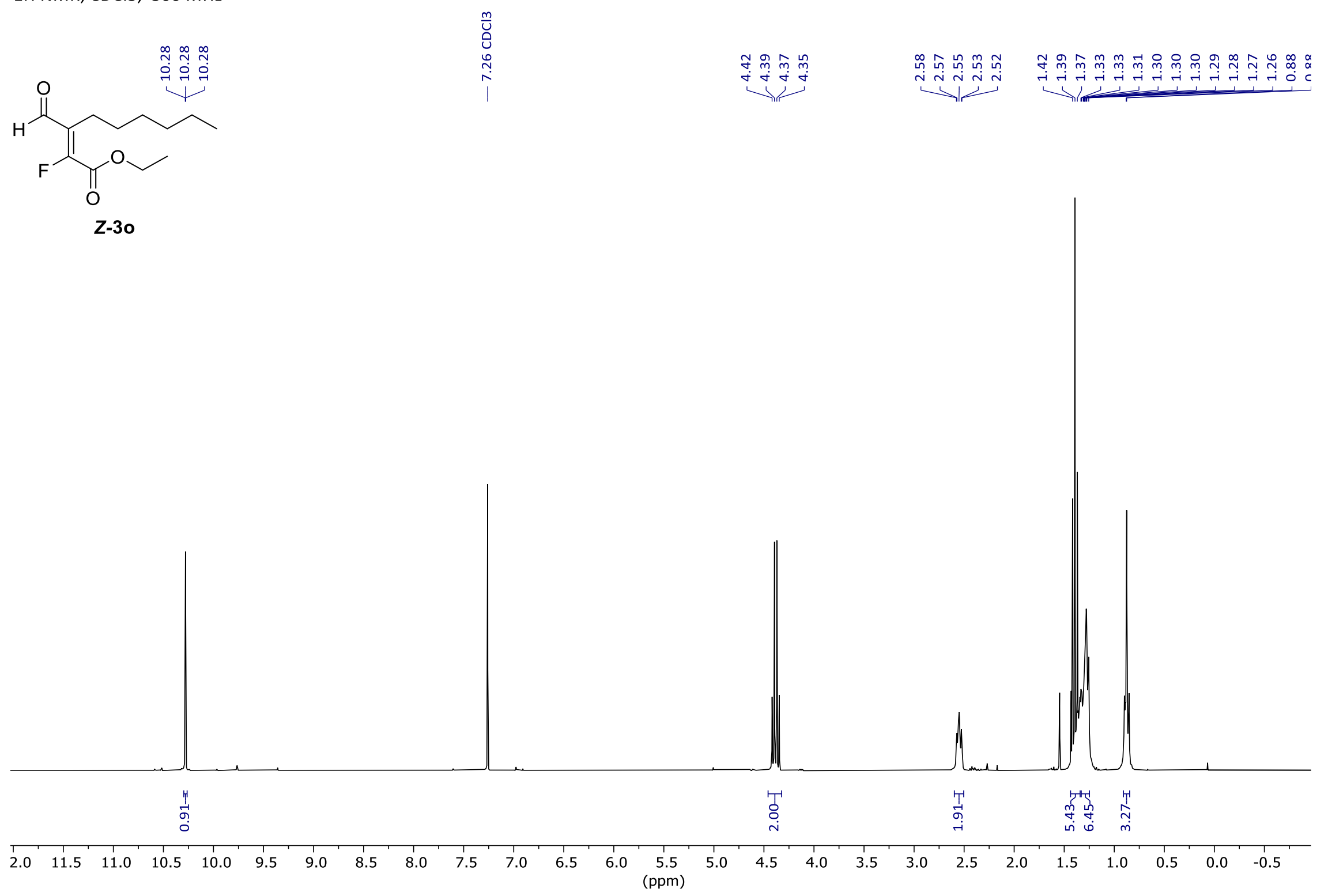


$13 \mathrm{C}\{1 \mathrm{H}\} \mathrm{NMR}, \mathrm{CDCl} 3,126 \mathrm{MHz}$
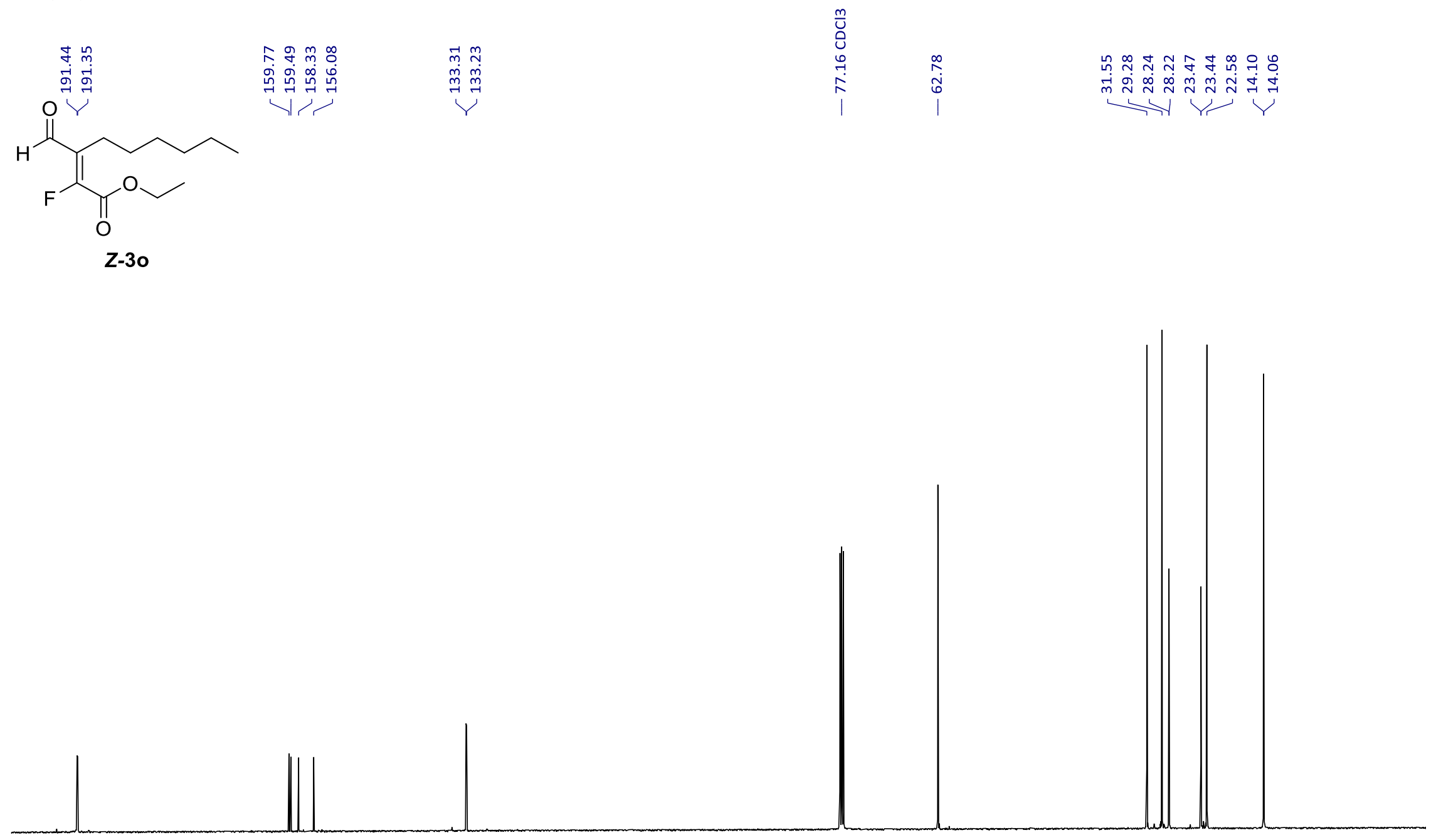

200

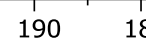

$80 \quad 170$

150

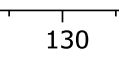

$100 \quad 90$

(ppm) 
19F NMR, $\mathrm{CDCl} 3,282 \mathrm{MHz}$<smiles>CCCCCC/C(C(=O)OCC)=C(\F)C(=O)OCC</smiles>

Z-30

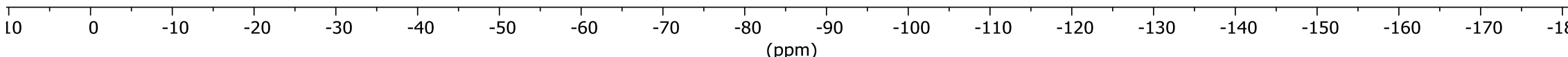




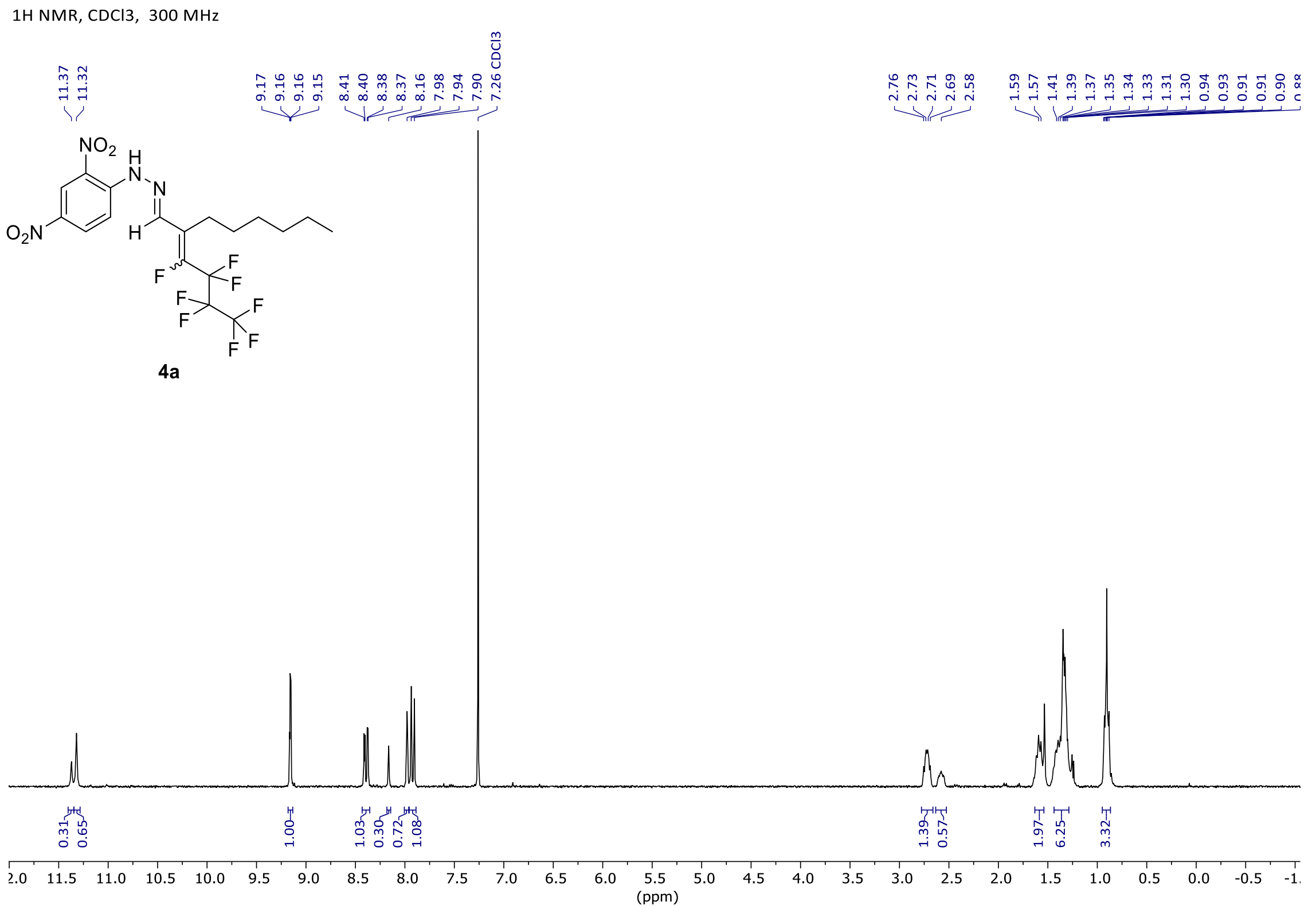



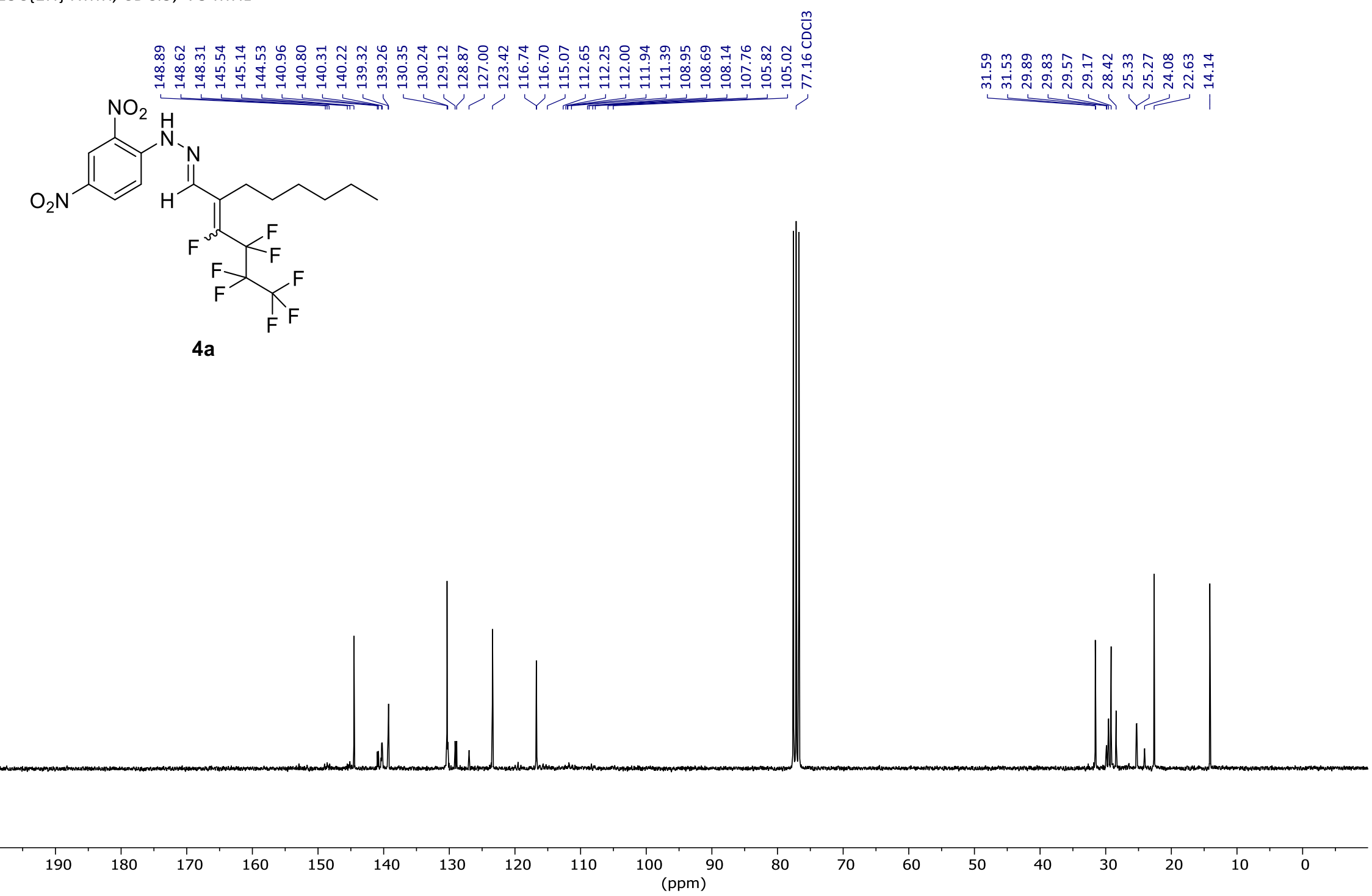
19F NMR, CDCl3, $282 \mathrm{MHz}$

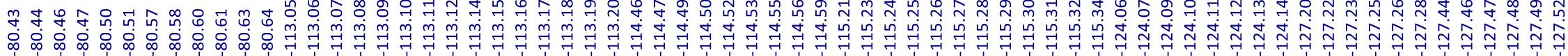
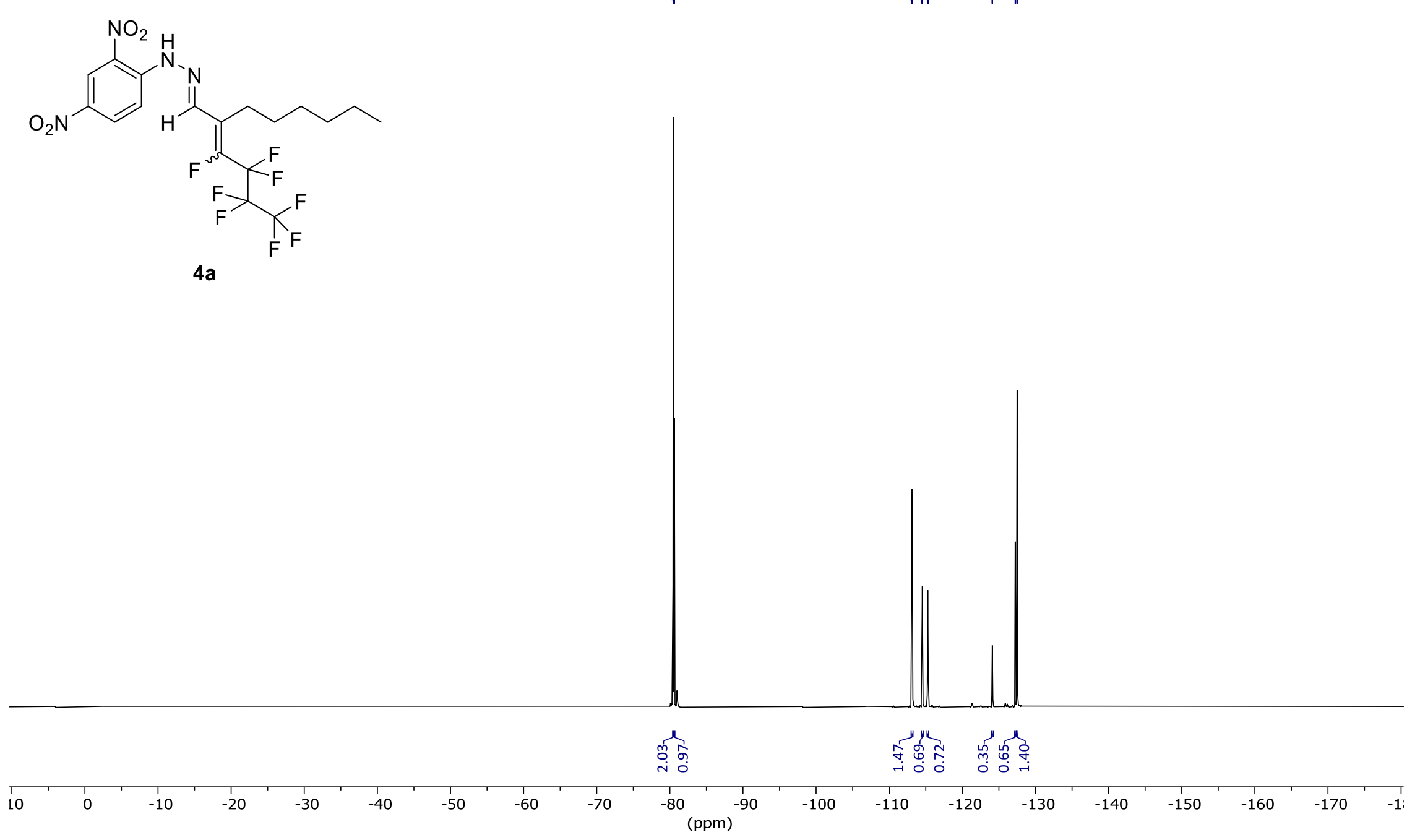
$1 \mathrm{H} \mathrm{NMR,} \mathrm{CDCl} 3,300 \mathrm{MHz}$

$\frac{\mathrm{u}}{\mathrm{u}}$

تิ

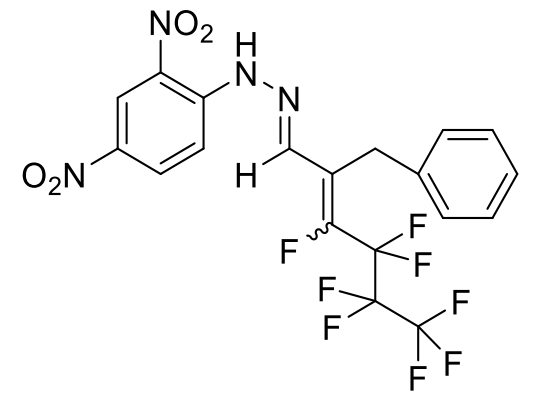

4b

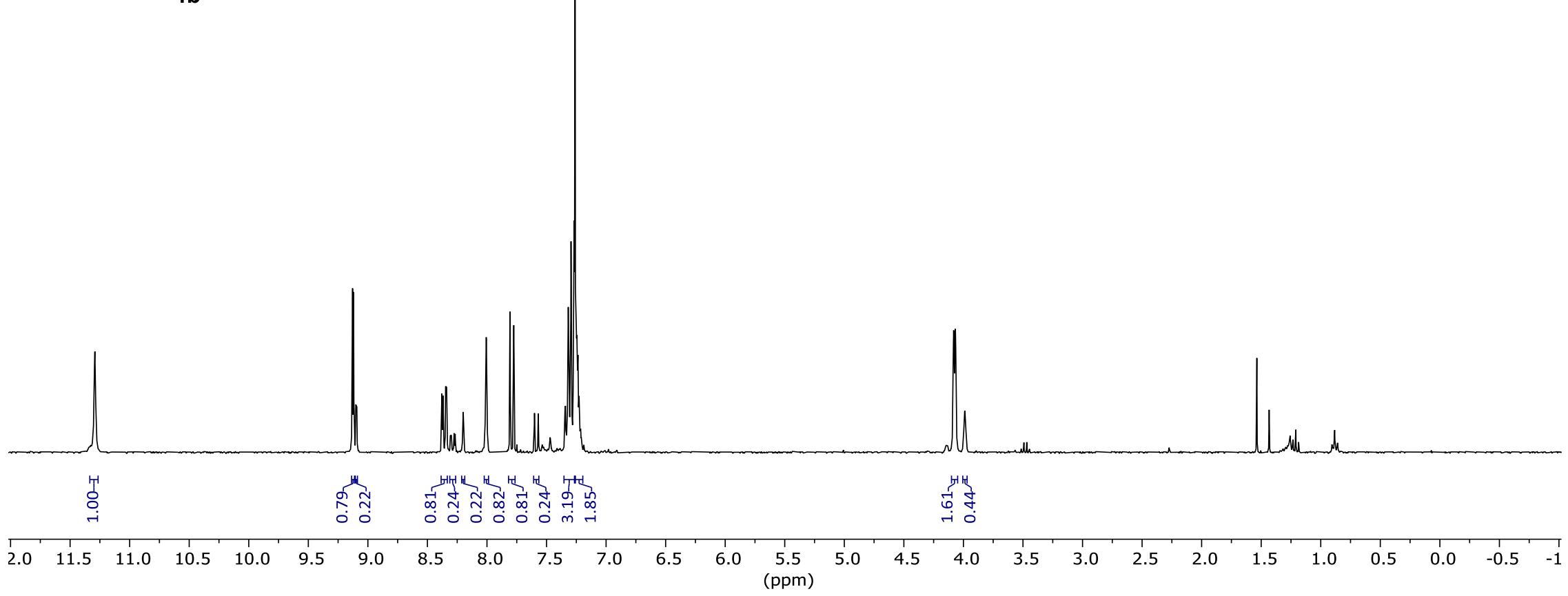



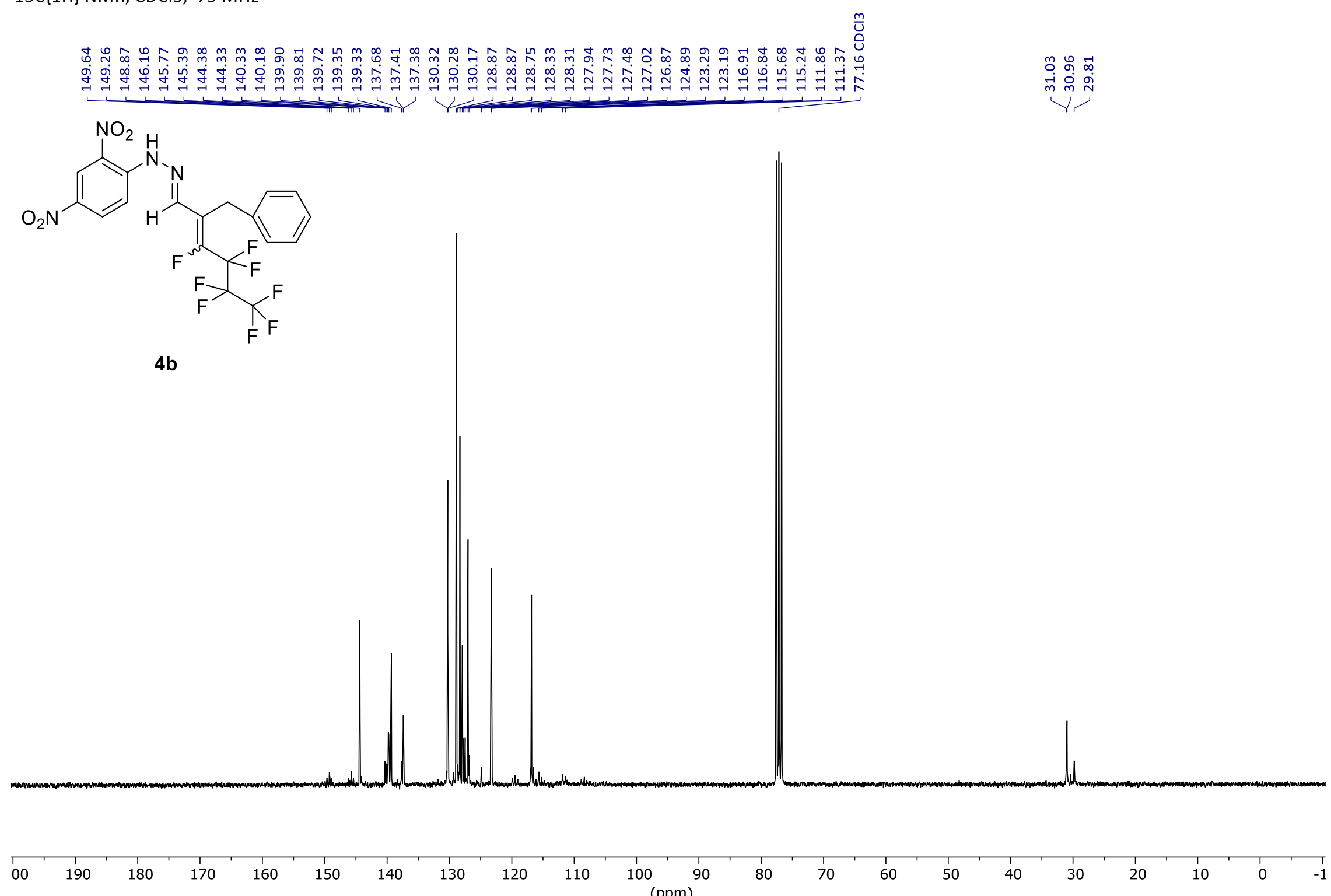
19F NMR, $\mathrm{CDCl} 3,282 \mathrm{MHz}$

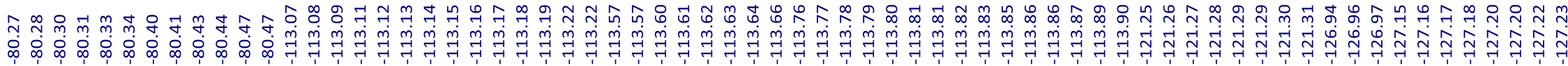

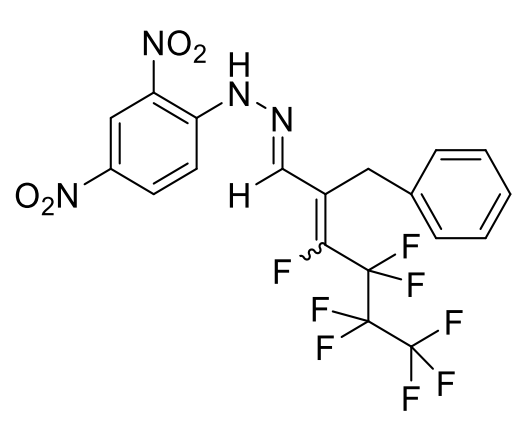

4b

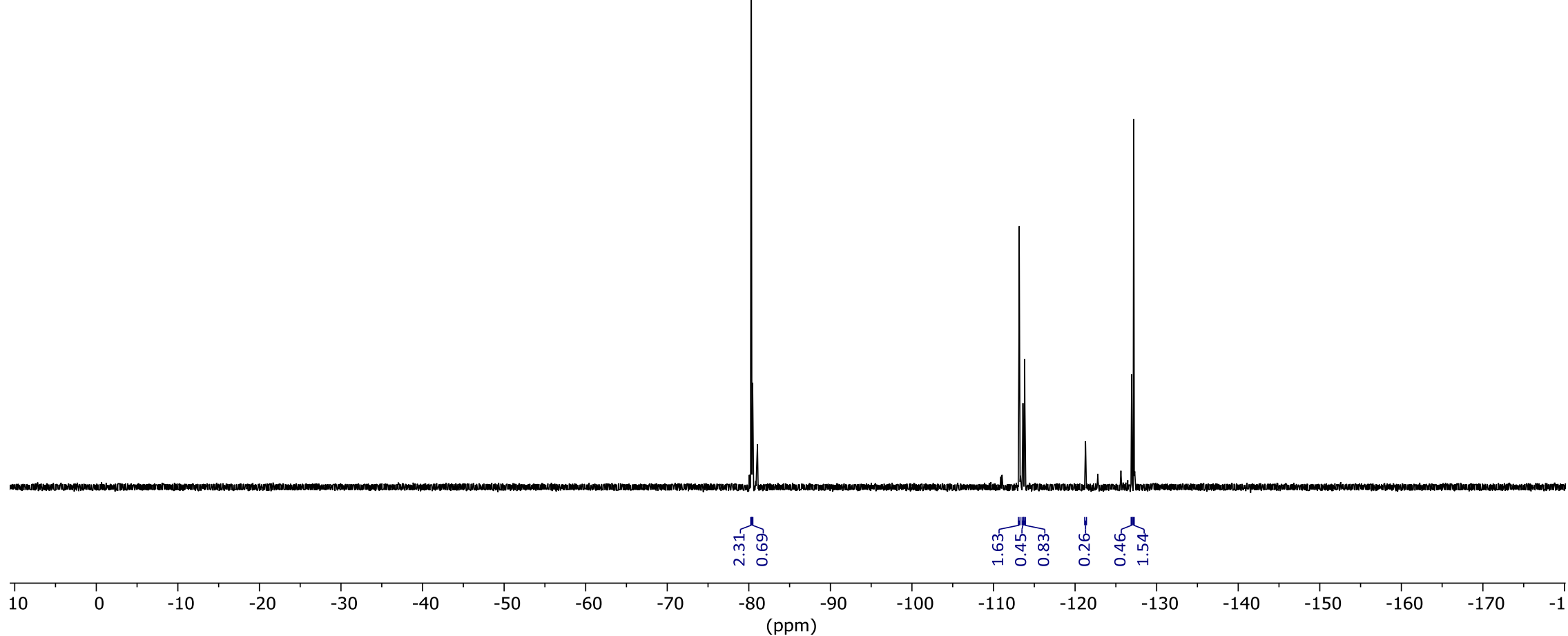


1H NMR, CDCl3, $600 \mathrm{MHz}$

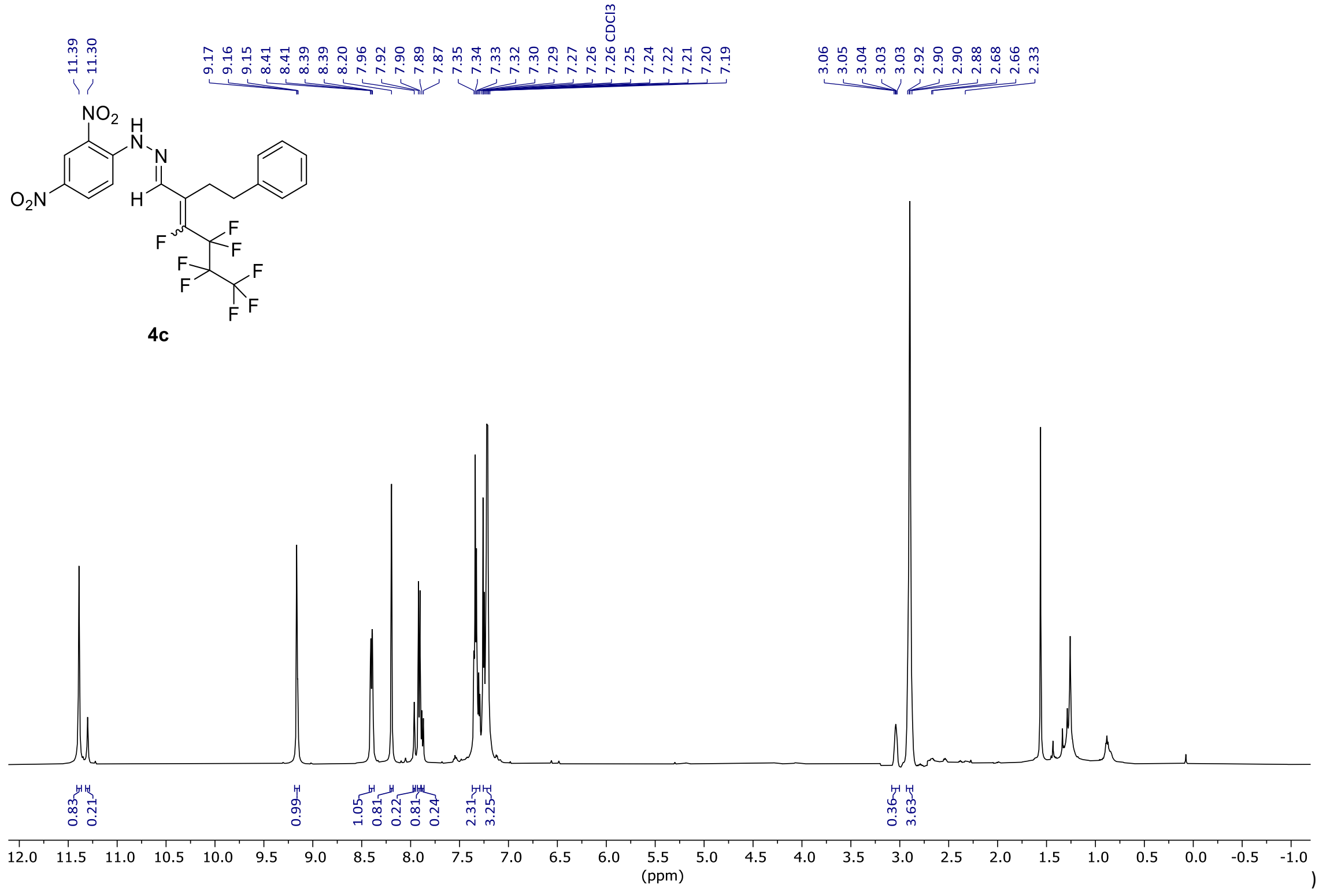


$13 \mathrm{C}\{1 \mathrm{H}\} \mathrm{NMR}, \mathrm{CDCl} 3,151 \mathrm{MHz}$

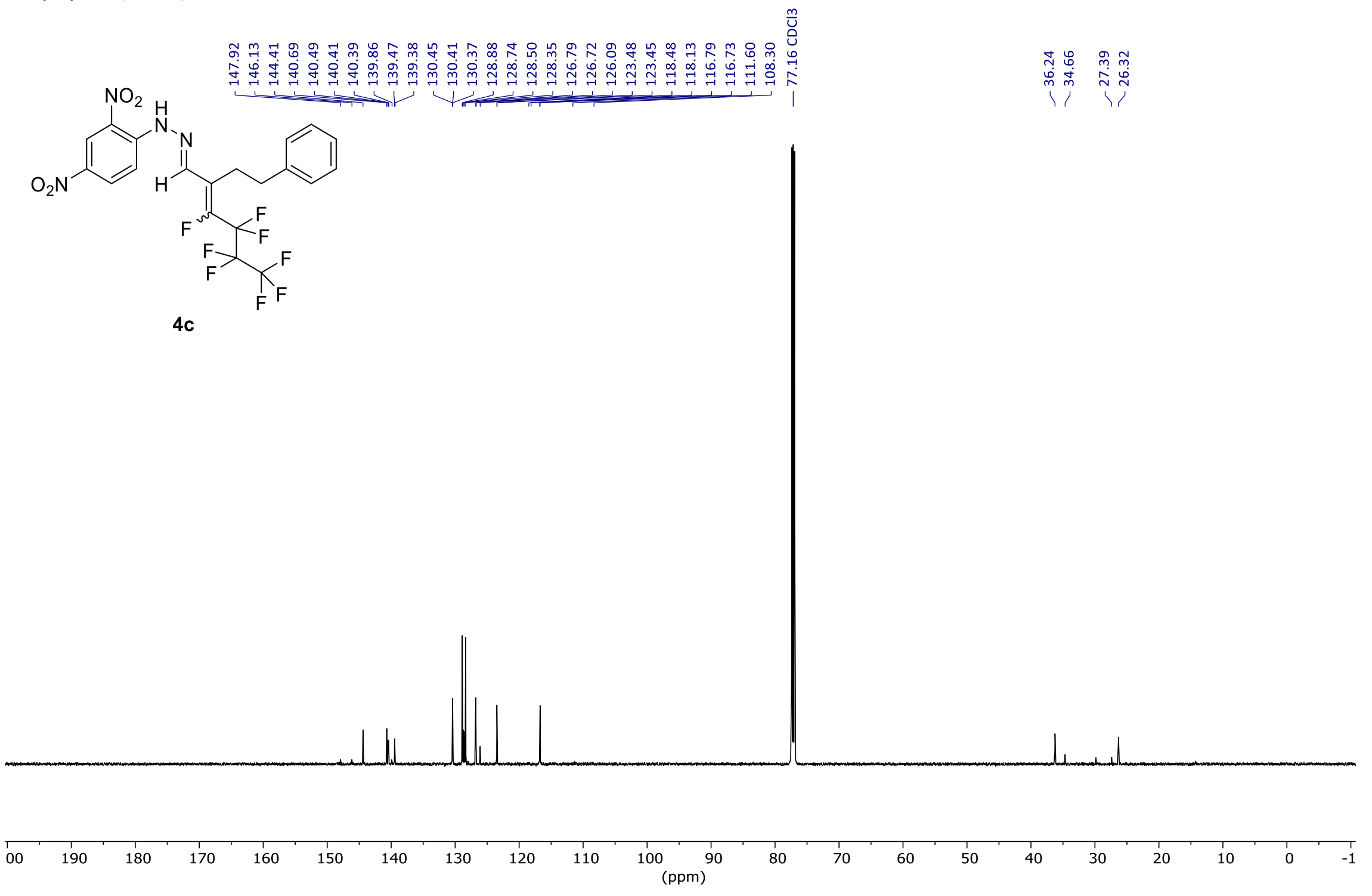


19F NMR, CDCl3, $282 \mathrm{MHz}$

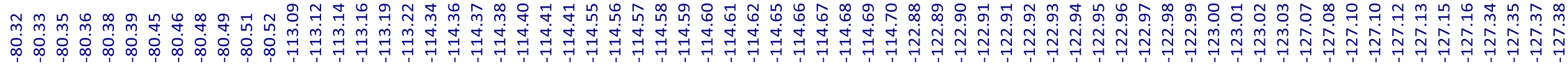

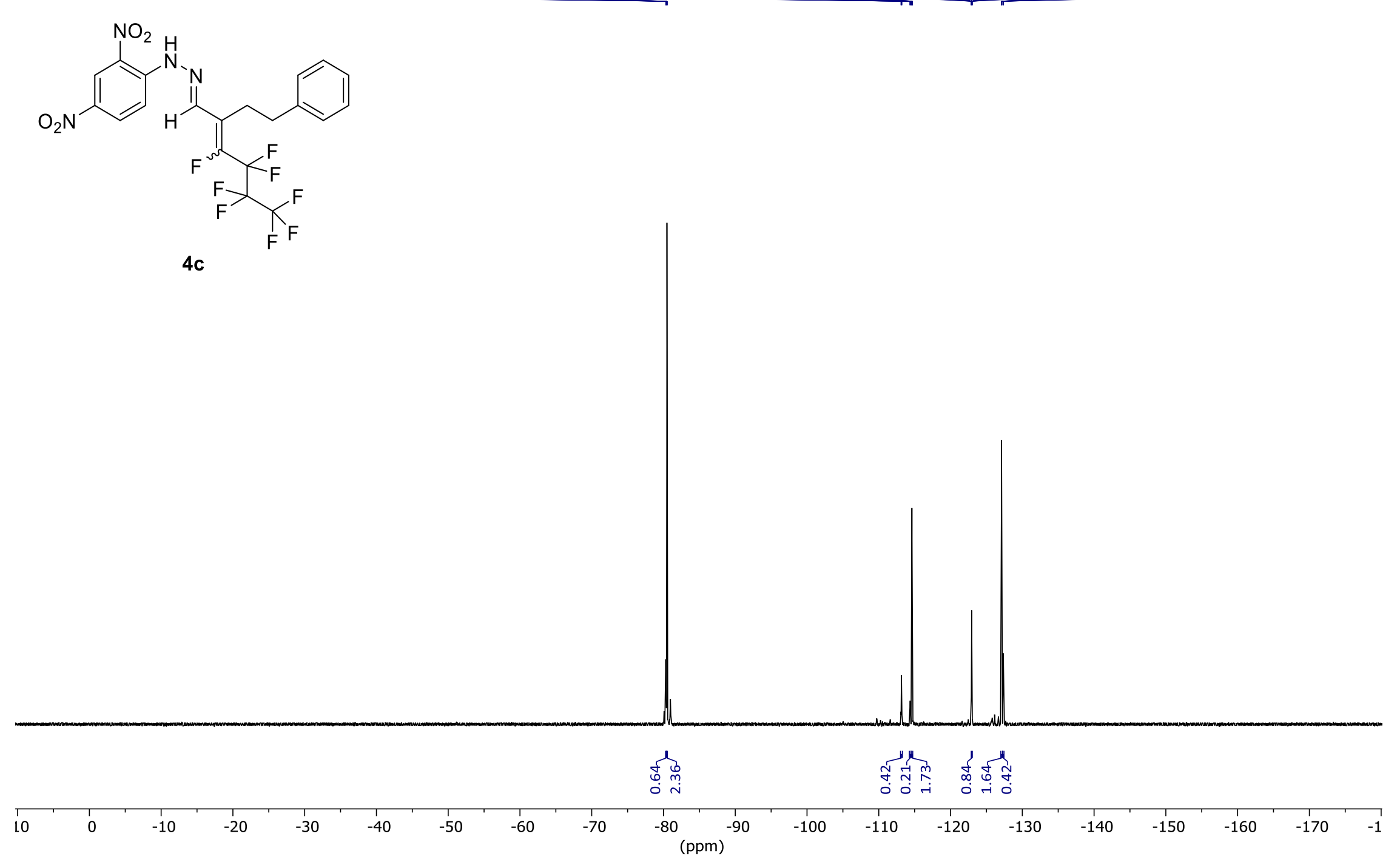


1H NMR, $\mathrm{CDCl} 3,300 \mathrm{MHz}$

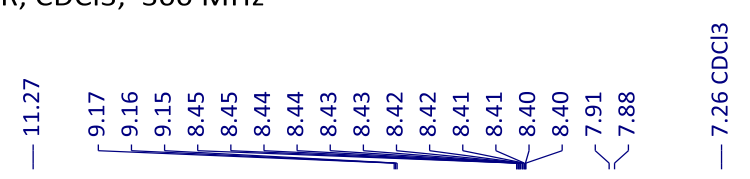

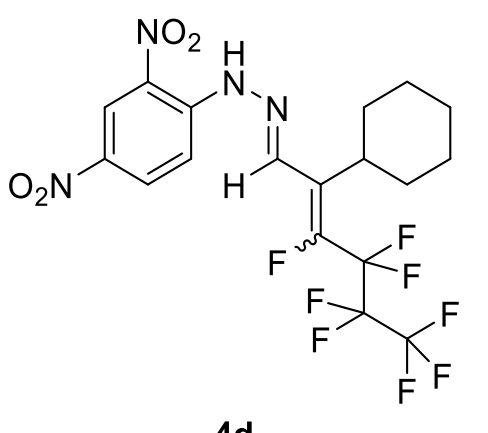

울 궁옥

$4 d$

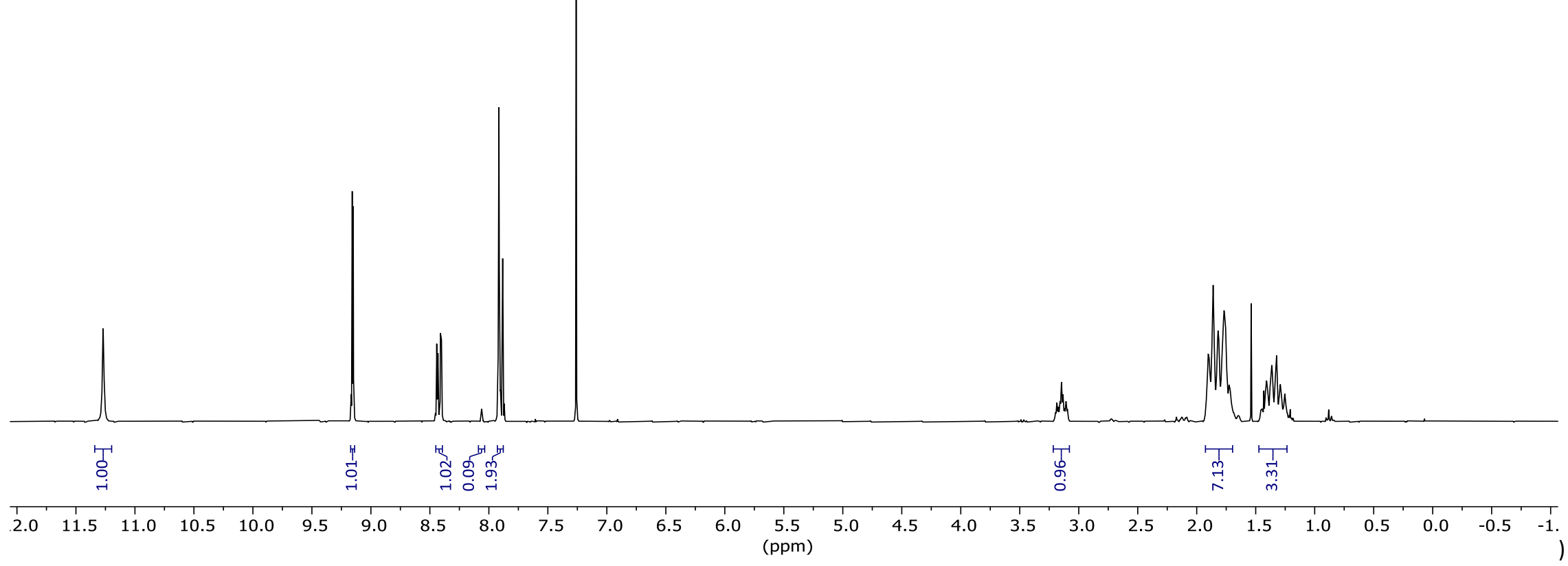




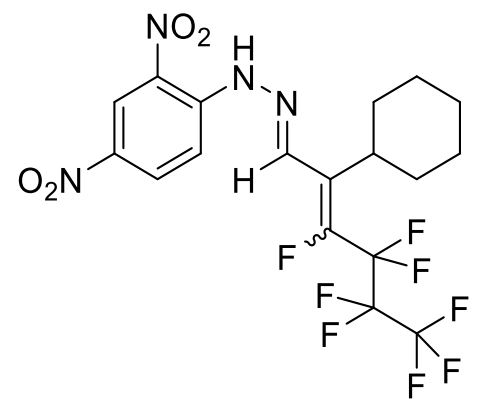

$4 d$

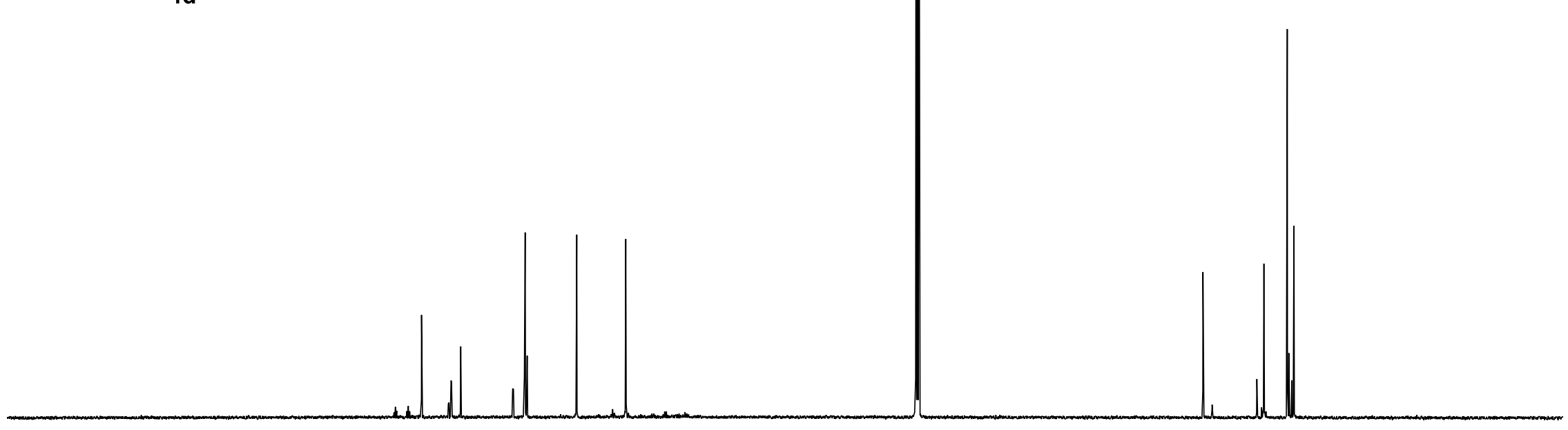

$\begin{array}{lll}180 & 170 \quad 160\end{array}$

$150 \quad 140$

$130 \quad 120$

$110 \quad 100$

(ppm) 
19F NMR, CDCl3, $565 \mathrm{MHz}$

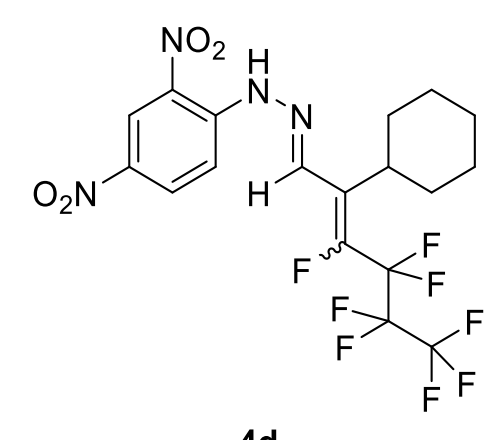

$4 d$

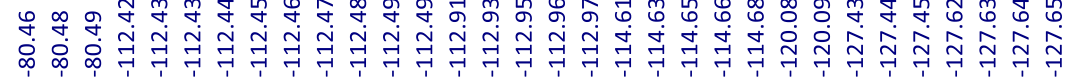

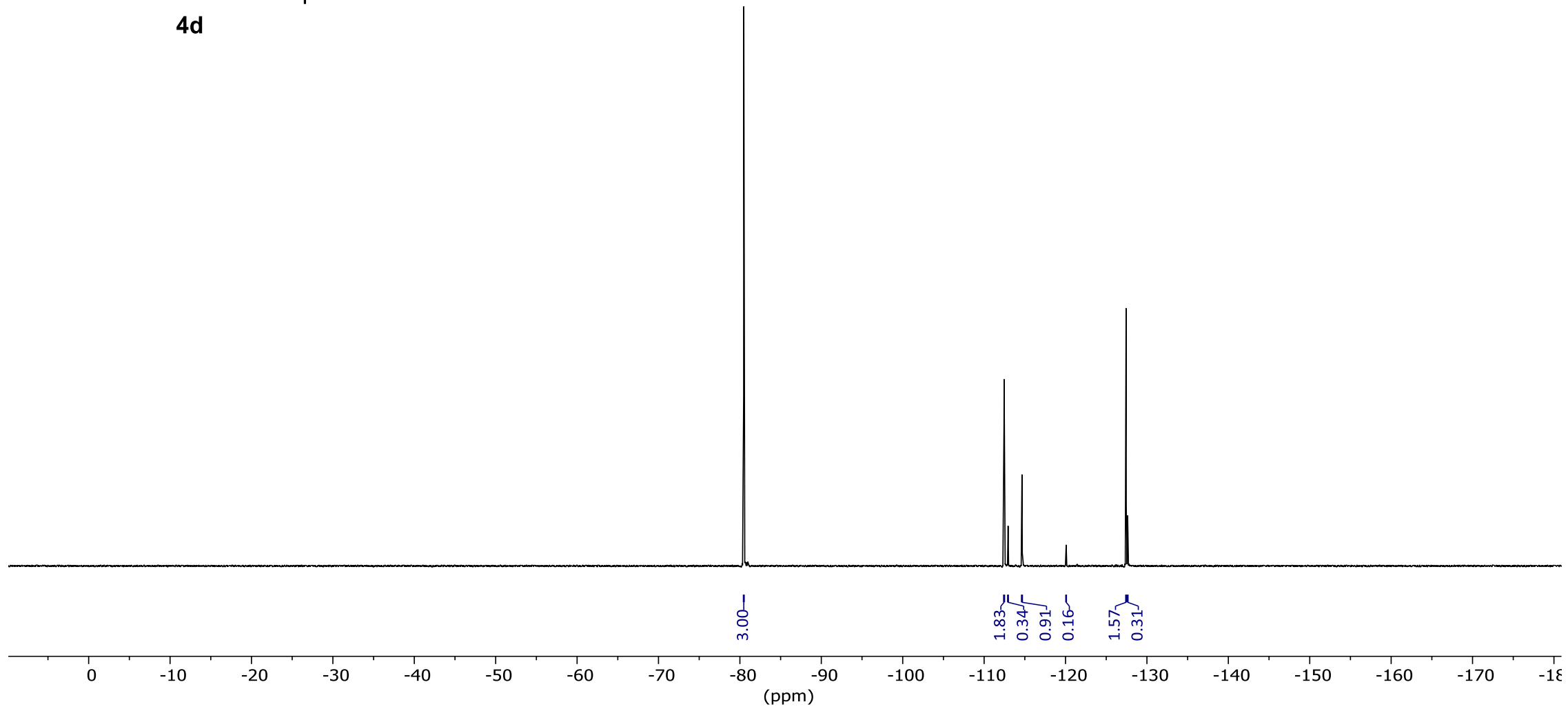




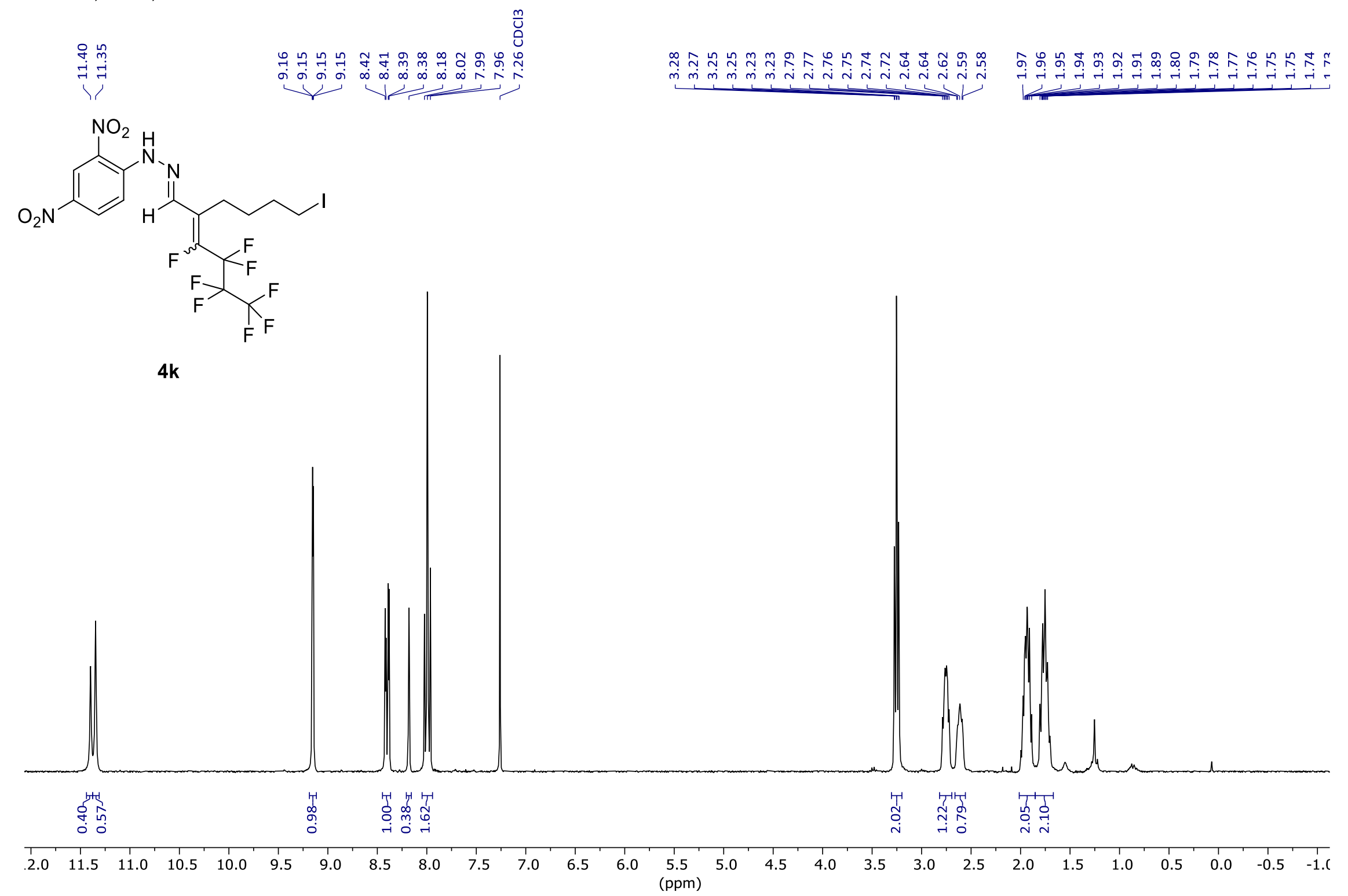




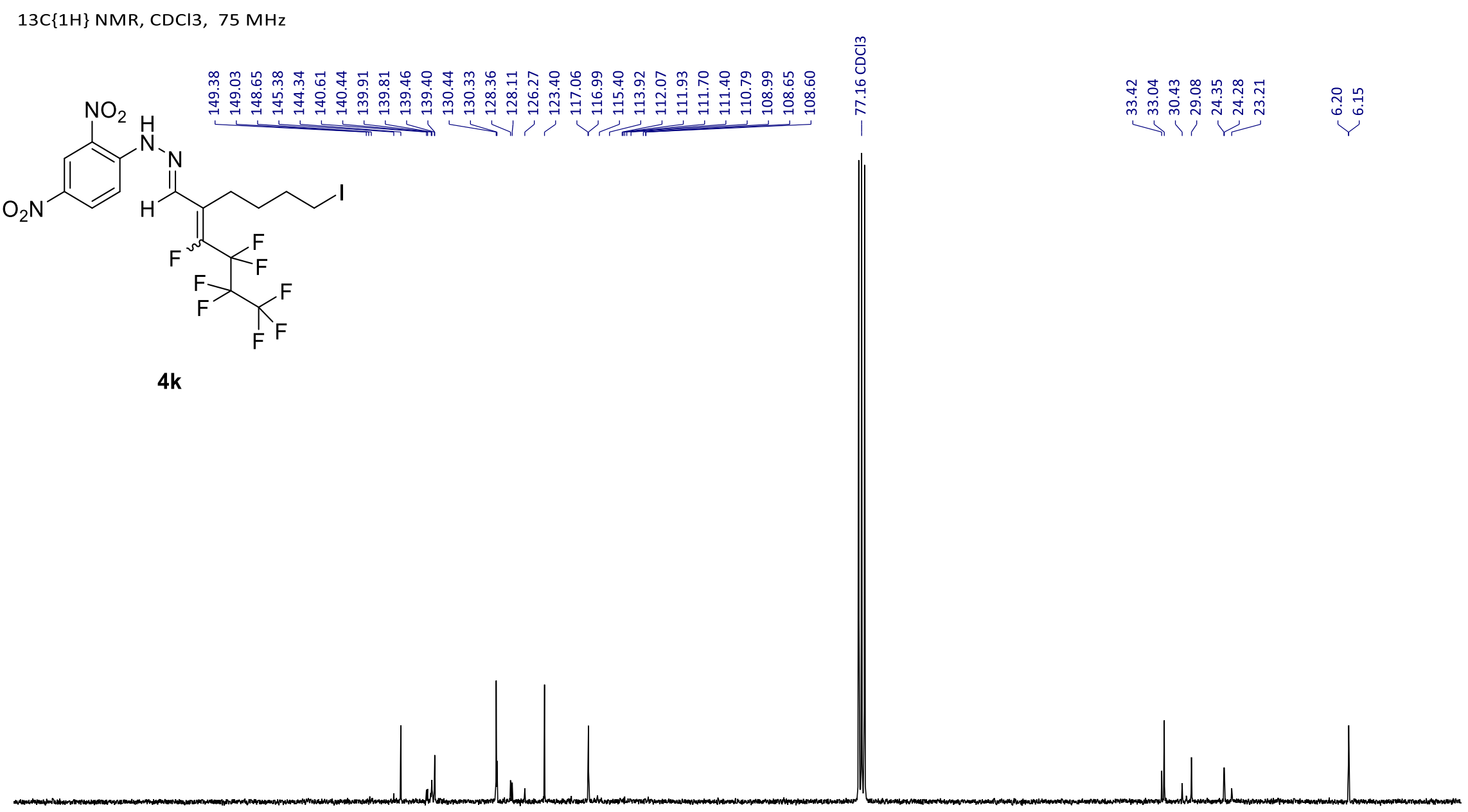

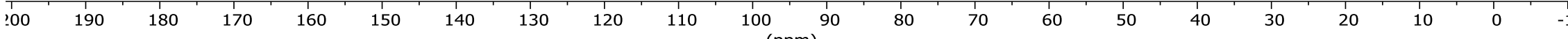


19F NMR, CDCl3, $282 \mathrm{MHz}$

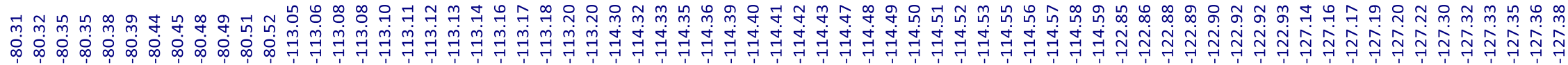

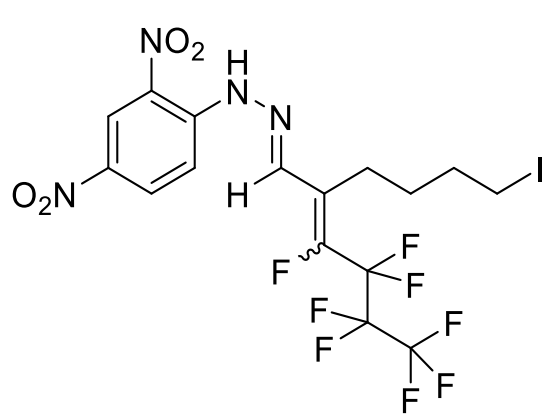

4k

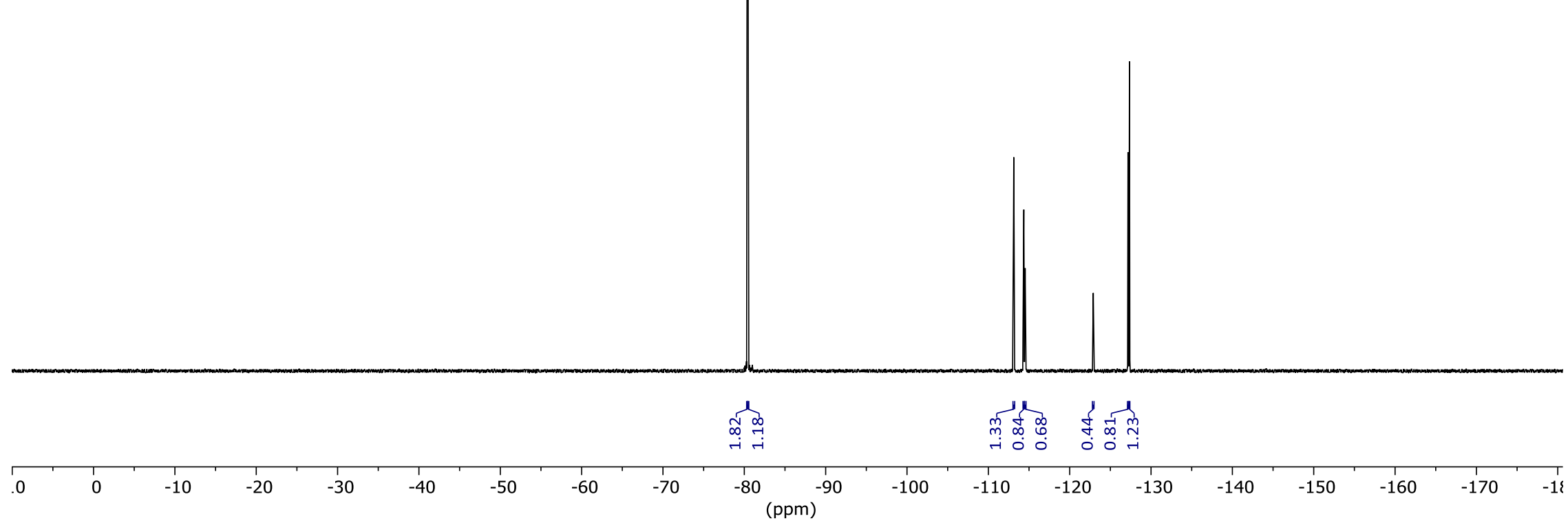




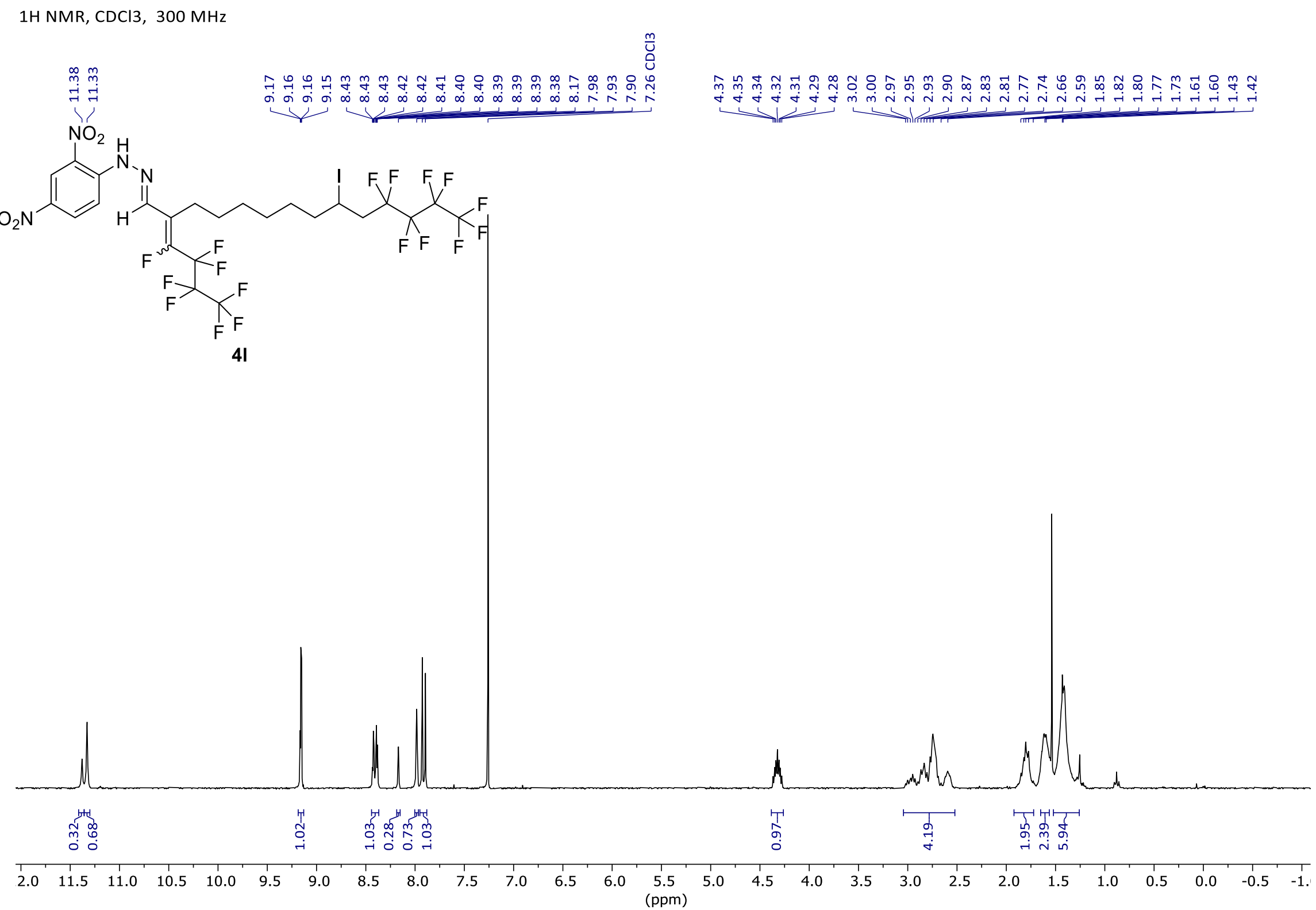



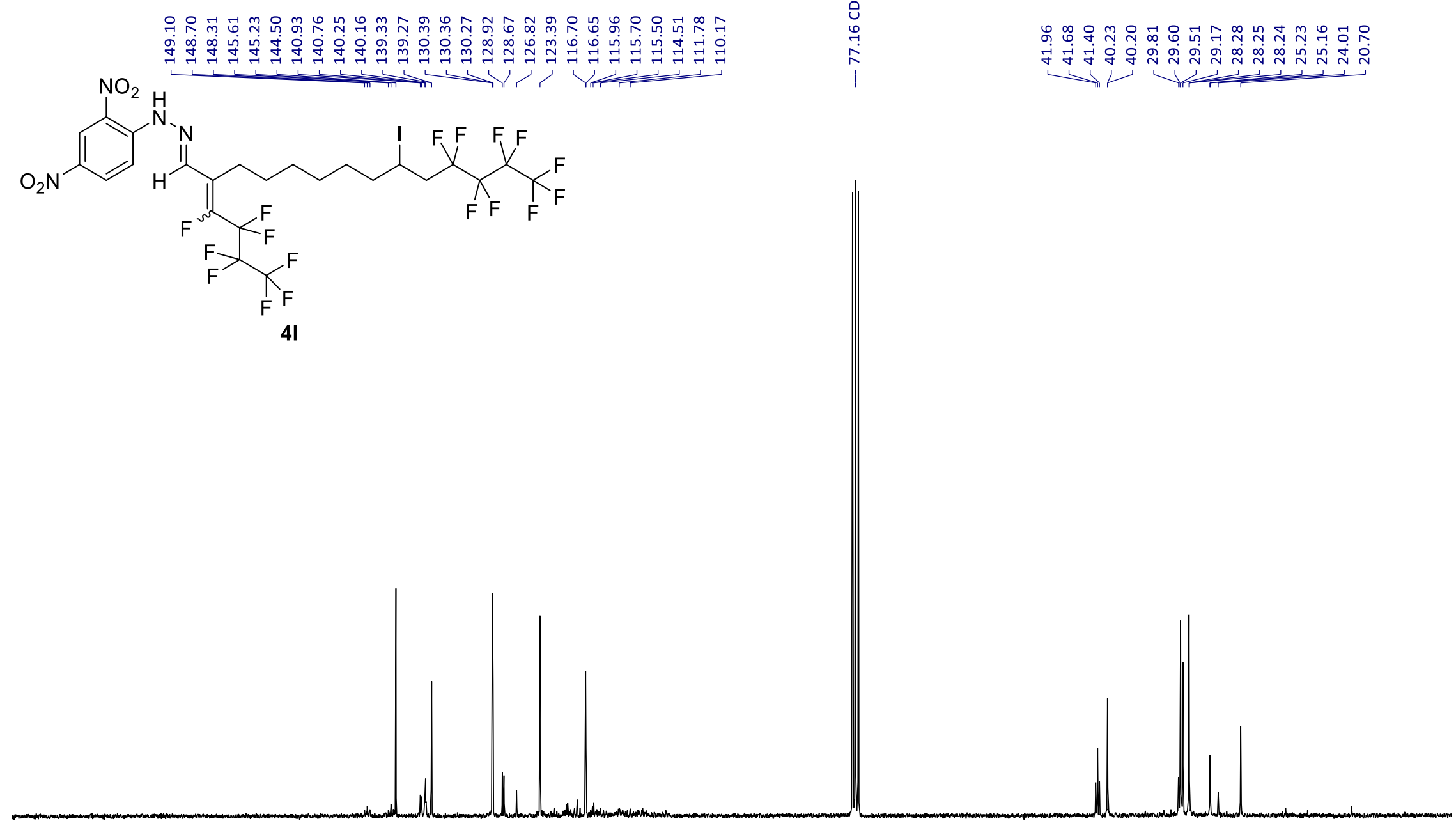

$\stackrel{1}{100}$

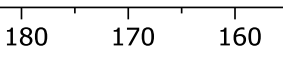


19F NMR, CDCl3, $282 \mathrm{MHz}$

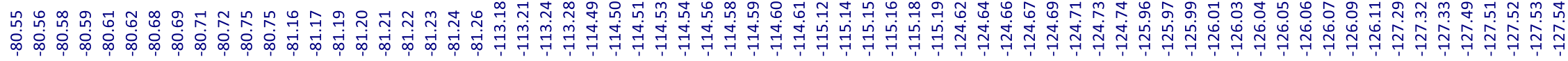
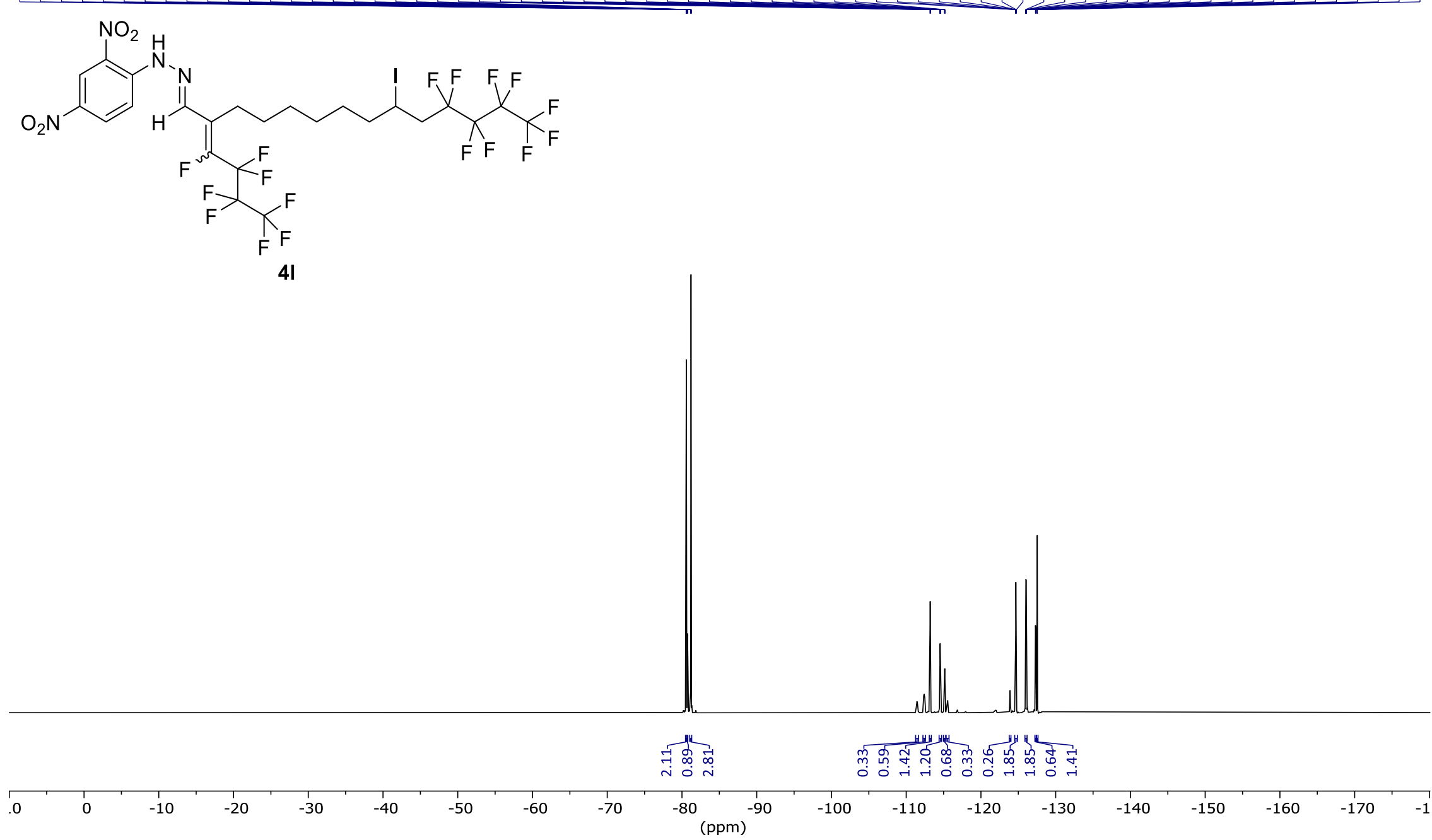


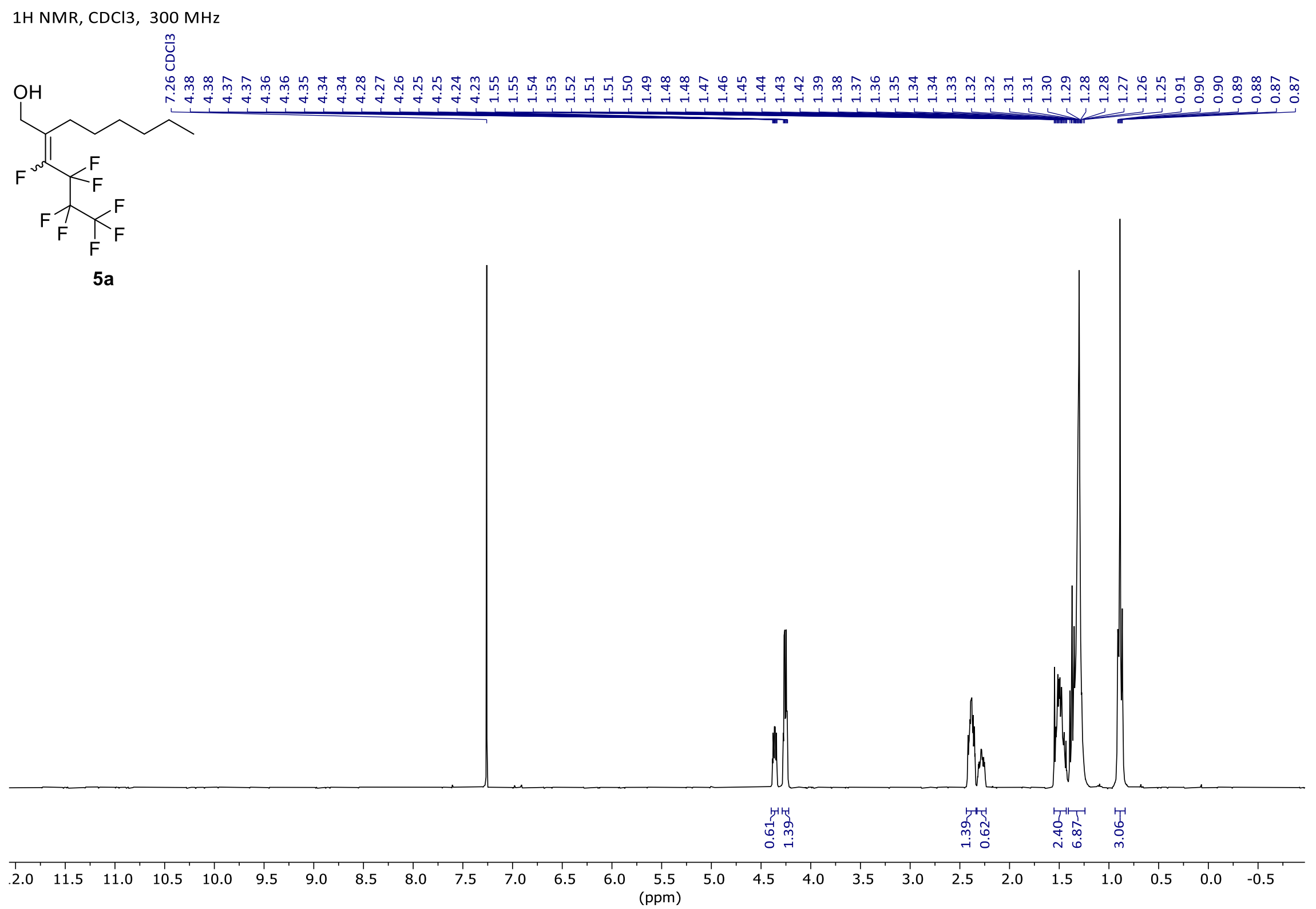




\section{$13 \mathrm{C}\{1 \mathrm{H}\} \mathrm{NMR}, \mathrm{CDCl} 3,75 \mathrm{MHz}$}

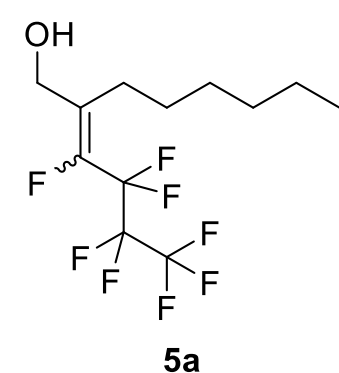

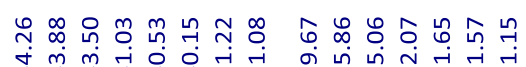

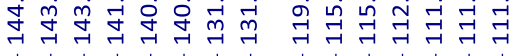

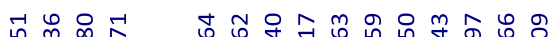

舟然的

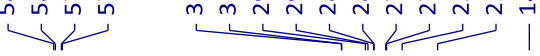

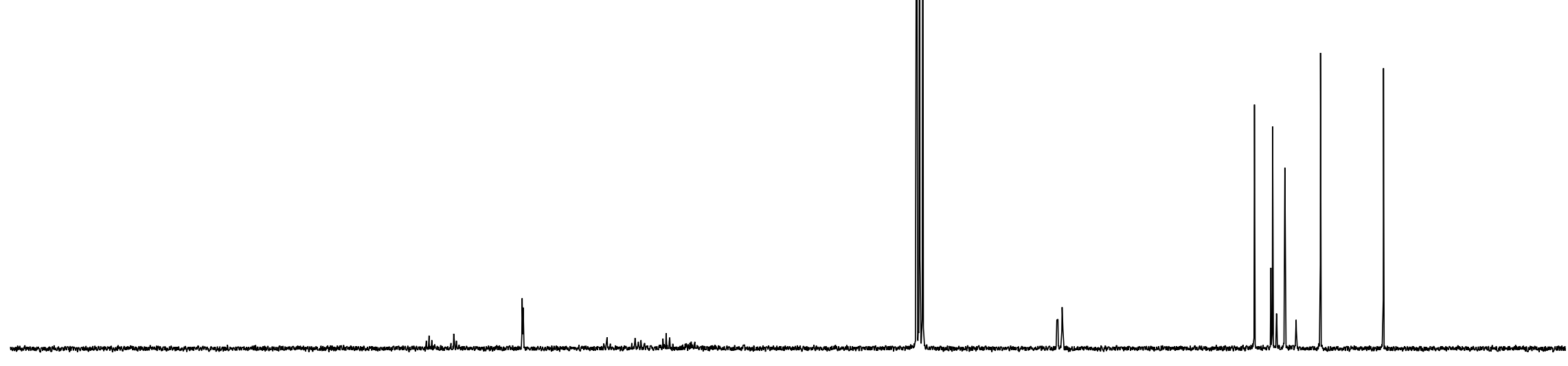


19F NMR, CDCl3, $282 \mathrm{MHz}$

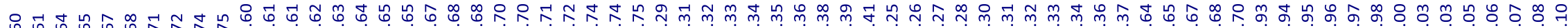

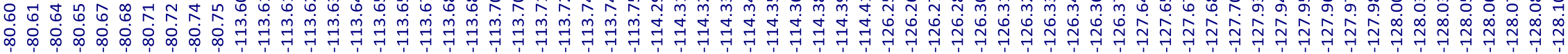
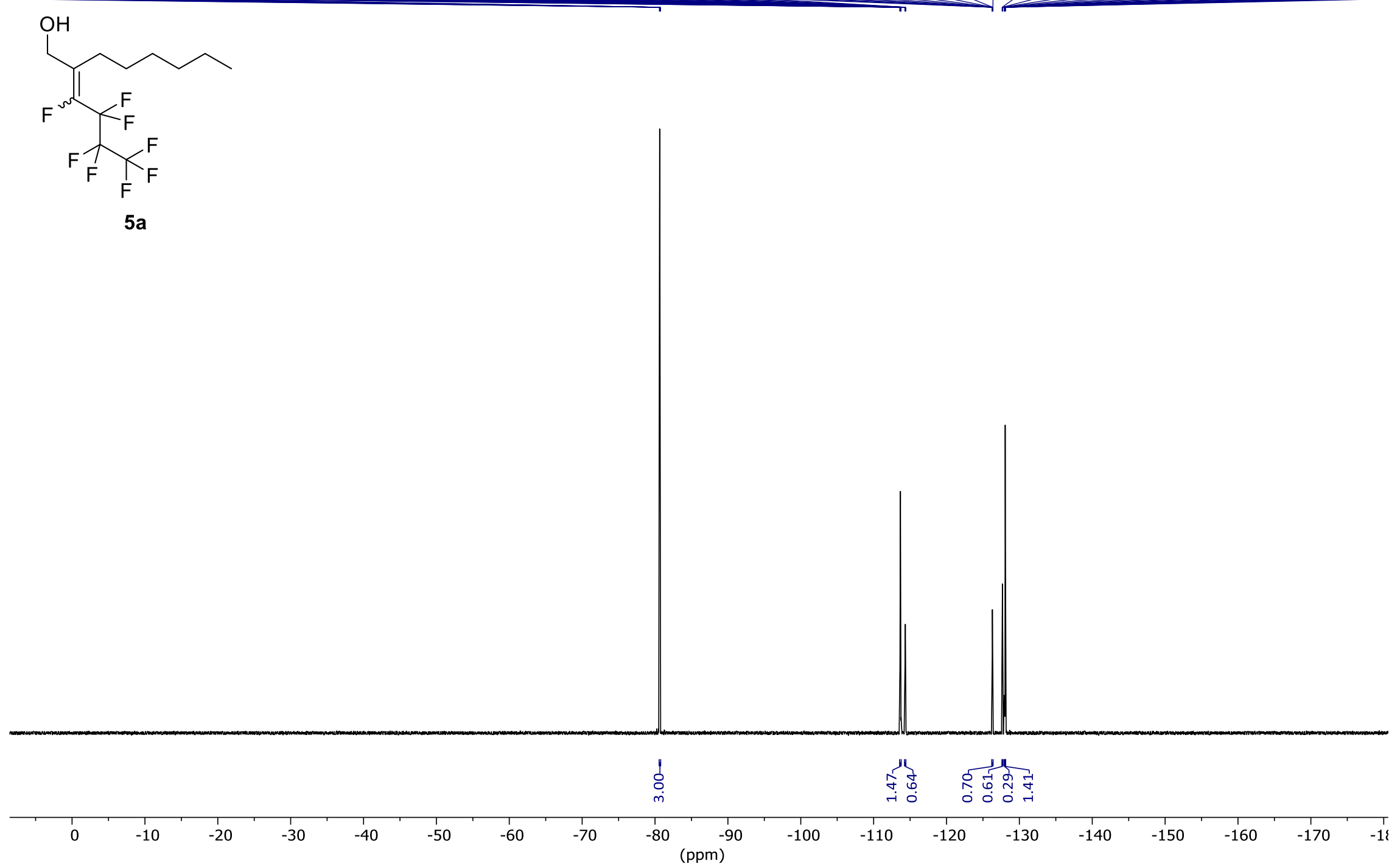


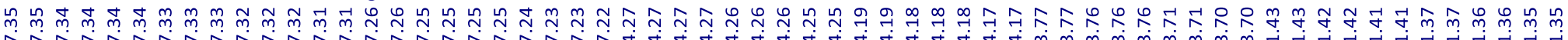

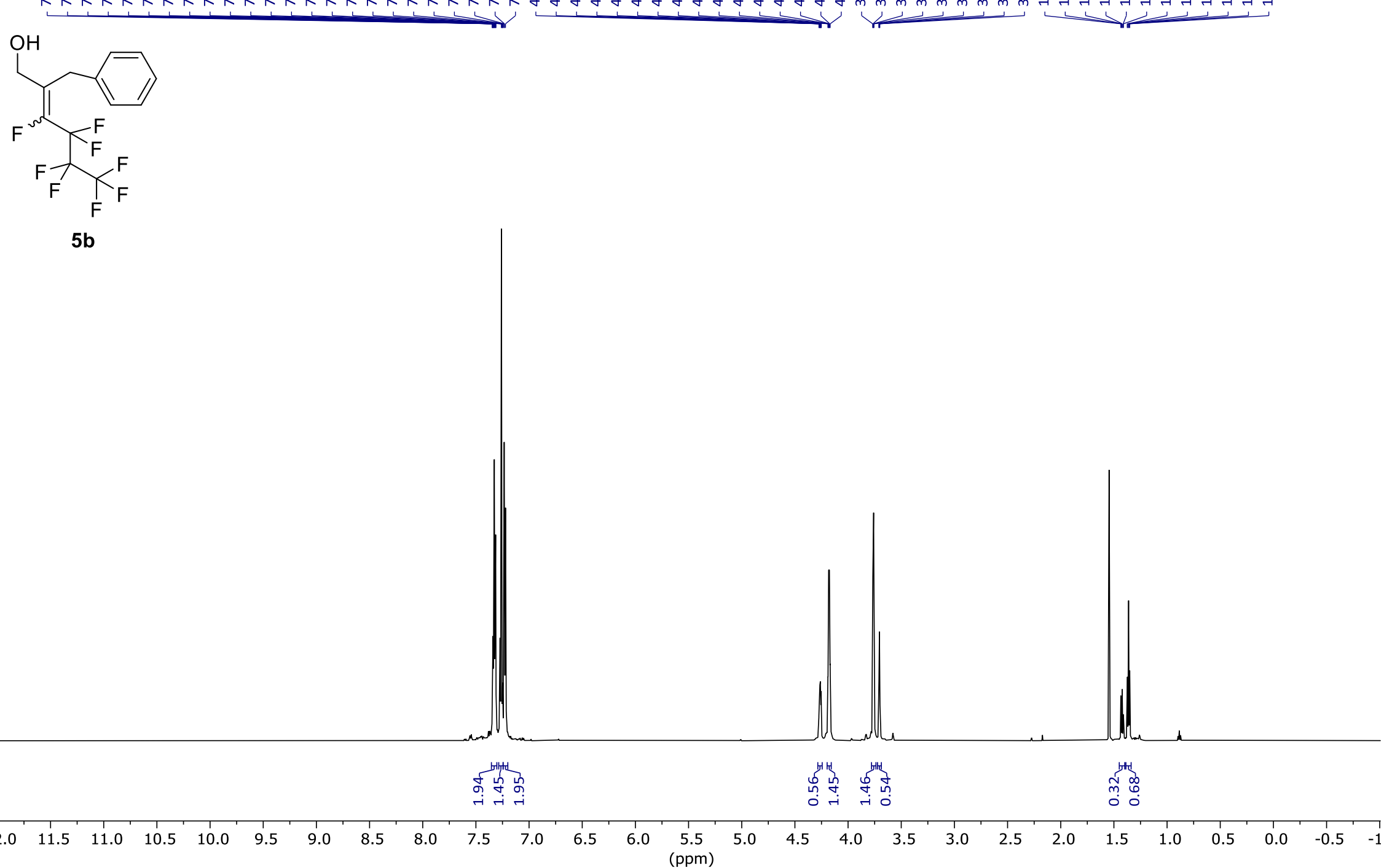




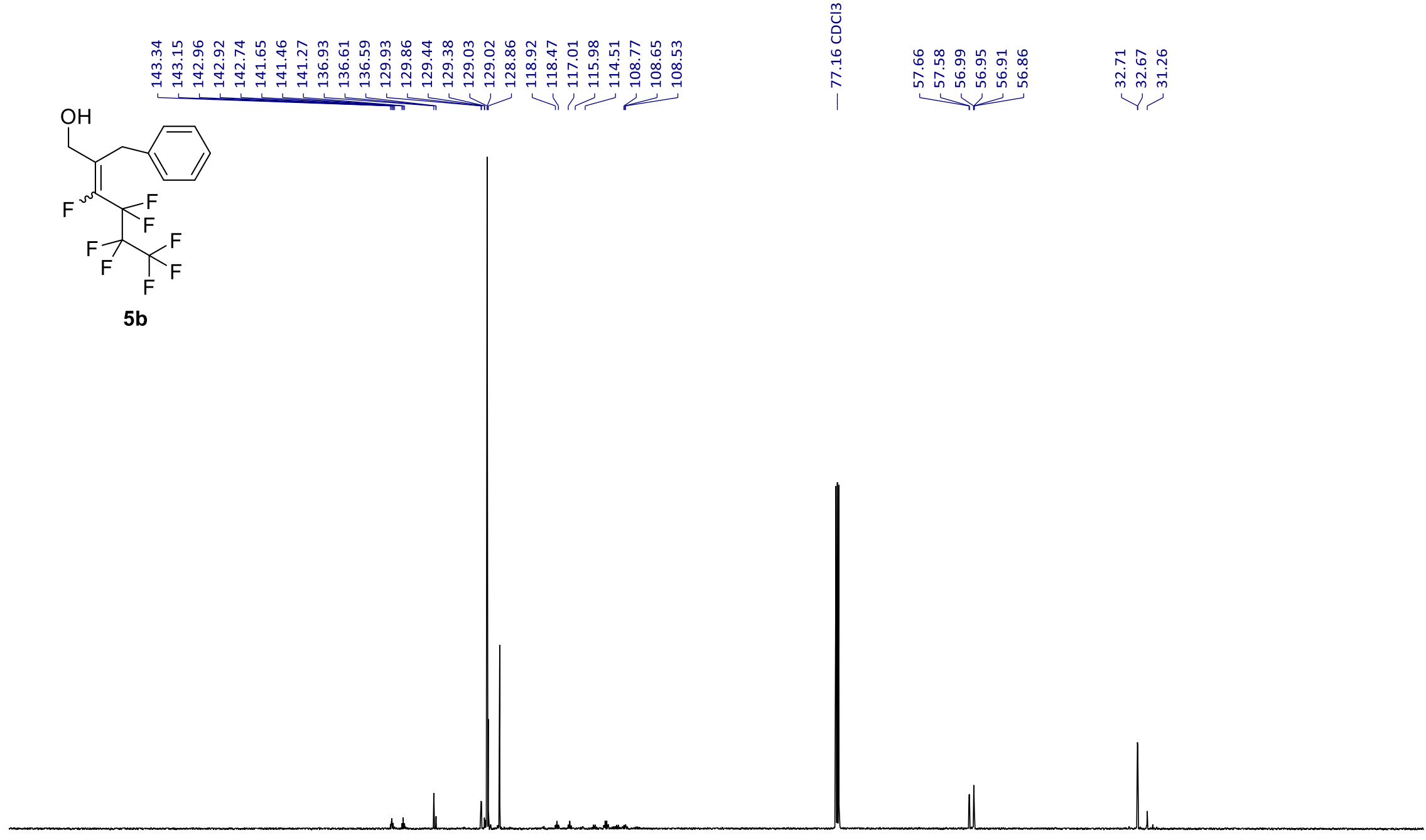

$190 \quad 180$ 
19F NMR, CDCl3, $565 \mathrm{MHz}$

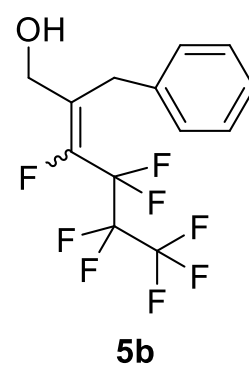

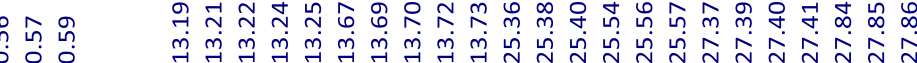

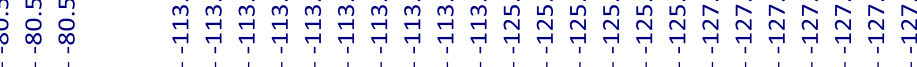

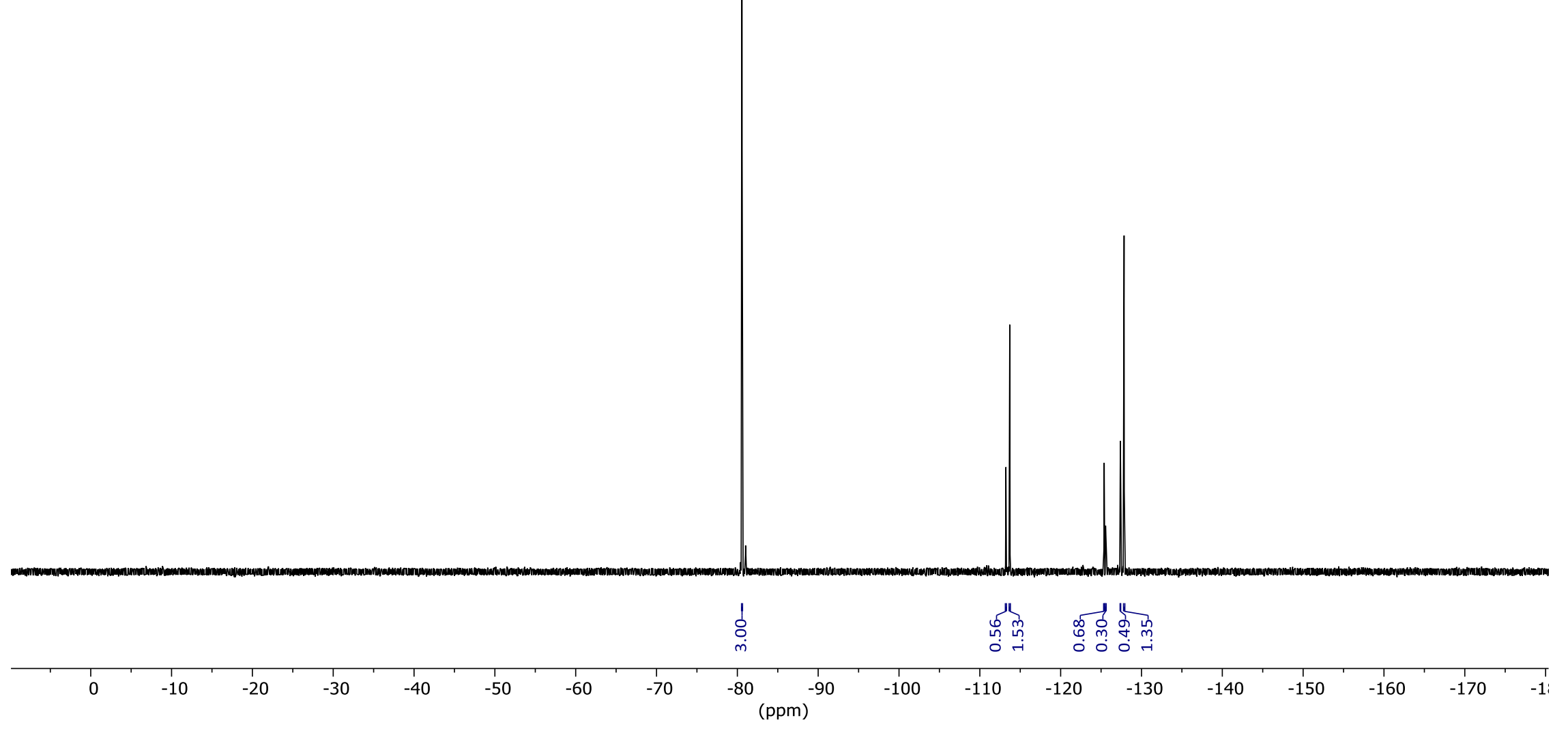




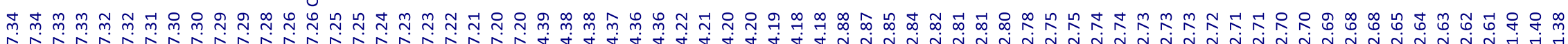

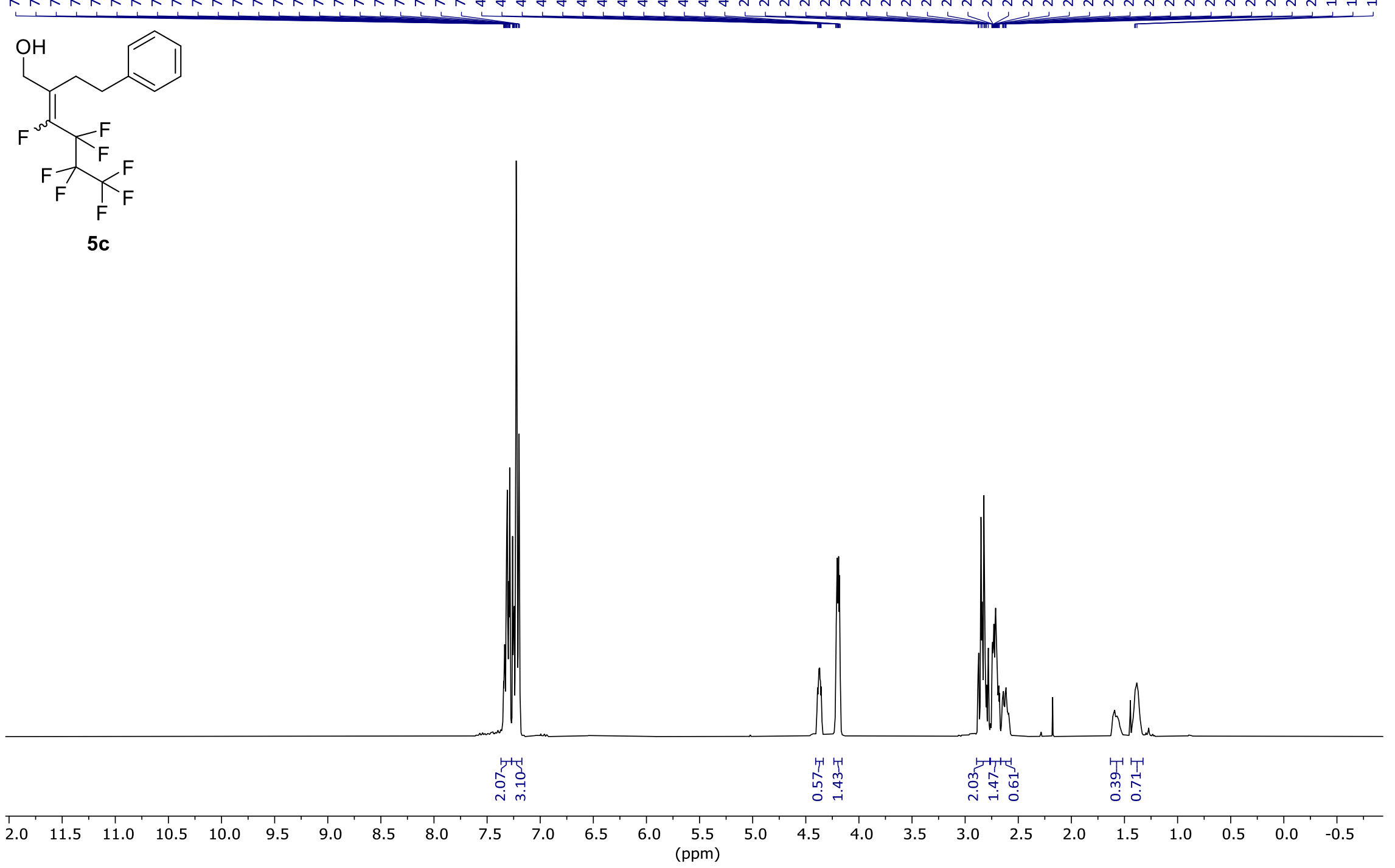


$13 \mathrm{C}\{1 \mathrm{H}\} \mathrm{NMR}, \mathrm{CDCl} 3,75 \mathrm{MHz}$
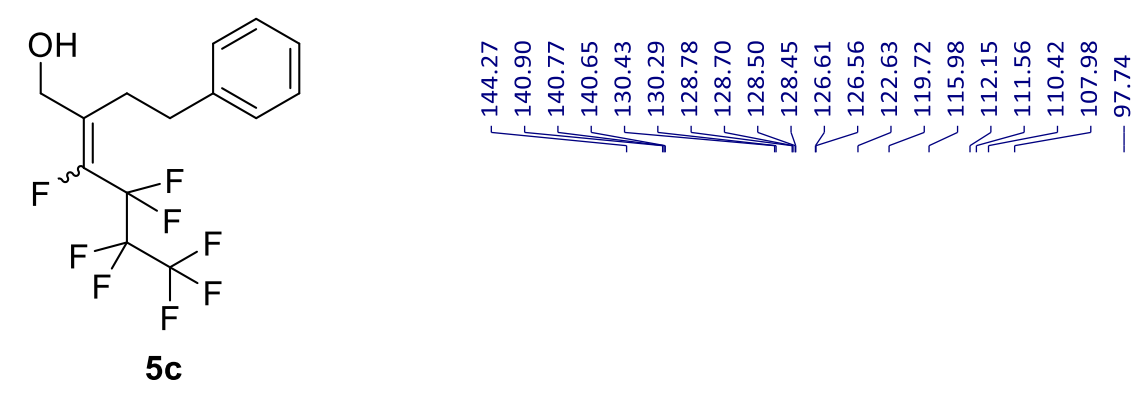

$\underline{m}$

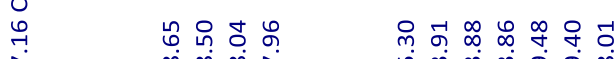

i $\quad$ i

$1+$

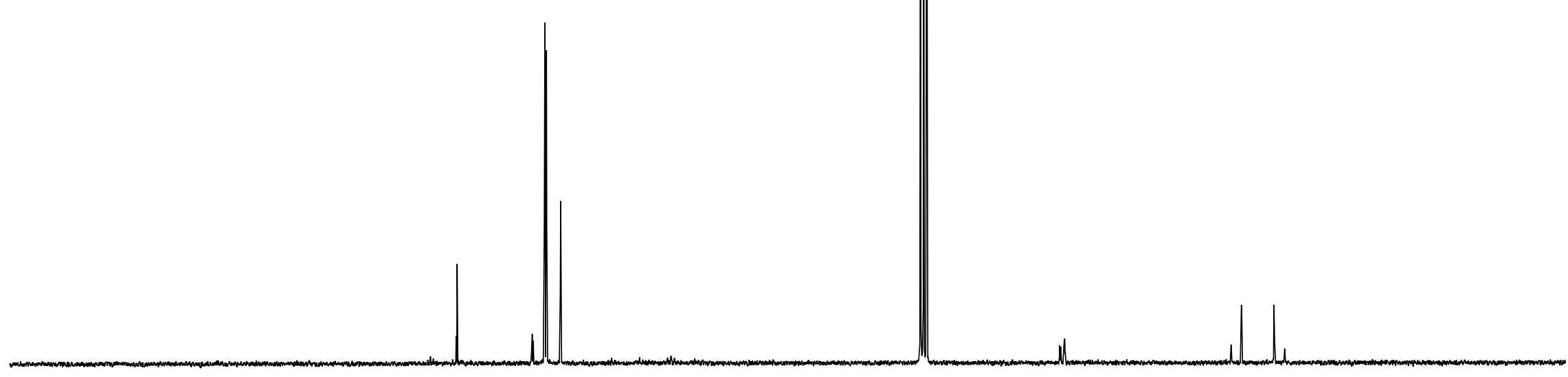

200 
19F NMR, CDCl3, $282 \mathrm{MHz}$

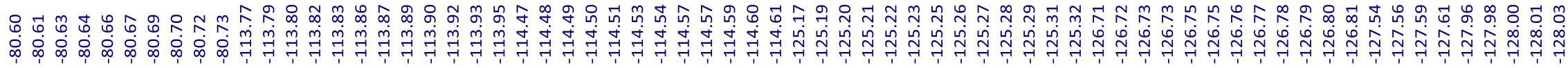
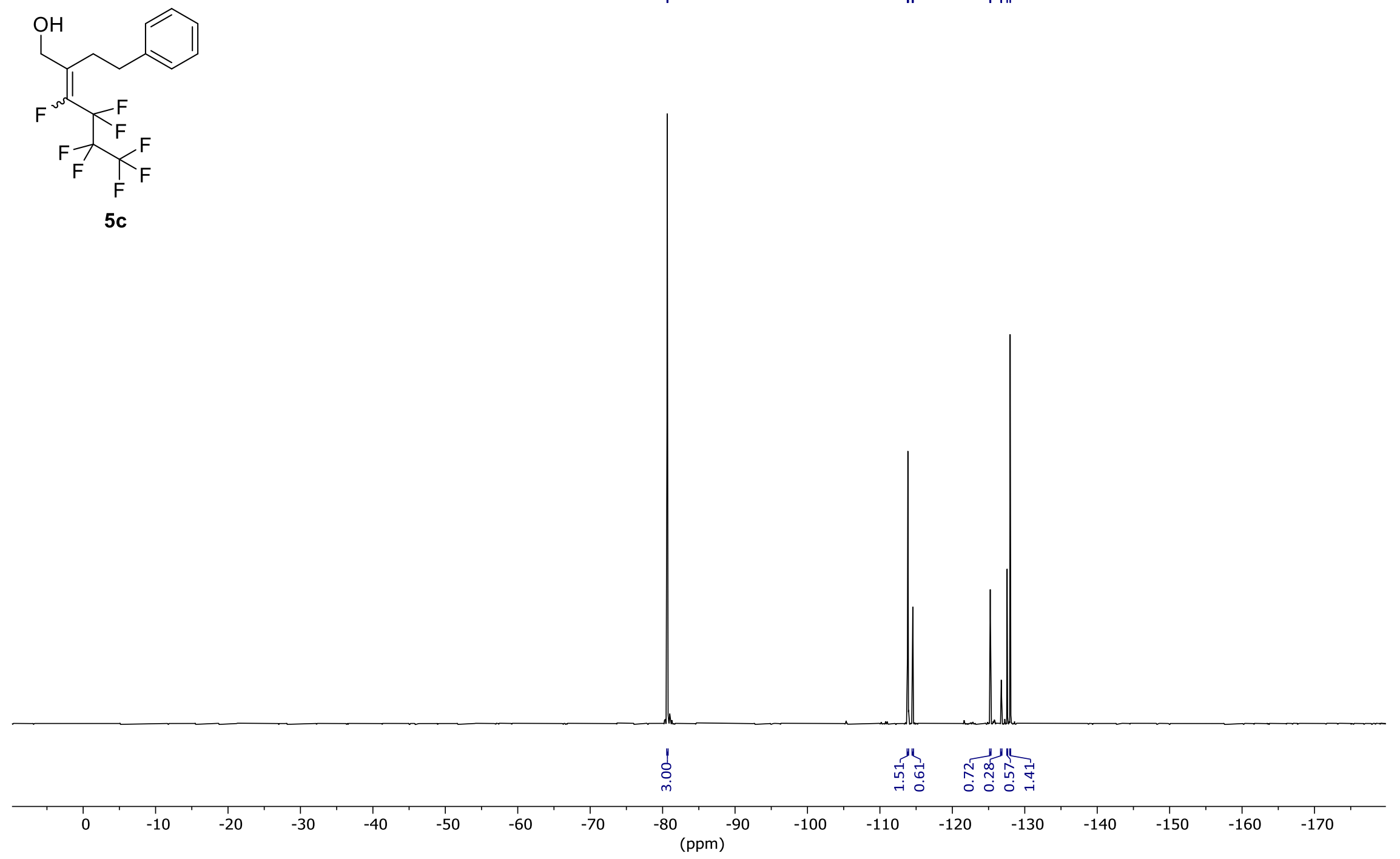
$1 \mathrm{H} \mathrm{NMR}, \mathrm{CDCl} 3,300 \mathrm{MHz}$

竞

定过 年
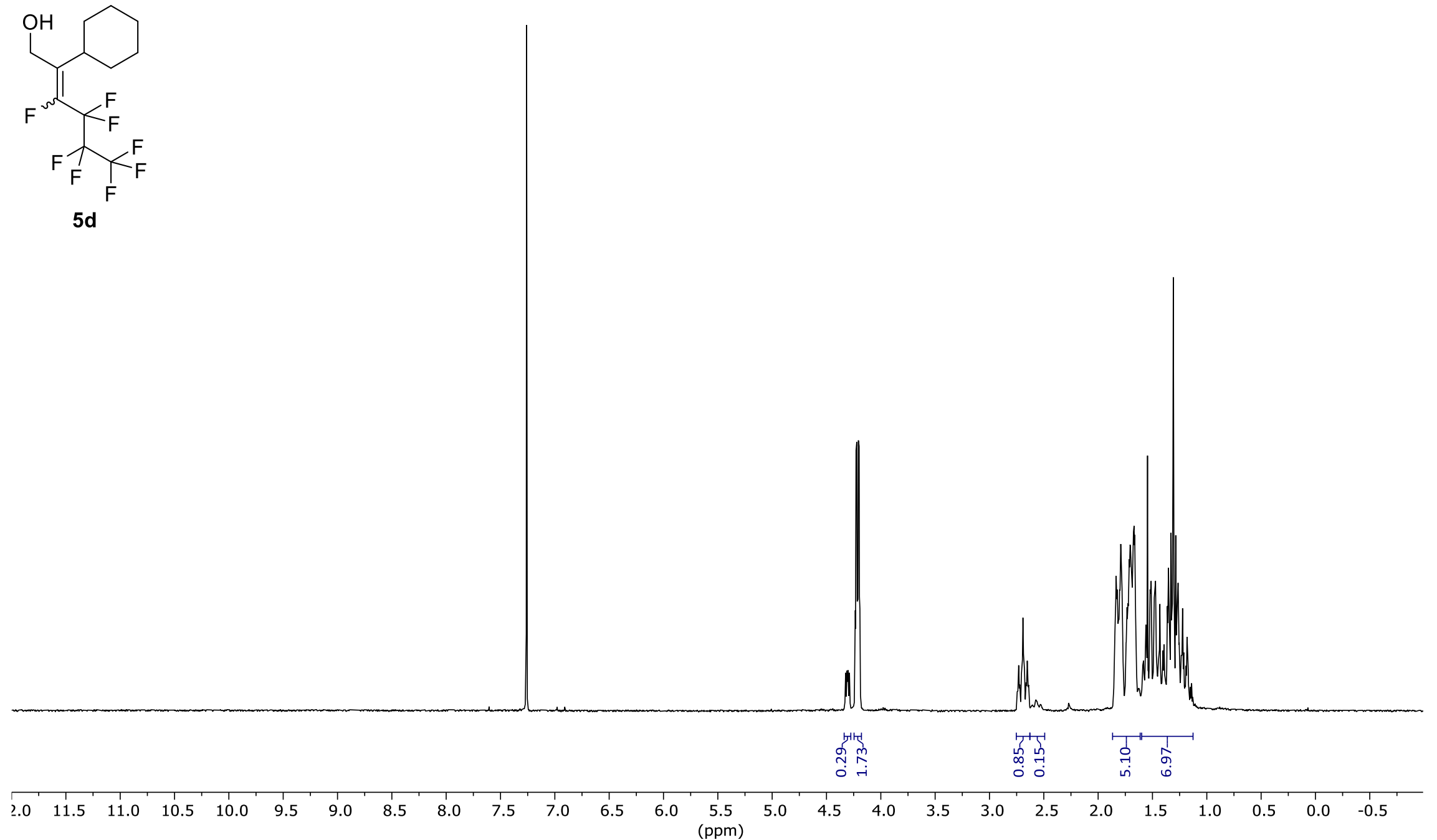
$13 \mathrm{C}\{1 \mathrm{H}\} \mathrm{NMR}, \mathrm{CDCl} 3,75 \mathrm{MHz}$

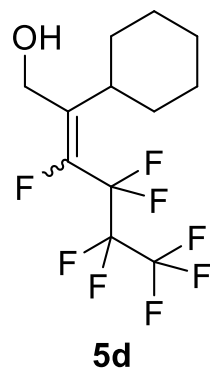

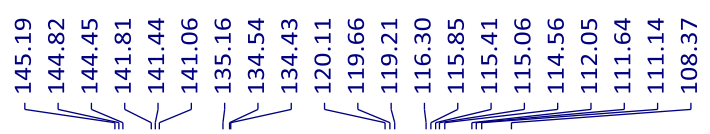

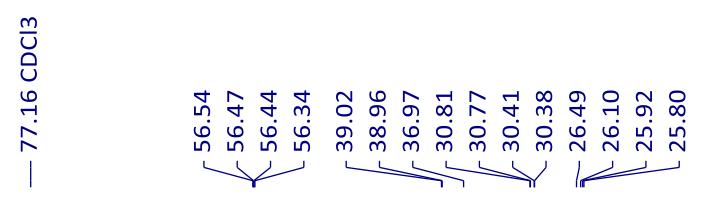

$5 d$

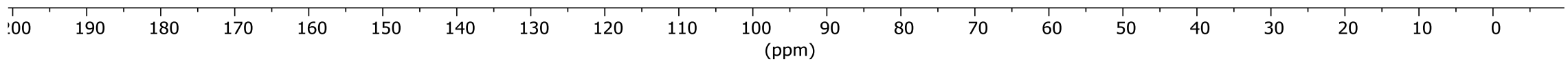

S82 
19F NMR, CDCl3, $282 \mathrm{MHz}$

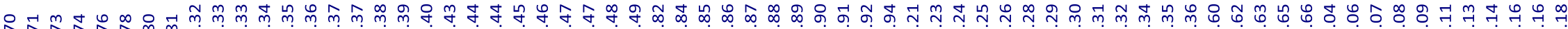

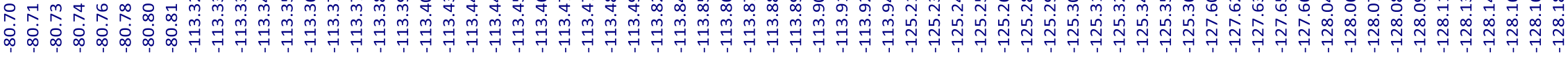
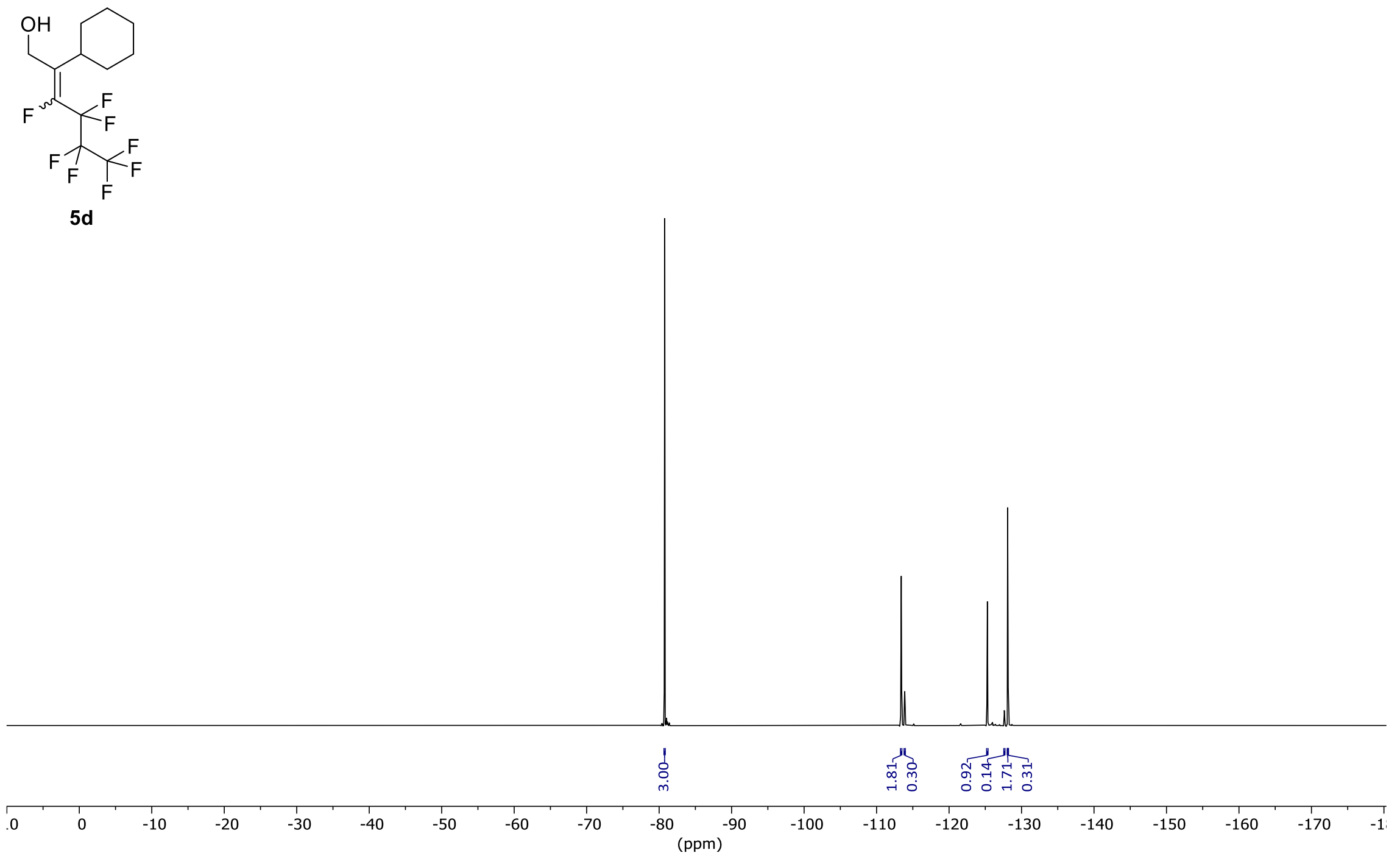


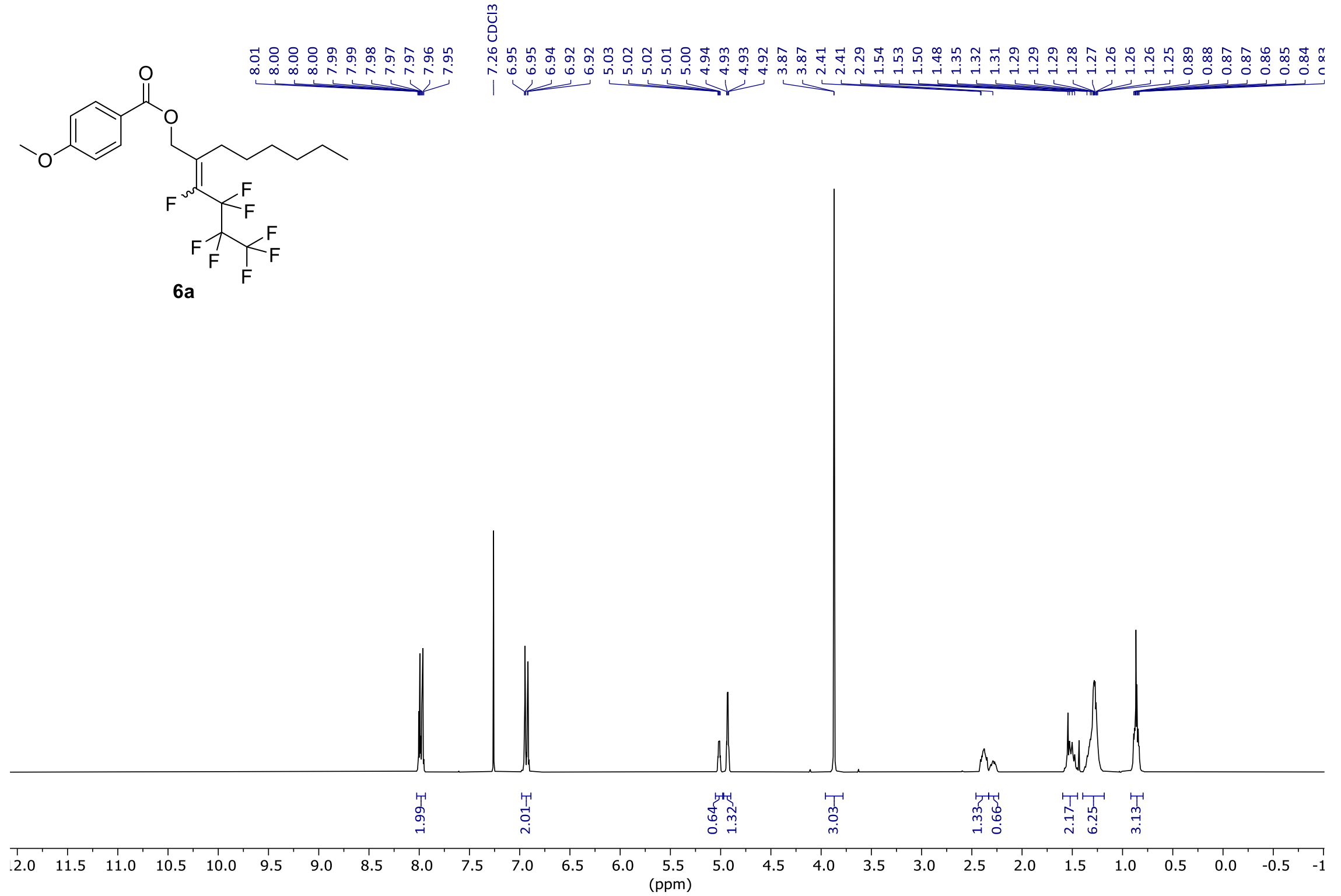


$13 \mathrm{C}\{1 \mathrm{H}\} \mathrm{NMR}, \mathrm{CDCl} 3,151 \mathrm{MHz}$

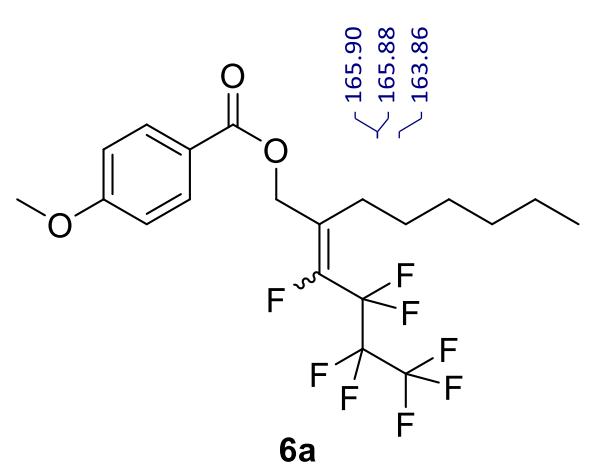

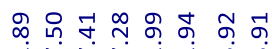

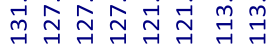

각
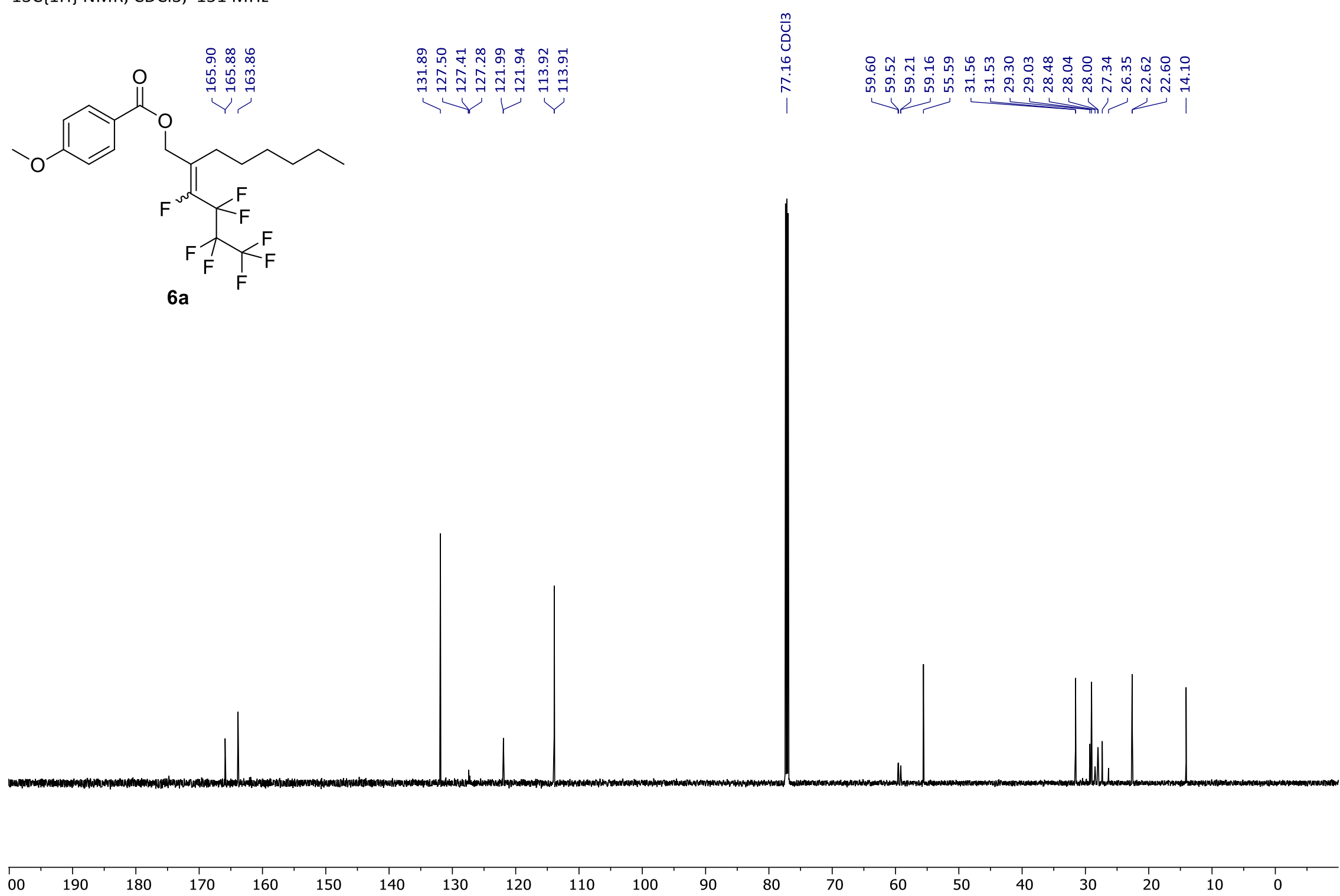
19F NMR, CDCl3, $282 \mathrm{MHz}$

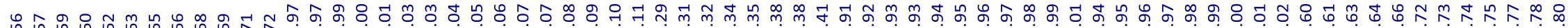

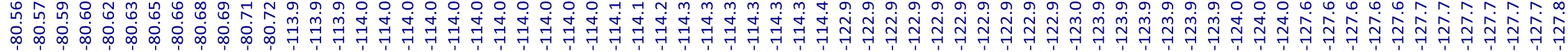
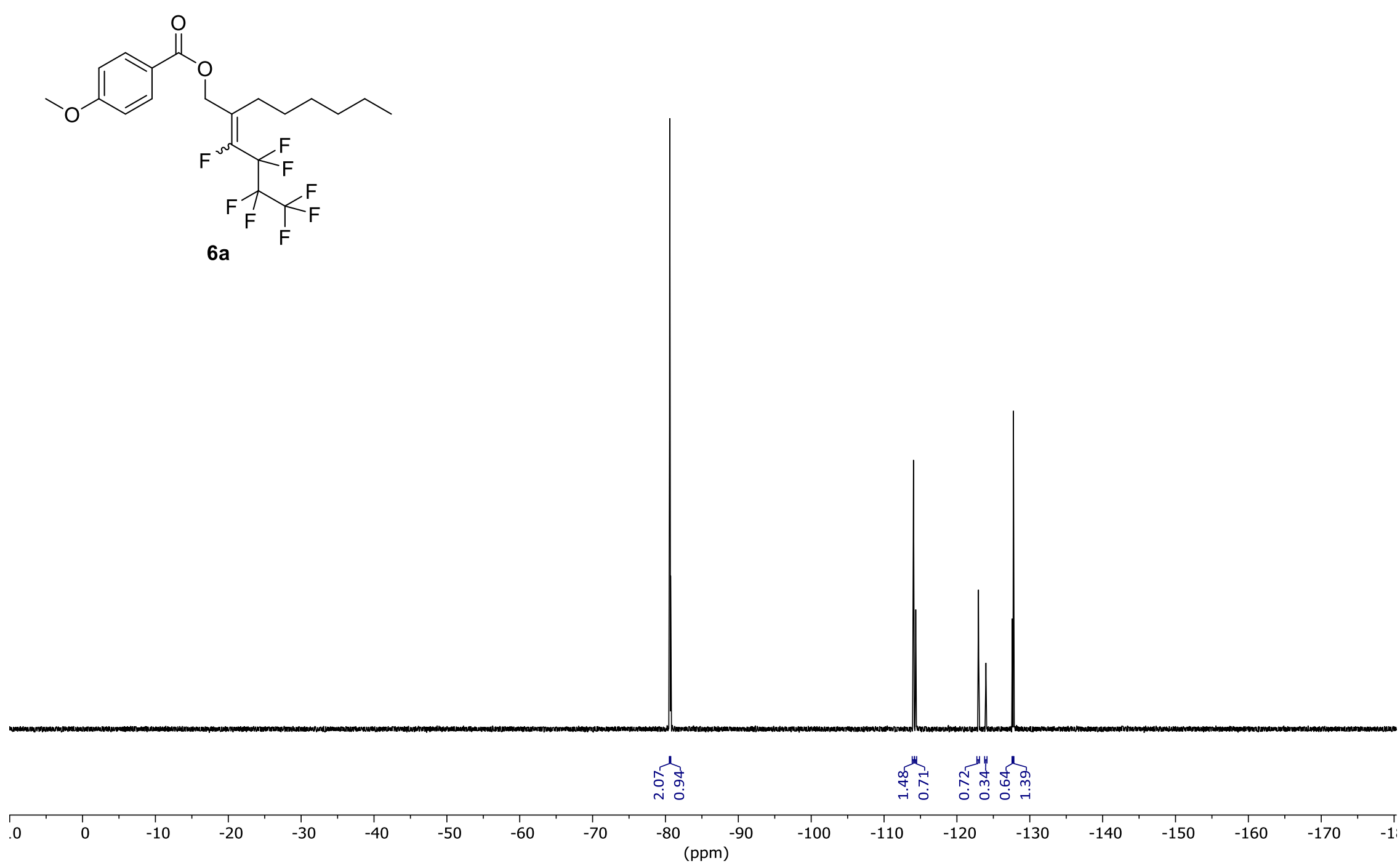
$1 \mathrm{H} \mathrm{NMR,} \mathrm{CDCl} 3,300 \mathrm{MHz}$

บั

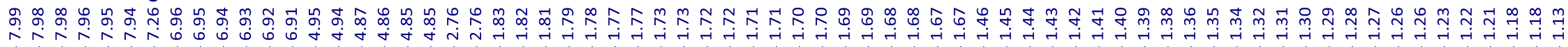

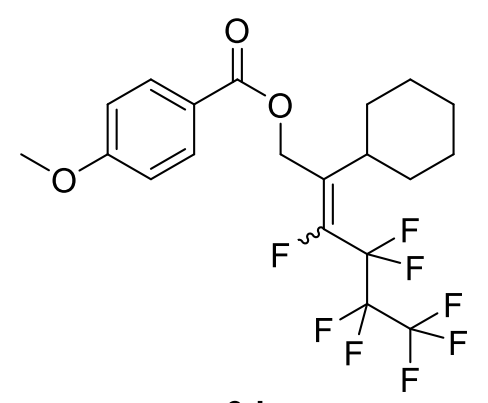

$6 d$

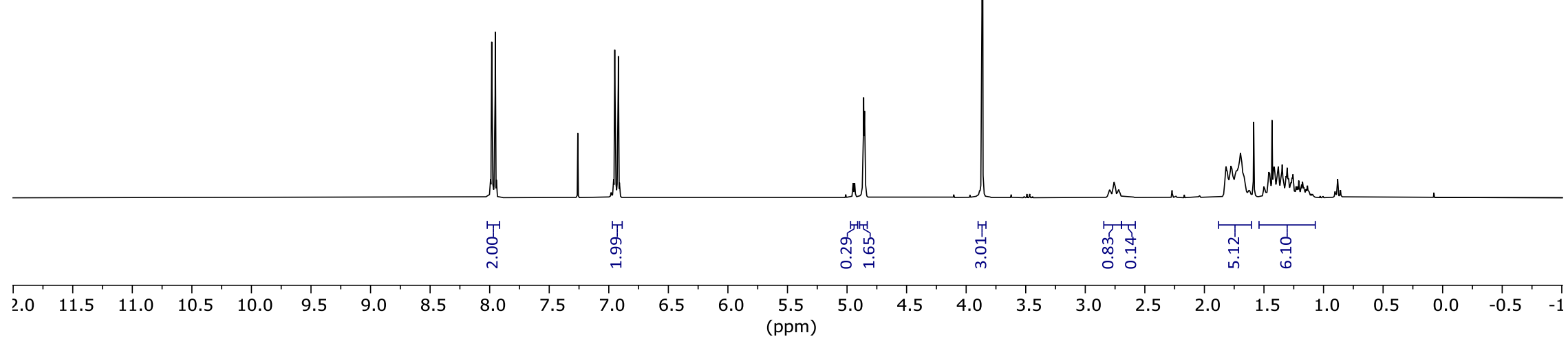


$13 \mathrm{C}\{1 \mathrm{H}\} \mathrm{NMR}, \mathrm{CDCl} 3,151 \mathrm{MHz}$

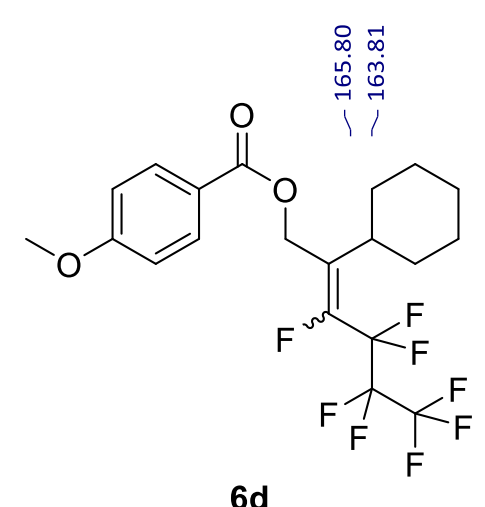

$\stackrel{m}{\mathrm{U}}$

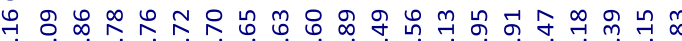

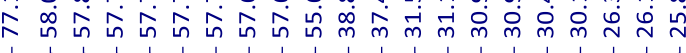

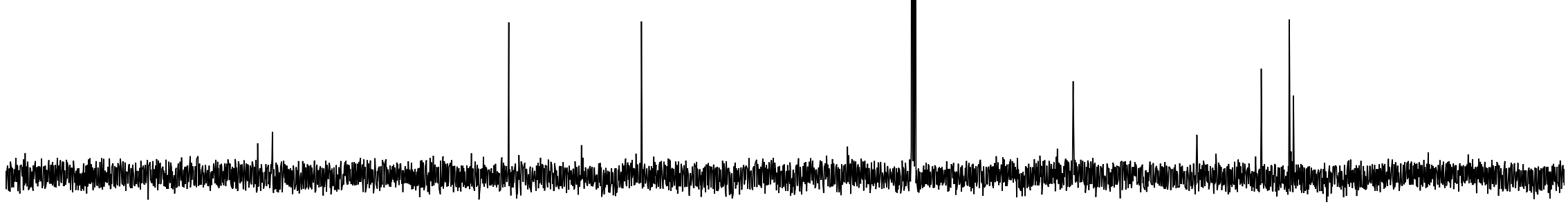

$190 \quad 180$

160

140

120

110

(ppm)

80

$60 \quad 50$

$\begin{array}{llllll}40 & 30 & 20 & 10 & 0 & -1\end{array}$ 
19F NMR, CDCl3, $282 \mathrm{MHz}$

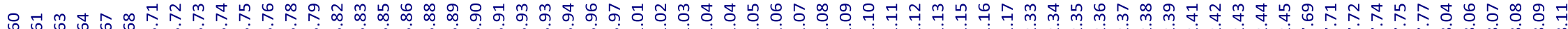

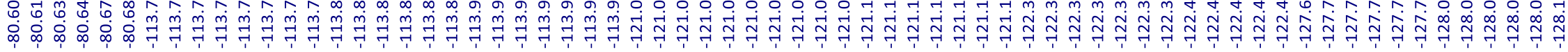

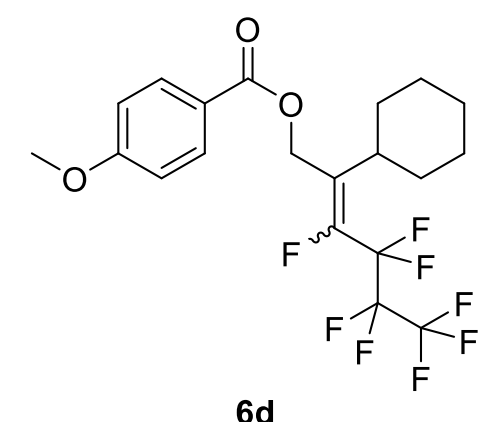

6d

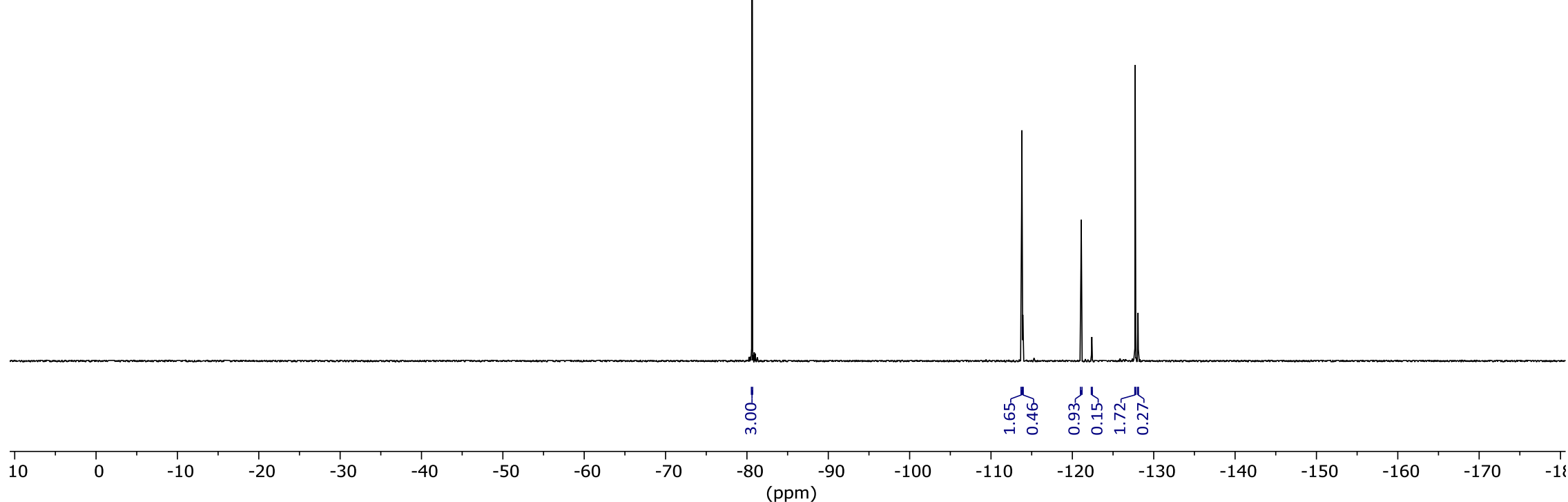

